\title{
Combining Molecular Spintronics with Electron Paramagnetic Resonance: The Path Towards Single-Molecule Pulsed Spin Spectroscopy
}

\author{
Michael Slota ${ }^{1}$ (D) Lapo Bogani $^{1}$ (D)
}

Received: 6 July 2020 / Revised: 12 October 2020 / Accepted: 21 October 2020 /

Published online: 18 November 2020

(c) The Author(s) 2020

\begin{abstract}
We provide a perspective on how single-molecule magnets can offer a platform to combine quantum transport and paramagnetic spectroscopy, so as to deliver timeresolved electron paramagnetic resonance at the single-molecule level. To this aim, we first review the main principles and recent developments of molecular spintronics, together with the possibilities and limitations offered by current approaches, where interactions between leads and single-molecule magnets are important. We then review progress on the electron quantum coherence on devices based on molecular magnets, and the pulse sequences and techniques necessary for their characterization, which might find implementation at the single-molecule level. Finally, we highlight how some of the concepts can also be implemented by including all elements into a single molecule and we propose an analogy between donor-acceptor triads, where a spin center is sandwiched between a donor and an acceptor, and quantum transport systems. We eventually discuss the possibility of probing spin coherence during or immediately after the passage of an electron transfer, based on examples of transient electron paramagnetic resonance spectroscopy on molecular materials.
\end{abstract}

\section{Introduction}

In the last decades, the manufacturing of microelectronic structures on a length scale of tens of nanometers has enabled potent and highly efficient electronic devices that facilitate our daily life. Since miniaturization of pure silicon nanostructures

Dedicated to Prof. Dante Gatteschi, with friendship, and a hug.

Lapo Bogani

lapo.bogani@materials.ox.ac.uk

Michael Slota

michael.slota@materials.ox.ac.uk

1 Department of Materials, Universty of Oxford, 16 Parks Rd, Oxford OX1 3PH, UK 
is approaching the end of the road, scientists and engineers are working together towards alternative materials leading to a further reduction of manufacturing processes [1]. To demand the necessities of the future, the development of transistors on the single-molecule level is getting more and more important, as it represents the ultimate form of miniaturization. At such a level, the electronic behaviour of a quantum device is strongly affected by the magnetic property of the electron, the electron spin, leading to interesting quantum phenomena and physics, which differ from those of bulk systems [2].

The combination of molecular electronics with single-molecule magnets (SMMs) led to the innovative field of molecular spintronics [3], which enabled transistors $[4,5]$, switches [6] and electronic read-out of nuclear spins [7] on single-molecule length scales. SMMs are molecular structures with a metallic core, which act as a tiny magnet below a certain blocking temperature $[8,9]$. Generally surrounded by organic ligands, these coordination complexes can be functionalized and deposited on conducting surfaces such as gold, carbon nanotubes or graphene [2]. Therefore, SMMs are the components of choice for the investigation of novel molecular spintronic devices and observations of yet unknown spintronic phenomena at cryogenic temperatures. Although progress has been made in the fabrication of such devices, quantum coherence of integrated SMMs has not been resolved so far. Exploiting quantum coherence of SMMs could lead to novel controllable quantum logic elements [10], especially if obtained at the single-molecule level. Direct access to quantum coherence is possible by electron paramagnetic resonance (EPR) spectroscopy [11]. The challenging task of combining EPR with molecular spintronics could open up a new horizon for spin manipulation and quantum computing.

In this work, we address the possibilities that SMMs offer for molecular spintronics and discuss a possible way combining EPR with spintronics. Due to the molecular structure of SMMs, the local environment plays an important role. Thus, we begin with an overview on the properties of SMMs, making them interesting objects for molecular spintronics. The second chapter deals with current state-ofthe-art developments in molecular spintronics. We will address current issues and show first attempts of nuclear magnetic resonance spectroscopy on SMMs in nanodevices. Furthermore, we will see how the local surroundings of the SMMs perturbs their properties. To combine EPR with molecular spintronics, we will propose to attach SMMs on donor-acceptor molecules, in which currents can be generated via photoexcitation. Thus, the last chapter focuses on quantum coherence properties and excited state spin dynamics investigated by EPR.

\section{Magnetic Nanomaterials}

Integrating single-molecule magnets (SMMs) and other nanomagnetic materials as key ingredients into new environments, such as molecular cages [12] or nanoelectronic devices [3], had revealed fascinating quantum phenomena [2, 8]. For a better understanding of the observations discussed in the following chapters, we will give an overview about the basic properties and appealing features of SMMs, which researchers are about to exploit for molecular spintronics and quantum computation. 
We will discuss how bulk properties can be modeled and how they become affected by environmental effects.

\subsection{Basics of Molecular Magnetism}

\subsubsection{Single-Molecule Magnets}

Single-molecule magnets are a special kind of magnetic material, where the magnetic hysteresis is intrinsically associated with single molecules [8] and, in contrast to ferromagnets, long-range cooperative exchange interactions are not required. SMMs represent the ultimate miniaturization of magnetic materials, as every single molecule behaves as a tiny magnet, yielding a fruitful ground for molecular spintronics [3] and quantum computation [10]. Like superparamagnets, SMMs retain their magnetization for long periods of time below a certain blocking temperature, $T_{\mathrm{B}}$, which can exceed many hours at sufficiently low temperatures [13]. Usually, $T_{\mathrm{B}}$ is defined as the temperature where the magnetization is retained for at least $100 \mathrm{~s}$ after removal of an external magnetic field. Above $T_{\mathrm{B}}$, thermal fluctuations drive the system into a paramagnetic equilibrium state. The thermal relaxation time $\tau$, which corresponds to the characteristic time for a spin-flip (Néel relaxation), can be generally expressed as [13]

$$
\tau=\tau_{0} \exp \left(\frac{U_{\text {eff }}}{k_{\mathrm{B}} T}\right),
$$

where $\tau_{0}$ denotes the inverse spin-flip attempt frequency (typically $10^{-6}-10^{-12} \mathrm{~s}$ ), $U_{\text {eff }}$ the magnetic anisotropy barrier, $k_{\mathrm{B}}$ the Boltzmann constant and $T$ the system temperature.

The unique feature of SMMs is definitely their monodispersity. In contrast to superparamagnetic nanoparticles, every spin center and thus every molecule acts as a magnet by itself. Fabricated nanoparticles show a distribution of sizes, which is why stochastic behavior is observed and many efforts are being undertaken to narrow down the distribution $[14,15]$. Furthermore, as SMMs are constructed via bottom-up approaches, the composition and structure are known and the atomic positions are well defined in geometry, which is a huge advantage for the integration into nanoelectronic devices and the interpretation of the phenomena.

The first SMM, a $\mathrm{Mn}_{12} \mathrm{Ac}$-complex $\left[\mathrm{Mn}_{12} \mathrm{O}_{12}(\mathrm{OAc})_{16}\left(\mathrm{H}_{2} \mathrm{O}\right)_{4}\right]$ with a blocking temperature of $3.1 \mathrm{~K}$, was discovered in the early $90 \mathrm{~s}[16,22]$. It consists of a magnetic center that is formed by several exchange-coupled metal ions, which are coordinated by organic ligands. In this case, a set of eight $\mathrm{Mn}^{\mathrm{IV}}$-ions with a spin of $s=2$ show antiferromagnetic coupling with four $\mathrm{Mn}^{\mathrm{III}}$-ions with $s=3 / 2$. The result is a large spin state of $S=10$ creating a strong uniaxial magnetic anisotropy. The presence of local distortions in the ligands can create sizeable transverse anisotropy terms. Carboxyl groups $\left(\mathrm{RCOO}^{-}\right)$and oxygen ions $\left(\mathrm{O}^{2-}\right)$ were employed as linkers. Physicists and chemists worked together to model the behaviour and created an abundant family of new molecular magnetic materials (see Fig. 1). 
(a)

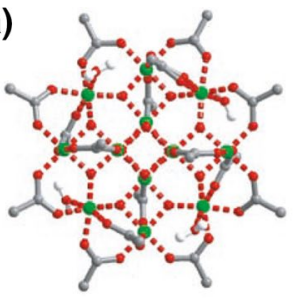

(c)

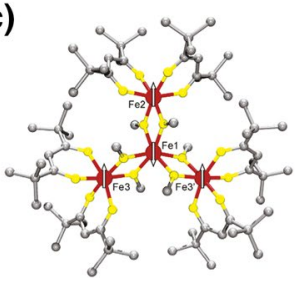

(b)

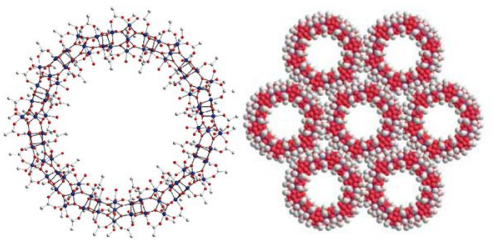

(d)

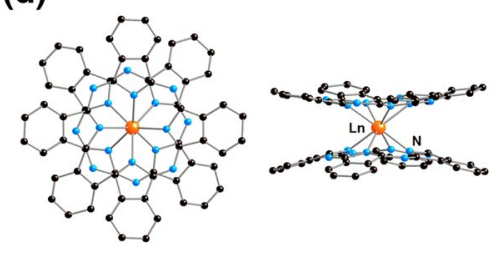

(e)

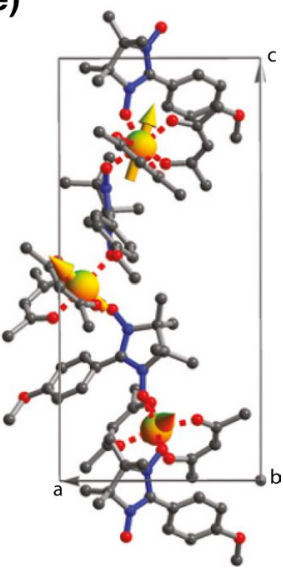

Fig. 1 Selected structures of molecular magnets. a The archetypal $\mathrm{Mn}_{12} \mathrm{Ac}$ cluster with $S=10$ (from Ref. $[3,16]$ ). b $\mathrm{Mn}_{84}$ torus and hexagonal packing in tube structure (from Refs. [17]). c $\mathrm{Fe}_{4}(\mathrm{OMe})_{6}$ $(\mathrm{dpm})_{6}$-cluster $(\mathrm{Hdpm}=$ dipivaloylmethane $)$ as precursor for a series of $\mathrm{Fe}_{4}$-based SMMs, with exchange-coupled iron ions ( $s=5 / 2$ each) yielding an $S=5$ ground state (from Ref. [18]). d The archetypal lanthanide-based SMM [LnPc $] \quad(\mathrm{Ln}=\mathrm{Tb}$ or $\mathrm{Dy}, \mathrm{Pc}=$ phthalocyanine $)$ with only one metal center (from Refs. [19, 20]). Due to strong spin-orbit coupling of lanthanide ions, no exchange-coupling is necessary for SMM behavior. This sub-class of SMMs is also referred to as Single-Ion-Magnets (SIMs). e Assembly of $\mathrm{Co}(\mathrm{hfac})_{2}$ (NIT-PhOMe) molecules to a single-chain magnet (SCM) as a magnetic nanowire (hfac $=$ hexafluoroacetylacetonate, NIT-PhOMe $=$ 2-(4-methoxyphenyl)-4,4,5,5-tetramethyl-1,3-dioxidoimidazol-1-ium) (from Ref. [21]). Reprinted with permission from Springer Nature, Wiley-VCH and Copyright $(2003,2006,2013)$ by the American Chemical Society

Via functionalization, the properties of SMMs can be tailored to display desired features. By substituting parts of the organic shell with surface-binding groups, SMMs can be deposited on conducting surfaces like gold or carbon nanotubes [3]. Moreover, covalent binding to defective sites or reactive groups are possible. This remarkable property enables direct integration into nanoelectronic circuits, as further discussed in Sect. 3. Furthermore, magnetic field and temperature are not the only external stimuli that manipulate the behavior of SMMs. By inserting the appropriate ligands, photons are able to control the spin state of SMMs [23, 24], which could be exploited for quantum logic operations. Further stimuli imply the use of pressure $[25,26]$ or electric fields $[27,28]$. The control of magnetic properties via light is further reviewed in Sect. 4.4.

We note that SMMs do also have drawbacks compared to nanoparticles, which originate from their molecular nature. Being a promising candidate for molecular spintronics [3], the interaction between electrodes and the surrounding ligands, however, often yields to a loss of SMM behavior [29, 30]. Surfaces may lead to a reduction of the metal ions, deteriorating the magnetic properties. Moreover, for integration into molecular spintronic devices, an SMM may need to be functionalized [31] and be able to survive the deposition process [32,33], such as a high deposition temperature. Taken these conditions together, current research not only focuses on reaching large anisotropy barriers and high hysteresis temperatures, but also on the construction of coordination complexes that are chemically stable, redox-stable, and 
overall minimally altered by immediate surroundings such as surfaces, or manipulated during the fabrication process. Moreover, these molecules need to be thermally stable, depending on the fabrication technique, and not reactive with surfaces or electrodes. At the same time, control over the molecular orientation in a device is of crucial importance, so that high yields and reproducible results are guaranteed. Fulfilling these criteria is a challenging task, and often the possible molecules are structurally complex and show a low symmetry, making the investigation of their properties even more challenging. Therefore, only a fraction of the plethora of SMM complexes [34] can eventually be integrated into spintronic devices. Promising candidates, amongst others, are $\mathrm{TbPc}_{2}$-based [35] and propeller-shaped $\mathrm{Fe} 4 / \mathrm{Fe}_{3} \mathrm{M}$-based SMMs [36]. These effects are discussed more in detail in Sect. 3.

\subsubsection{Slow Relaxation and Quantum Tunneling of Magnetization}

The magnetic blocking originates from the slow relaxation of magnetization, a phenomenon first observed on SMMs in 1991 [37]. The fascinating thing is that both classical and quantum physics play a role, even at the same time. The energy diagram (double-well potential) of the zero-field splitting is shown in Fig. 2 for $\mathrm{Mn}_{12}$ Ac. The ground multiplet with $S=10$ splits into $2 S+1$ states. At sufficiently low temperatures, only the lowest lying state is occupied. Depending on the magnetic field, the system can be polarized in $M_{S}=+S$ or $M_{S}=-S$. The magnetization can then be inverted via three pathways.

Firstly, it can be inverted thermally. When increasing the temperature, excited states are getting populated obeying a Boltzmann distribution. Phonon-induced transitions with $\Delta M_{\mathrm{S}}= \pm 1$ lead to population of states on the counter side of the energy barrier. If the thermal energy is larger than the anisotropy barrier $U_{\text {eff }}$, the thermal fluctuations dominate the system and the SMM behaves as an usual paramagnet.

(a)

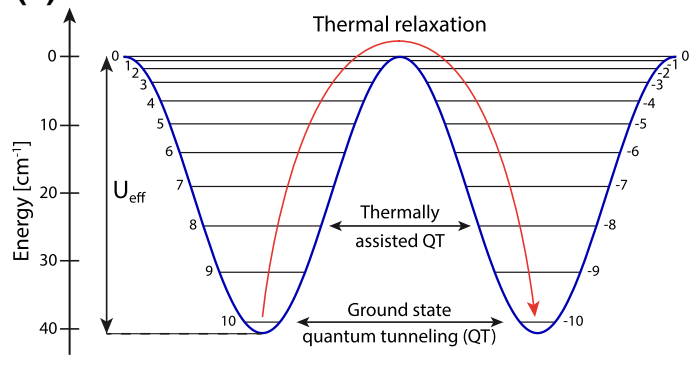

(b)

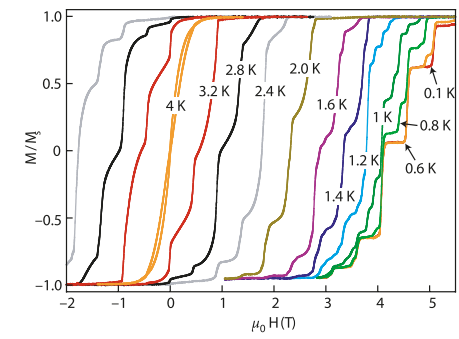

Fig. 2 Slow relaxation of magnetization. a Relaxation mechanisms in $\mathrm{Mn}_{12} \mathrm{Ac}$-SMMs. At very low temperatures, only the lowest lying state is occupied and magnetic reversal is thermally blocked. b Magnetic hysteresis of $\mathrm{Mn}_{12} \mathrm{Ac}-\mathrm{SMMs}$. The characteristic steps in the hysteresis loop appear when two energy levels are in resonance when sweeping the magnetic field. Due to the Zeeman effect, a magnetic field shifts the states of both sides in opposite directions). In resonance, quantum tunneling of magnetization is possible. The appearance of hysteresis is strongly dependent on the field sweep rate. Picture adapted from Ref. [3]. Reprinted with permission from Springer Nature 
Second, inversion takes place via quantum tunneling of magnetization, as first observed by Friedman et al. in [38]. In fact, the energy diagram shown in Fig. 2 is only partially correct. Due to the occurrence of transverse anisotropy $E$, resulting from local distortions, the $M_{S}$ states are not pure states but have admixtures of different states. For $\mathrm{Mn}_{12} \mathrm{Ac}$ these are mixtures with $\Delta M_{\mathrm{S}}= \pm 4$. Thus, the $\mid \pm 10>$ states are mixed, with a small degeneracy lifting, which creates the barrier for quantum tunneling. The steps in magnetic hysteresis curves of SMMs are a typical hint for quantum tunneling of magnetization (Fig. 2b).

As a third possibility, thermally assisted quantum tunneling can also take place. This behavior shows clearly that in molecular magnetism classical and quantum behavior occur in parallel, which makes SMMs fascinating objects for scientists.

\subsubsection{Requirements of SMMs}

To behave as an SMM, the anisotropy barrier must be sufficiently large. In the simplest approximation for transition metal ions with total integer spin, it is given by $U_{\text {eff }}=D S^{2}$, where $D$ is the axial anisotropy parameter $[8,19]$ (see next section). The $D$ value depends on the electrostatic interactions between the magnetic center and the organic ligands, and the anisotropic inter-centre interactions (e.g. dipole-dipole coupling). For negative values, spin states with the largest projection along the anisotropy axis are preferred, resulting in a strong easy-axis anisotropy.

To obtain larger anisotropy barriers and thus higher blocking temperatures, scientists tried to maximize the spin by huge exchange-coupled clusters. However, it has been found that a large spin state usually comes together with a small axial anisotropy parameter [19]. The $\mathrm{Mn}_{19}$-cluster with a record spin of $83 / 2$ only shows $U_{\text {eff }}=4 \mathrm{~cm}^{-1}$ [39], while $\mathrm{Mn}_{12}$ Ac with $S=10$ shows $U_{\text {eff }}=51 \mathrm{~cm}^{-1}[16,22]$. This is due to the fact that in such large clusters, the single anisotropy terms of the ions mutually cancel out [19]. Therefore, scientists started to find ways to increase singleion anisotropy.

Lanthanide ions show a strong single-ion anisotropy due to their unquenched orbital angular momentum. Ishikawa et al. where the first to show a single-molecule magnet with only one central lanthanide ion [20] (Fig. 1d). Many lanthanide-based SMMs followed [19, 40-43], and even 3d single-ion magnets have been made possible [44, 45]. For a long time, the highest blocking temperature achieved with lanthanides was $14 \mathrm{~K}$ [46], which is still far away from room temperature. In 2017, the first SMM with a magnetic hysteresis at $60 \mathrm{~K}$ was reported [47], and in 2018 even $80 \mathrm{~K}$ were achieved [48]. Anisotropy barriers are now exceeding $2000 \mathrm{~K}$ [48, 49]. These recent milestones are giant steps towards potential room temperature operations, showing there is still ample room for improvement of the structure [50]. Nevertheless, despite the intense research, the largest drawback of SMMs remains their low blocking temperatures. 


\subsubsection{Theoretical Description of SMMs}

The fascinating properties of SMMs are a result of the different magnetic effects, which are responsible for the zero-field splitting of the system. It can be described by the Spin Hamiltonian model, which is a summation over the single Hamiltonians $[8,51]$ given by

$$
\mathcal{H}=\mathcal{H}_{\mathrm{Z}}+\mathcal{H}_{\mathrm{CF}}+\mathcal{H}_{\mathrm{JJ}}+\mathcal{H}_{\mathrm{HF}}+\mathcal{H}_{\mathrm{NZ}}+\mathcal{H}_{\mathrm{NQ}}
$$

with

$$
\begin{array}{cc}
\mathcal{H}_{\mathrm{Z}}=\mu_{\mathrm{B}} \mathbf{B} \cdot \mathbf{g} \cdot \mathbf{S} & \text { (Zeeman effect) } \\
\mathcal{H}_{\mathrm{CF}}=\sum_{k \geq 2,|q| \leq k} B_{q}^{k} \hat{\mathbf{O}}_{q}^{k} & \text { (Crystal-field interaction) } \\
\mathcal{H}_{\mathrm{JJ}}=\sum_{j \neq i} \mathbf{S}_{\mathbf{i}} \cdot \mathbf{J}_{\mathbf{i j}} \cdot \mathbf{S}_{\mathbf{j}} & \text { (Exchange coupling) } \\
\mathcal{H}_{\mathrm{HF}}=\sum_{i} \mathbf{S} \cdot \mathbf{A}_{\mathbf{i}} \cdot \mathbf{I}_{\mathbf{i}} & \text { (Hyperfine coupling) } \\
\mathcal{H}_{\mathrm{NZ}}=\mu_{n} g_{n} \mathbf{B} \cdot \mathbf{I} & \text { (Nuclear Zeeman effect) } \\
\mathcal{H}_{\mathrm{NQ}}=\mathbf{I} \cdot \mathbf{P} \cdot \mathbf{I} \quad \text { (Nuclear quadrupole interaction) }
\end{array}
$$

The Zeeman term describes the interactions of a spin $\mathbf{S}$ with a magnetic field $\mathbf{B}$, where $\mathbf{g}$ denotes the $g$-tensor of the magnetic center and $\mu_{B}$ the Bohr magneton. The crystal-field (CF) term is a result of the electrostatic Coulomb interactions of the electrons of unfilled orbitals with the electrostatic potential of the ligands. Since the anisotropy barrier height depends on the crystal-field parameters, large values are the most important ingredient for SMM behavior. However, the CF parameters strongly depend on the environment of the spin center, which is the crucial point why many SMMs lose magnetic properties when deposited on surfaces. Furthermore, it has been shown that tiny changes in the surrounding or in the magnetic center can have a huge impact on magnetic properties even totally inverting anisotropy axes $[52,53]$ (see Fig. 3). The second-order approximation of the CF Hamiltonian can be simplified giving [8]

$$
\mathcal{H}_{\mathrm{CF}}=D S_{z}^{2}+E\left(S_{x}^{2}-S_{y}^{2}\right)
$$

where $D=3 B_{0}^{2}$ is commonly referred to as the axial anisotropy parameter, and $E=B_{2}^{2}$ as the transverse anisotropy term. Higher order terms have minor influences on transition metal ions [8, 54], but considerable ones on lanthanides [51, 55]. In $\mathrm{Mn}_{12} \mathrm{Ac}$-clusters, these are responsible for state-mixing and thus quantum tunneling 
(a)

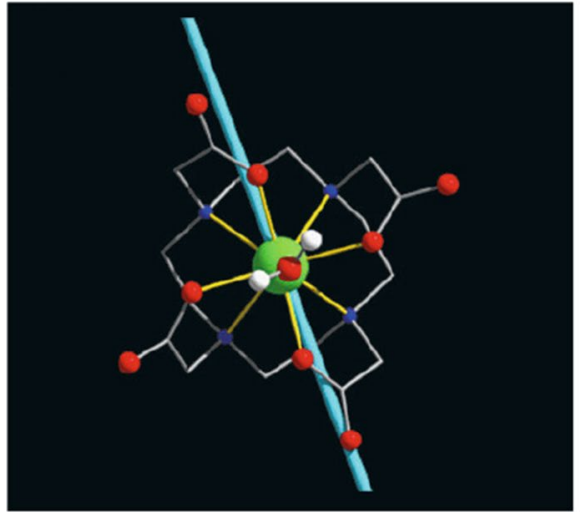

(b)

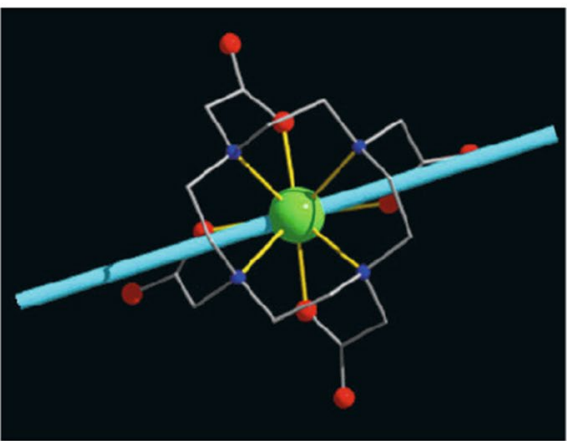

Fig. 3 Calculations of environmental effects on magnetic properties of a LnDOTA-complex. The preferred orientation of magnetization is denoted by the blue easy-axis, here exemplarily for a LnDOTAcomplex. Removing the central water molecule results in a 90-degree rotation of the easy-axis. Pictures adapted from Ref. [52]. Reprinted with permission from Wiley-VCH

of magnetization [8]. The number and kind of higher order terms strongly depend on the symmetry of the SMM [51].

The exchange-interaction Hamiltonian incorporates the exchange-coupling tensor $\mathbf{J}$, which can be reduced to a sum of isotropic exchange (Heisenberg-type [56]), anisotropic exchange and antisymmetric exchange (Dzyaloshinskii-Moriya interaction $[57,58])$. Usually, the isotropic term $-J_{i j} \mathbf{S}_{\mathbf{i}} \cdot \mathbf{S}_{\mathbf{j}}$ with $J_{i j}=-\operatorname{Tr}\left(\mathbf{J}_{\mathbf{i j}}\right) / 3$ dominates and produces, depending on the sign of $J_{i j}$, either parallel or antiparallel coupling. The topology of the intramolecular couplings in $\mathrm{Mn}_{12}$ Ac produces a total ground spin state $\quad S=10 \quad[16, \quad 22]$. Anisotropic exchange $\mathbf{S}_{\mathbf{i}} \mathbf{D}_{\mathbf{i j}} \mathbf{S}_{\mathbf{j}}$ with $D_{i j}^{\alpha \beta}=\left(J_{i j}^{\alpha \beta}+J_{i j}^{\beta \alpha}\right) / 2-\delta_{\alpha \beta} \operatorname{Tr}\left(\mathbf{J}_{\mathbf{i j}}\right) / 3$ and antisymmetric exchange $\mathbf{D}_{\mathbf{D M}}\left(\mathbf{S}_{\mathbf{i}} \times \mathbf{S}_{\mathbf{j}}\right)$ with $D_{D M}=\left(J_{i j}^{\beta \gamma}+J_{i j}^{\gamma \beta}\right) / 2$, where $\alpha, \beta, \gamma$ represent Cartesian coordinates, are small and can be treated as perturbations [8].

The last term, the electron-nuclei interaction term, can usually be reduced to hyperfine coupling. This interaction is comparably small (splitting in $\mathrm{mK}$ region). The coupling arises from magnetic dipole-dipole interactions between the nuclei $i$ with nuclear spin $\mathbf{I}_{\mathbf{i}}$, described by the tensor $\mathbf{A}_{\mathbf{i}}$. Hyperfine interaction plays an important role in microwave spectroscopy and decoherence mechanisms (see Sects. 3 and 4). Nuclear Zeeman effect (similar to the standard Zeeman effect but with nuclear $g_{n}$ and nuclear magneton $\mu_{n}$ ) and nuclear quadrupole interaction, where $\mathbf{P}$ denotes the quadrupole tensor, may play a role in some cases.

It has to be noted that the interaction with the environment also strongly depends on the nature of the electronic states within the metal ion [51]. Hereby, electron-electron interactions within the ion and spin-orbit coupling (for lanthanide ions usually) play an important role. This leads to a shift in energy and to different interaction patterns described by the Wybourne formalism [51]. In the case of lanthanides, we have to account for spin-orbit coupling and consider the total angular momentum in the theoretical description. A thorough study on how to determine 
crystal-field parameters in a lanthanide compound using a plethora of spectroscopic techniques and magnometry techniques is demonstrated in Ref. [59].

\section{Molecular Spintronics}

\subsection{What is Spintronics? A Brief Historical Overview}

Spintronics, a rather young research area, investigates how the electron spin can be used as an additional degree of freedom in electronic devices. The foundation of the field was laid down by the discovery of the giant magnetoresistance effect (GMR) in 1988 by Fert et al. and Grünberg et al. $[60,61]$. Both groups independently observed a spin-dependent resistance in alternating thin layers of iron and chromium, where iron is ferromagnetic and chromium paramagnetic (see Fig. 4). By varying the thickness of the chromium layers, the ferromagnetic iron layers couple either ferromagnetically or antiferromagnetically due to RKKY interactions [62-64]. The resistance change $\Delta R / R$ between both configurations amounted to $80 \%$, which is explained by the spin nature of electrons (Fig. 4). Because spins pointing antiparallel to the magnetization of the layers are scattered, a ferromagnetically coupled stack can be used as a spin valve. The importance of their discovery was honored by the Nobel Prize in 2007. Nevertheless, the first theoretical model, the two-current model, was developed by Mott in 1936 [65].

The implementation of the GMR in modern technology revolutionized magnetic data storage in hard disc drives [13, 66, 67]. Two ferromagnetic layers with different coercivities are utilized here. The first layer acts as a polarizer, while the second, with smaller coercivity, as an analyser. More advanced developments using tunnel magnetoresistance effects (TMR) made magnetic storage with enormously high density possible, which we use nowadays [68, 69]. In TMR, the chromium layer is replaced by an insulating layer through which electrons can tunnel. In 1975, first developments of TMR devices resulted in a resistance change of $14 \%$ at liquid helium temperature (4.2 K) [70] and even less at room temperature. In the $90 \mathrm{~s}$, further developments were able to increase the value to several percents at room temperature using $\mathrm{Al}_{2} \mathrm{O}_{3}$ as an insulator layer [71] and reaching 70\% [72]. More
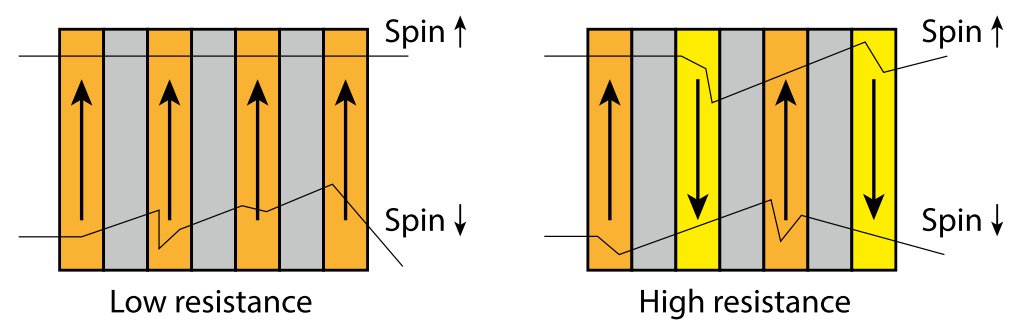

Fig. 4 Principle of the giant magnetoresistance (GMR) effect. The electrons with spins pointing parallel to the ferromagnetic iron layers have a lower resistance than the ones pointing antiparallel. This is due to the fact that antiparallel oriented spins are scattered within the thin ferromagnetic layers. Therefore, a parallel magnetization of the iron layers show a lower resistance than an antiparallel magnetization 
remarkably, by taking $\mathrm{MgO}$ as insulating layer an increase of the relative resistance change to $220 \%$ in $2004[73,74]$ and subsequently to $600 \%(1100 \%$ at $5 \mathrm{~K}$ ) in 2008 [75] were observed, and the trip still goes on.

Such spintronic devices constructed via top-down approaches are massively produced and well established in microelectronic chip devices. To increase the performance of microelectronic devices, the chip industry follows the route of miniaturization. By approaching nanometer scales, quantum properties like quantum tunneling become relevant. Therefore, it is of great importance to investigate the behavior on the single-molecule level to understand the quantum behavior better and to adapt the benefits of quantum effects to improve current technology. Single-molecule magnets [8], in literature also known as molecular nanomagnets, are promising candidates for the design of new spintronic elements on a molecular scale [3]. The resulting new field of molecular spintronics attracted increasing attention in the last 10 years and new effects have been discovered [76-80], opening new particular focus of interests such as spin interfaces ("spinterfaces") [81], and providing potential integration into quantum logic devices [82] and potential applications for quantum information technology $[83,84]$. However, not all of them are yet understood due to experimental limits and remain to be investigated via new techniques.

\subsection{Addressing Single Molecules in Molecular Spintronics}

Addressing single molecules is a challenging task. The experimental setup must both be able to detect single molecules and manipulate them. Thus, some dimensions of the experimental apparatus need to be on the single atom level. Three techniques prove to fulfill these requirements [2], which are schematized in Fig. 5:

1. Attachment of molecules on conducting substrates and investigate spintronic properties using a scanning tunnel microscope (STM) needle as lead.

2. Using break-junctions as leads, where the molecule sits in a nanoscale gap.

3. Using a quantum dot (QD) as host and attach the molecule onto it (double-dot scheme). Here, the electronic quantum dot is the device itself while the molecule provides the spin degree of freedom.

(a)

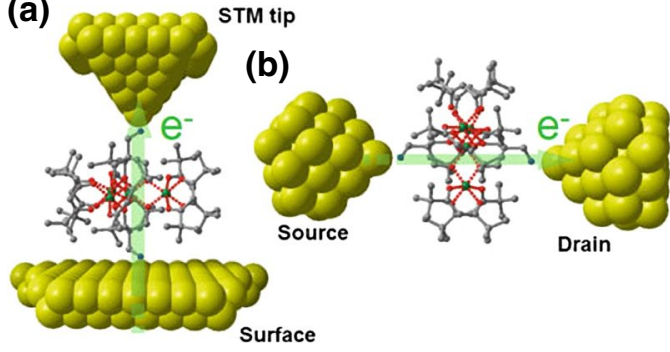

(c)

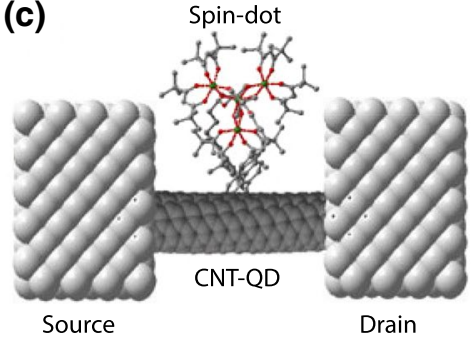

Fig. 5 Investigation techniques in molecular spintronics. a Placing a single molecule on a conducting substrate and use an STM tip as lead. b Placing a single molecule in a break junction. c Double-dot structure, where a single molecule is placed on a contacted quantum dot. Pictures adapted from Refs. $[85,86]$. Reprinted with permission from Springer Nature 
In the following subsections, we will discuss the measurement techniques and its strengths and weaknesses on some state of the art examples. Eventually, we will discuss the drawbacks and draw a possible solution, which adapts techniques commonly used in biology to molecular spintronics.

\subsubsection{Quantum Transport and Basic Measurement Technique}

In metals or semiconductors, electrons form continuous band structures owing to delocalization effects [56]. In molecules, electronic states are generally quantized and electrons are localized (except in a few delocalized groups that form $\pi$ orbitals). Attaching a single-molecule to (metallic) electrodes leads to an interaction between the quantized energy levels of the molecule and the electron reservoir of the electrodes [86]. This is due to an overlap of the electrode wavefunctions with the molecular ones that finally may lead to a change of bulk molecular properties, where basic properties like quantum coherence or magnetic hysteresis of the molecule get lost $[29,30]$.

Due to the quantum nature of molecules, electric currents through molecules behave differently compared to classical systems, as illustrated in Fig. 7. The electronic states of molecules that are connected to electrodes are identical to states of quantum dots, and direct parallels to the conduction behaviors of quantum dots can be drawn. The conduction behavior of such molecular spintronic systems is determined from the stability diagram, where a conductance map is obtained by varying gate and source-drain voltage as shown in Fig. 6. By changing source-drain

(a)

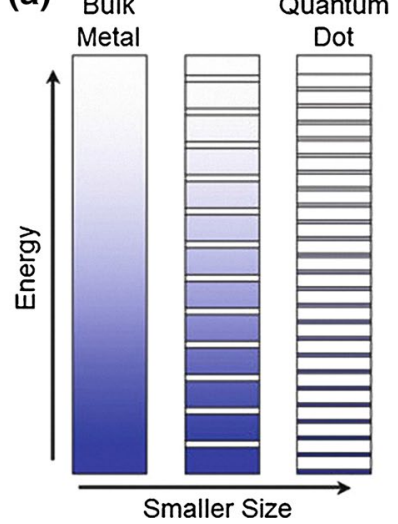

(b)

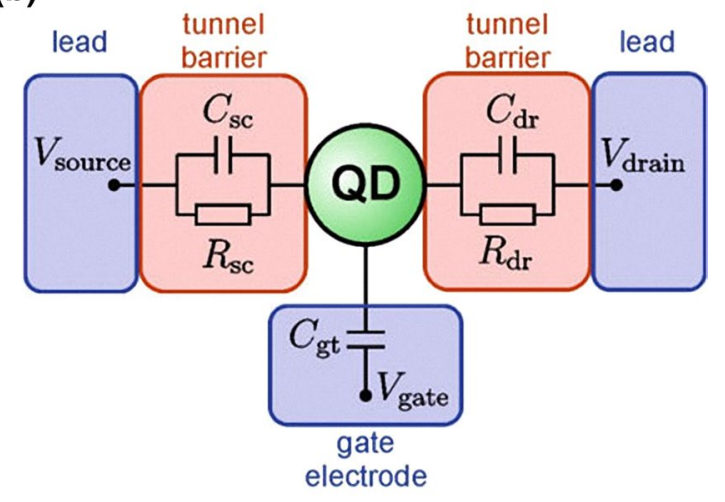

Fig. 6 Size effects on the electronic states, and molecular field-effect transistor (FET). a In a bulk metal, the solution of the Schrödinger equation yields a band structure for the delocalized electrons. The picture represents one band, which can be the conduction band, for instance. When decreasing the size of the metals below typically $10 \mathrm{~nm}$, the electrons are no longer delocalized over the metal and start to form localized states. Then the band model does not hold any longer and such systems can be treated as quantum dots (QDs). b Circuit diagram for a typical quantum dot nanodevice in a field effect transistor scheme. The QD is coupled to leads (source and drain) via tunnel contacts. An additional gate voltage may be directly applied to the QD which shifts the energy levels within the QD. Picture adapted from Ref. [86]. Reprinted with permission from Springer Nature 
(a)

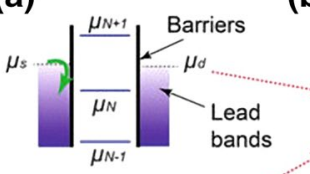

(b)
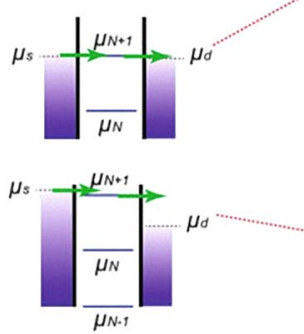

(c)

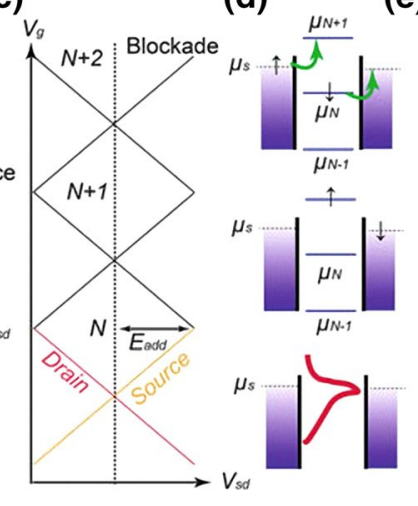

(e)

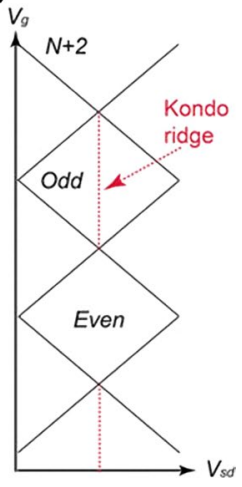

Fig. 7 Electric spectroscopy on quantum dots. a The schematics show the energy level diagram of a quantum dot between two leads, separated by tunnel barriers. Electron transport via tunneling is only possible, when either the highest occupied states of the electrodes are in line with an energy state of the QD [(second figure in (a)] or when both the potential of the drain electrode is lower than of the source and an available QD state lies in between both potentials [third figure in (a)]. When sweeping the source-drain voltage $V_{\text {sd }}$, peaks in the conductance are observed when additional transport channels are getting opened. In the ground state [(first figure in (a)], the numbers of electrons on the QD is fixed and thus no electron transport takes place. $\mathbf{b}$ By adjusting the gate voltage $V_{\mathrm{g}}$, oscillating regimes of conductance can be detected. A similar behavior is observed when $V_{\text {sd }}$ is varied. $\mathbf{c}$ By plotting the conductance against $V_{\mathrm{g}}$ and $V_{\text {sd }}$, so-called Coulomb-diamonds are obtained. d Co-tunneling processes occurring in QD devices with strong hybridization with the leads, which result in a conductance peak without any bias applied, denoted as Kondo ridge in (e). However, this effect vanishes when the QD does not carry a spin, i.e. it carries an even number of electrons. Picture adapted from Ref. [86]. Reprinted with permission from Springer Nature

and gate voltage, regions of higher and lower conductance are obtained, which can be presented as Coulomb-diamonds (Fig. 6b). From this representation, a rich family of parameters are obtained. One gains the energies of adding and removing electrons from the heights of the Coulomb-diamonds, and their widths represent the gate-coupling parameter. The slopes yield the source, drain and gate-coupling parameters [87]. As the stability diagram is affected by external stimuli like magnetic fields, molecular phenomena can be studied by investigating the characteristic Coulomb-diamonds.

\subsubsection{Molecular Spintronics on Surface-Deposited Molecules}

The simplest approach addressing single-molecule spin centers is by surface deposition on a conducting substrate like gold or copper. However, not every SMM is equally suited. The deposition of the archetypal $\mathrm{Mn}_{12}$ clusters has been tried by several scientists [88], but only with very moderate success and inconclusive results [89, 90]. The grafting of $\mathrm{Mn}_{12}$ clusters on a gold surface, for instance, comes along with reduction processes of the manganese ions, which eventually result in a loss of magnetic hysteresis at low temperatures. Albeit molecules themselves could be deposited well according to XAS measurements, XMCD spectroscopy revealed a change in the magnetic nature of the $\mathrm{Mn}_{12}$ cluster. 
Besides interactions with the substrates, the deposition approach suffers from several disadvantages and particular requirements on the molecule [32]. It is advantageous to functionalize molecules with binding ligands to make them stick on a surface. Thiol-terminated aliphatic chains showed to stick well on gold surfaces [91], while phthalocyanines stick on Ni surfaces via non-covalent interactions [92]. Alternatively, adsorption via van der Waals forces can be sufficient on certain surfaces [93].

Since deposition is often performed at high temperatures and ultra-high vacuums, some SMMs may decompose during the process [2, 33]. Therefore, proof of intact molecules is crucial. Thanks to the plethora of SMMs, some indeed resist the

(a)

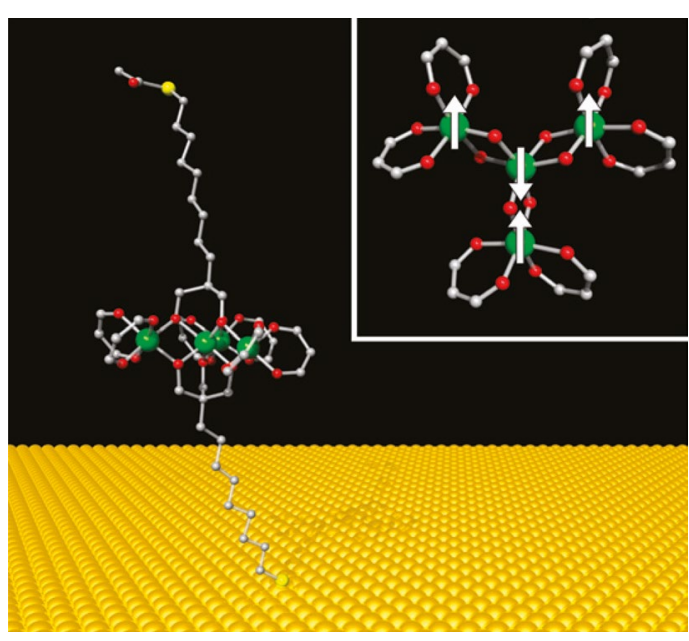

(b)

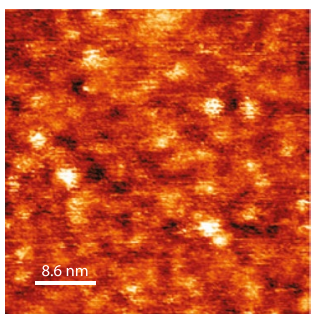

(c)

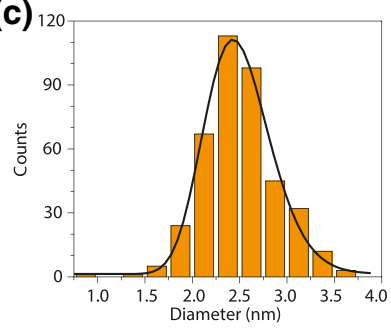

(d)

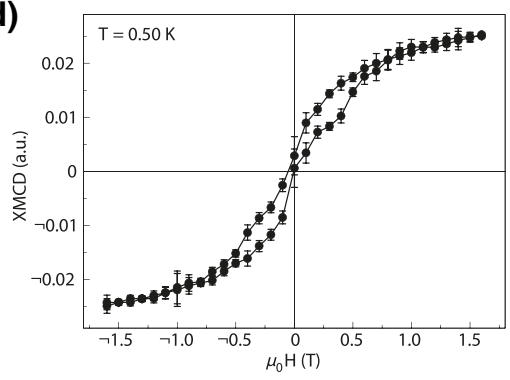

(e)

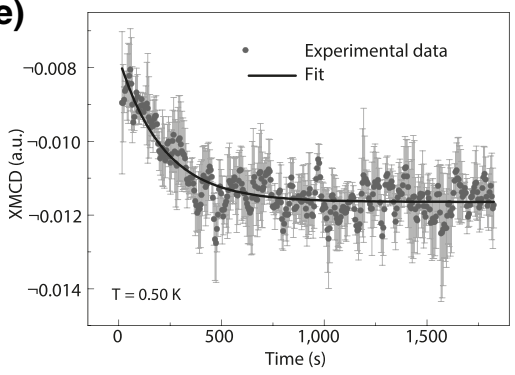

Fig. 8 Monolayer of $\mathrm{Fe}_{4}(\mathrm{~L})_{2}(\mathrm{dpm})_{6}$ on a gold surface. a The schematic picture shows the thiol bridge of the molecule attached to a gold surface. The insight shows the molecular structure of the molecular magnet from the top. The green balls represent the exchange-coupled iron atoms and the arrows denote the orientations of the single spins. b Image from an STM measurement at room temperature, where the spots indicate the positions of the molecules. c Molecule-size distribution, which follows a log-normal distribution law. d Even on the gold surface, the $\mathrm{Fe}_{4}$ molecule maintains hysteresis behavior, as obtained from XMCD measurements. e Time dependence of the dichroic signal at $0.5 \mathrm{~K}$ after magnetizing the sample at $+2 \mathrm{~T}$ and then ramping fast to $-0.25 \mathrm{~T}$. The exponential decay is due to slow relaxation of magnetization below the blocking temperature. Pictures adapted from Ref. [91]. Reprinted with permission from Springer Nature 

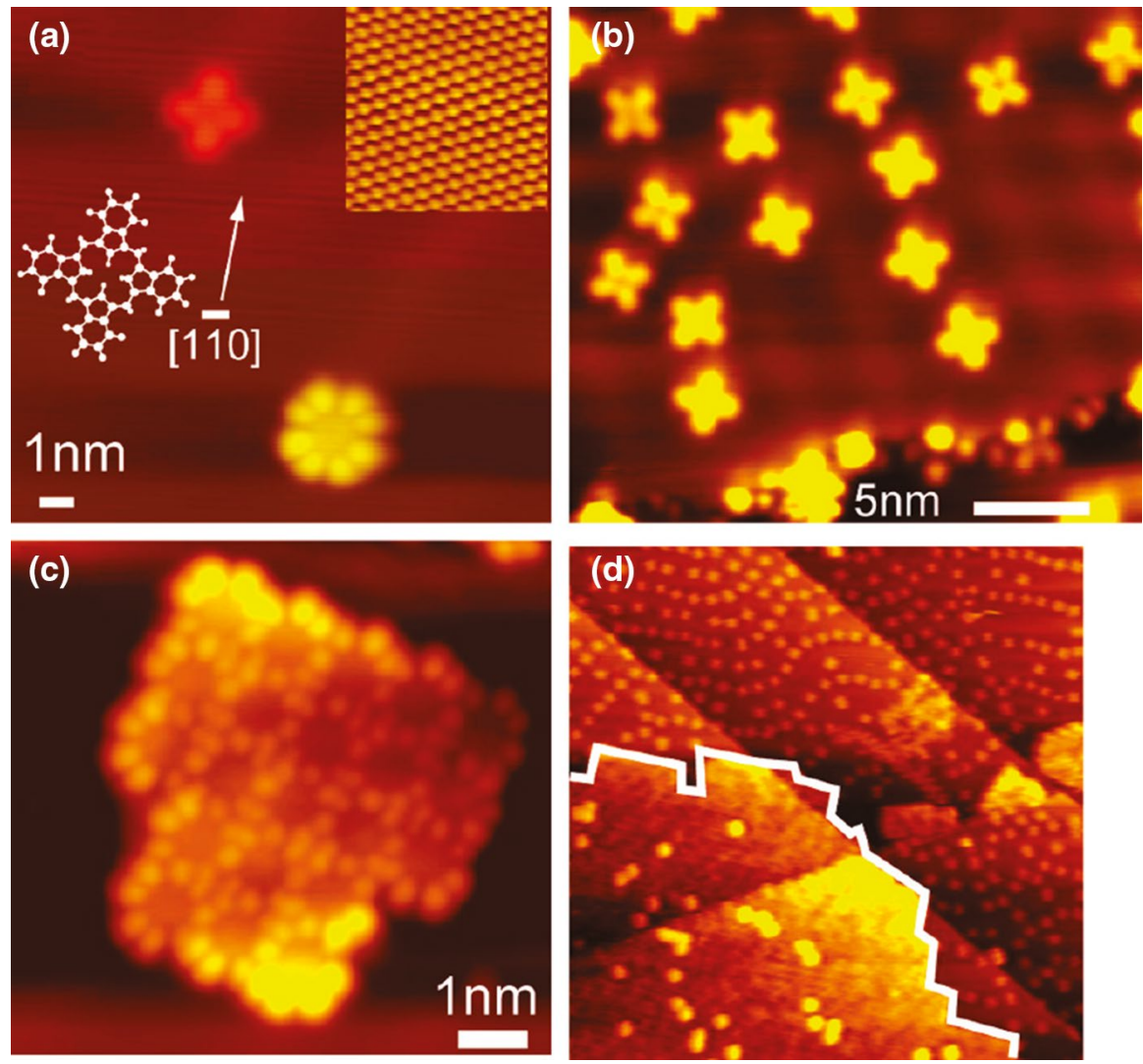

Fig. $9 \mathrm{TbPc}$ and $\mathrm{TbPc}_{2}$ molecules on a Au(111) surface. a The STM images show TbPc species with four lobes and $\mathrm{TbPc}_{2}$ species with eight lobes. The gold surface is shown in the inset. b Randomly oriented $\mathrm{TbPc}$ molecules on gold. c A film of $\mathrm{TbPc}_{2}$ molecules. d Coexistence of both species on the substrate, where the white boundary marks the edge between these two. Picture adapted from Ref. [99]. Reprinted with permission from Copyright (2009) by the American Chemical Society

deposition [2, 32, 94]. Furthermore, not all metal surfaces are equally suited. On the one hand, the substrate has to be reaction stable and should not catalyze chemical reactions. Otherwise, owing to oxidization changes or reorientation of chemical bonds, molecular properties are not preserved. On the other hand, the surface needs to be conductive and easy to handle. A good review about fabrication methods and molecular systems is available in Ref. [95].

The $\mathrm{Fe}_{4}(\mathrm{~L})_{2}(\mathrm{dpm})_{6} \mathrm{SMM}$ [91] and the archetypal lanthanide SMM LnPc 2 [20, 92] have been successfully deposited on gold and nickel surfaces, respectively (Figs. 8 and 9). The terbium derivative $\mathrm{TbPc}_{2}$ is particularly interesting. Müllegger et al. showed that its spin state can be manipulated via radiofrequency pulses as demonstrated in Fig. 10. Since a spin change comes along with different conduction behavior, the radiofrequency technique presents an interesting application for molecular spintronics. Promising studies use conformational switching [96] or different Kondo 
(A)

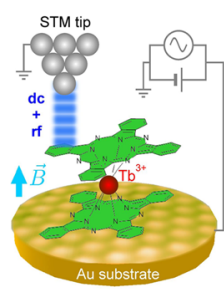

(B)

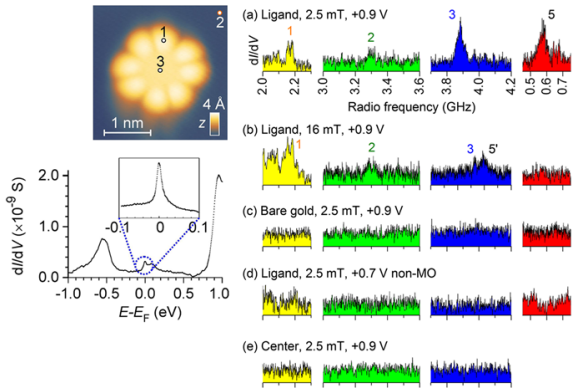

(C)

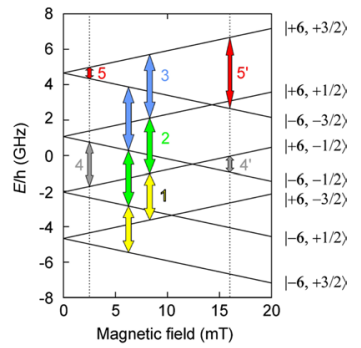

Fig. 10 Radiofrequency STM of $\mathrm{TbPc}_{2}$ on $\mathrm{Au}(111)$. a Setup of the radiofrequency STM. Via an STM tip, tunneling voltage against the substrate can be measured. DC and AC currents are applied simultaneously by different sources. The picture on the right shows an STM image of the $\mathrm{TbPc}_{2}$ molecule at $5 \mathrm{~K}$. Via DC measurements, the characteristic Kondo peak is observed. b By sweeping the AC frequency at $5 \mathrm{~K}$, the conductance shows a peak at certain frequencies. These peaks correspond to spin excitations within the molecule resulting in conductance changes. The measurements conclude that transport through the molecule is directly connected to the molecular spin state of both the electrons and the nuclei, and thus depends on the spin transport channels. By varying the field, the resonance frequencies shift due to the Zeeman effect as denoted in (c). Remarkably, no resonances are observed when the tip is placed centered above the central ion. This is due to vanishing spatial parts of molecular orbitals. For reference, the spectrum of the bare substrate is shown. Pictures adapted from Ref. [100]. Reprinted with permission from Copyright (2014) by the American Physical Society
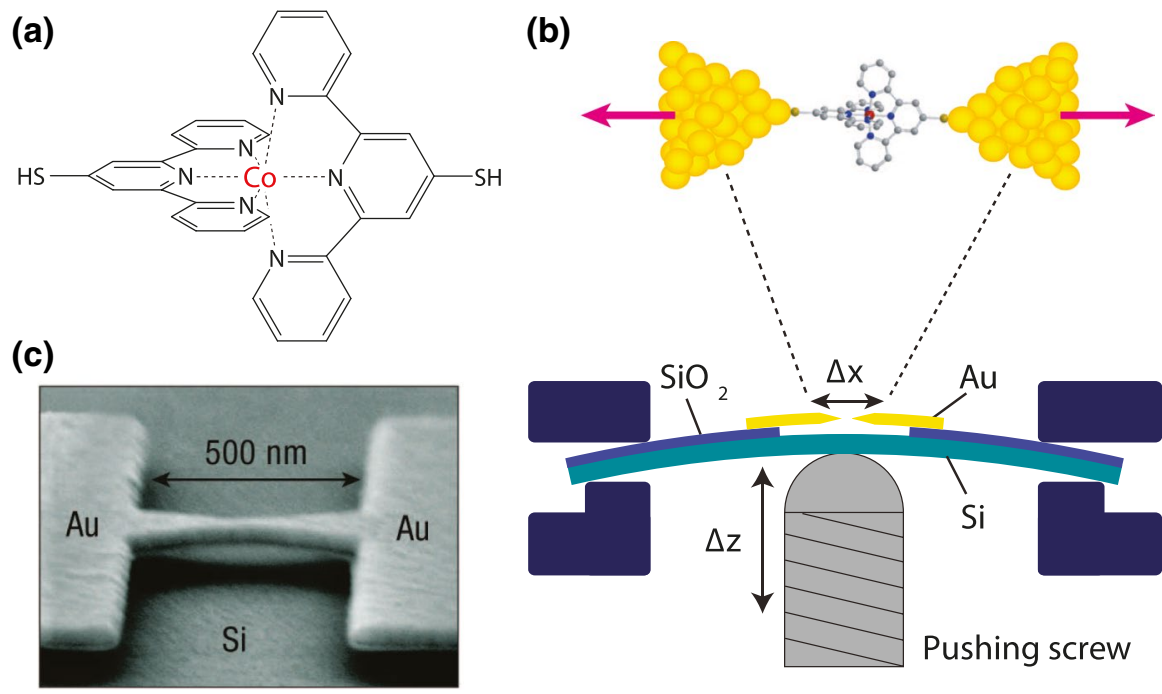

Fig. 11 Break junction example. a Structure of a Co(tpy-SH $)_{2}$ complex. b Illustration of the break junction with the molecule between two gold electrodes. The mechanical axial distortion manipulates the axial anisotropy parameter $D$ and thus the preferred spin state. Therefore, the conduction through the molecule can be controlled. $\mathbf{c}$ SEM picture of the break junction before deposition with molecules. Picture adapted from Ref. [102]. Reprinted with permission from AAAS 
Fig. $12 \mathrm{Mn}_{12}$ Acand $\mathrm{Mn}_{12} \mathrm{Cl}$ characterization via break junctions ( $T=300 \mathrm{mK}$ ). a The variation of the voltage opens different conduction channels which result in steps in the $I-V$-diagram. These steps can be shifted upon applying a gate voltage. b Coulomb-diamonds at different magnetic fields for $\mathrm{Mn}_{12} \mathrm{Ac}$. The green and yellow arrows indicate different transition lines. Only the yellow indicated line shifts upon applying a field. The green indicated line does not shift as it corresponds to an excited state of same spin. c Coulomb-diamonds at different magnetic fields for $\mathrm{Mn}_{12} \mathrm{Cl}$. The yellow arrows mark co-tunneling processes observable for small splittings. At $8 \mathrm{~T}$, a clear Zeeman effect is visible. Pictures adapted from Ref. [101]. Reprinted with permission from Copyright (2006) by the American Chemical Society

signatures upon applying different voltages [97], which affect the hybridization with the surface. DFT analyses are applied to investigate the hybridization [98] and to find further interesting molecules for surface deposition.

\subsubsection{Molecular Spintronics on Break Junctions}

An approach on which researches are more focused at the moment are break junctions. This technique offers the possibility to apply a gate voltage that shifts the energy of the conduction channels of the molecules. In this way, a full characterization of the conduction mechanisms is possible and the technique also enables direct integration of SMMs in field-effect transistors (FETs). An example of a break junction is illustrated in Fig. 11. However, this approach also shares some of the problems of the STM technique. The molecule is again in direct contact with leads which change the electronic structure of the molecule via overlaps of electronic wavefunctions. In addition to that, electrons flow through the metallic spin centers, which results in reduction and oxidization of the molecule. This phenomenon alters the magnetic anisotropy of the molecule.

Studies on $\mathrm{Mn}_{12}$-based break junctions showed that also here magnetic hysteresis vanishes upon grafting to electrodes [101]. Nevertheless, Jo et al. demonstrated the integration into a FET (see Fig. 12). Additional theoretical investigations on the performance have been performed by Heersche et al. [4] enabling direct comparison.

The FET approach offers an elegant way to control the current flow via the gate voltage. Investigations on a vanadium-based dimeric molecule showed that the spin state can be changed from $S=0$ to $S=1 / 2$ by applying a gate voltage. In the $S=1 / 2$ state, a Kondo signature can be measured and the magnetic impurity screens the current [105]. Their studies show that exchange-coupled centers can be used as switches for molecular spintronics. Dimeric systems are even more interesting for quantum computation because they allow the possibility of forming superposition states. Perturbing the interactions between these centers is of current interest in research. Via distinct control of the voltage, the superposition can form either a pseudo-singlet state or a pseudo-triplet state, as illustrated in Fig. 13 [103].

However, to perform quantum logic operations, the quantum coherence time $T_{2}$ must be long (see also Sect. 4). An approach towards measuring quantum coherence times has been shown by Vincent et al. [7] and Thiele et al. [104]. The setup is shown in Fig. 14 and the results are displayed in Fig. 15. The investigations clearly show that pulsed experiments can be performed on break junctions, enabling the possibility of conducting quantum logic manipulations, albeit on nuclear spins. Quantum manipulations on electron states are difficult to observe as they interact with leads. 
(A)
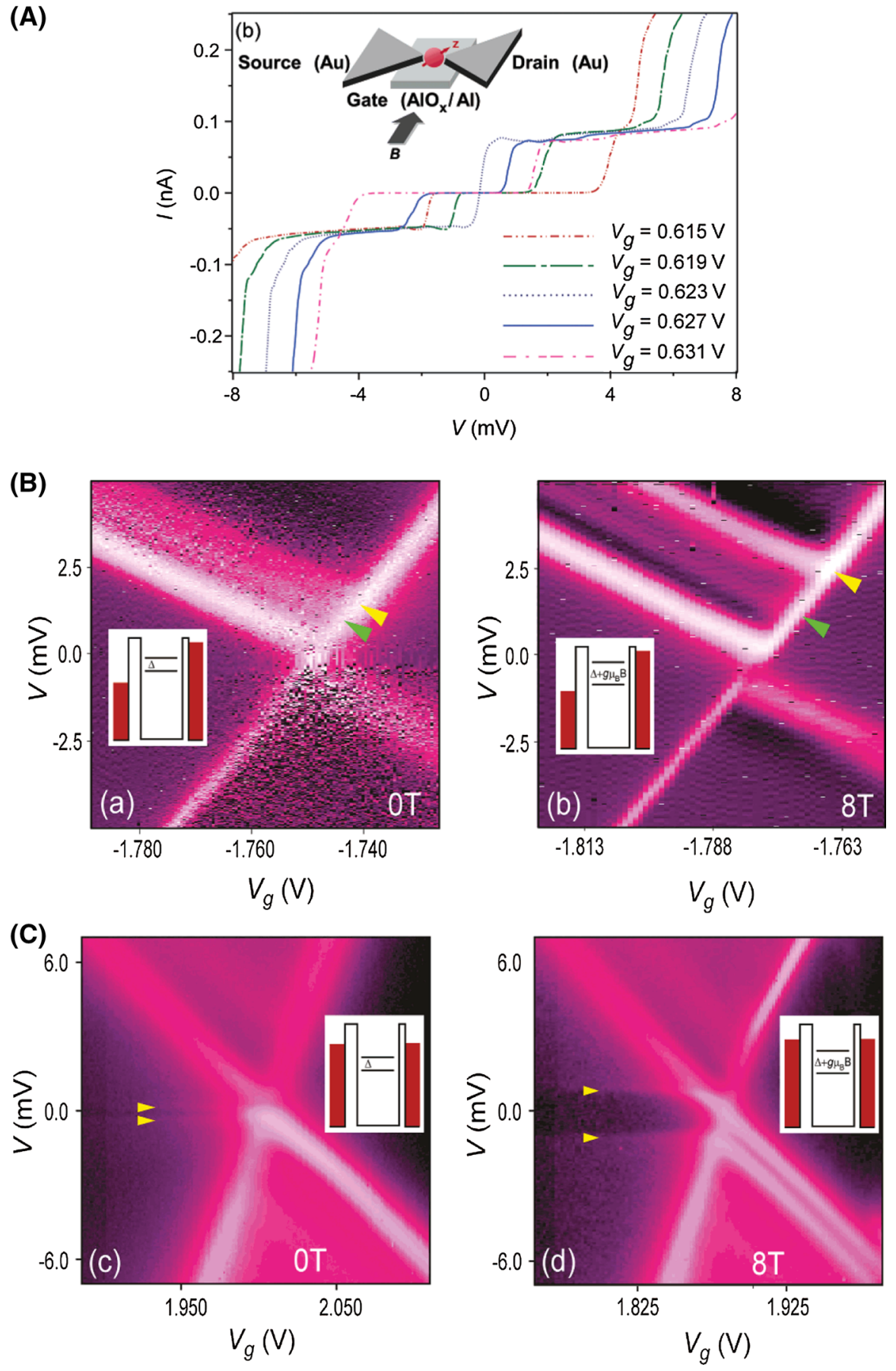

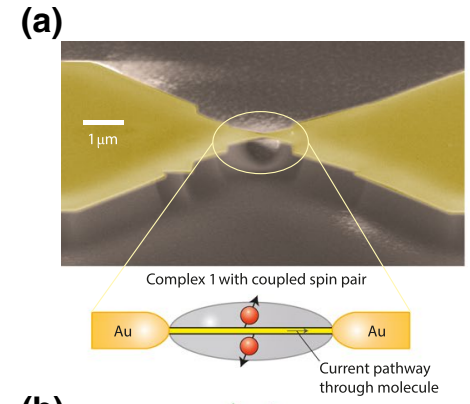

(b)

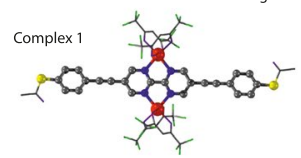

(c)

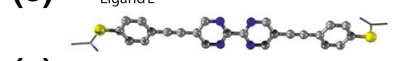

(e)
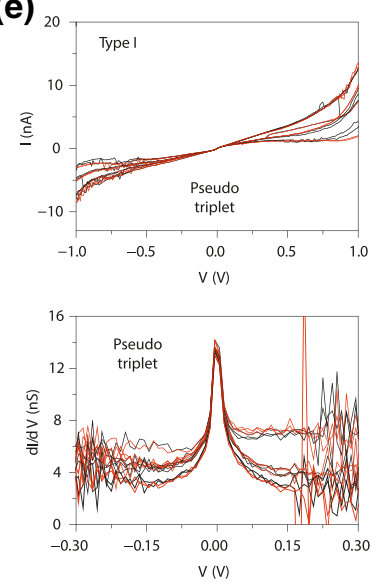
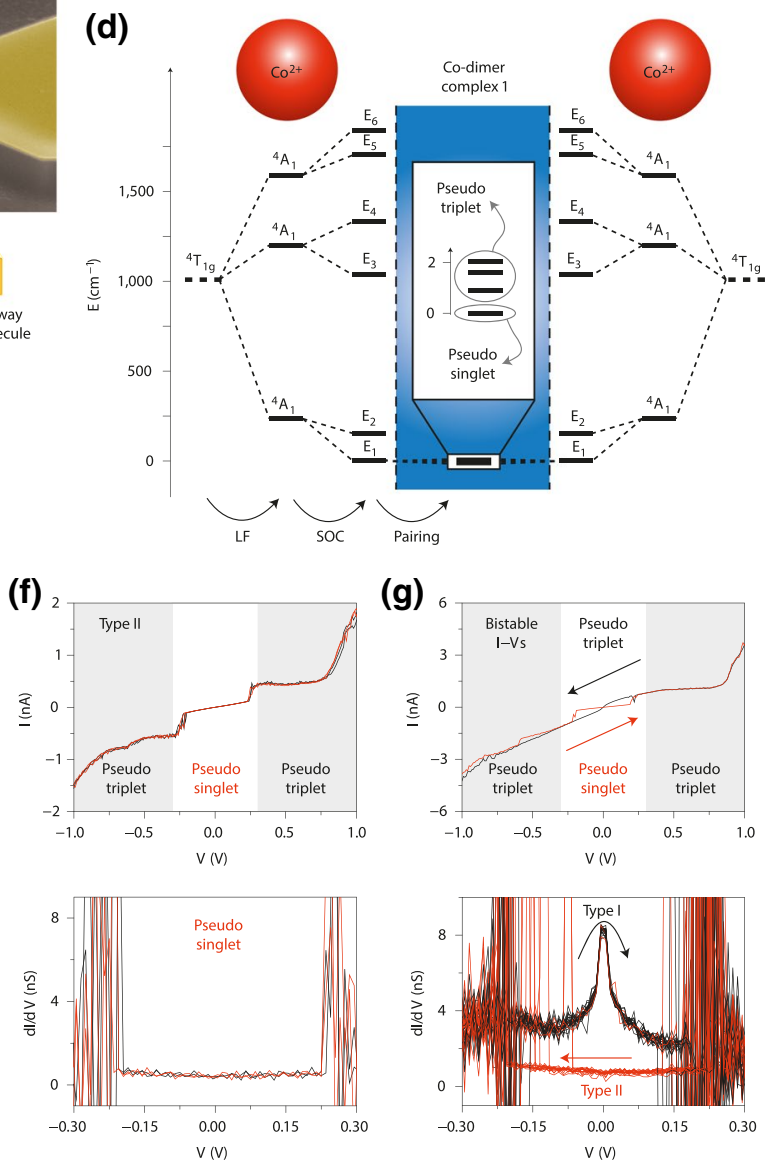

Fig. 13 Dimer coupled to a break junction. a Experimental setup. The co-dimer offers a conduction channel so that electrons do not flow directly through the metallic centers. Therefore, flowing electrons do not affect the oxidation state. b Molecular structure of the dimer. $\mathbf{c}$ Structure of the binding ligand. d Molecular orbital (MO) illustration of the energy levels. The ${ }^{4} T_{1 \mathrm{~g}}$ state is split via ligand-field and spinorbit interactions. Due to pairing, a pseudo-singlet state and a pseudo-triplet state are formed, where the pseudo-singlet state is the ground state. e, f Two $I-V$-characteristics have been repeatedly obtained. Type I (e) shows a zero bias anomaly similar to Kondo behavior (assigned as triplet state), while it does not appear in type II data (f). $\mathbf{g}$ Hysteresis behavior obtained directly after (f). This measurement explains the state assignments in $(\mathbf{e}, \mathbf{f})$. Via distinct voltage sweeping, one can manipulate the coupling between the cobalt ions so that either a triplet or a singlet state is favored. Pictures adapted from Ref. [103]. Reprinted with permission from Springer Nature 

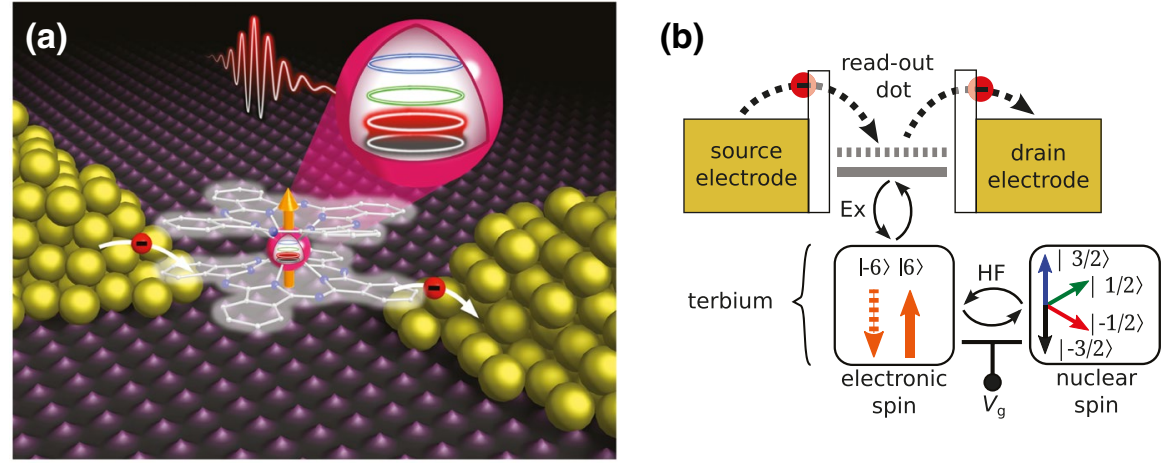

Fig. 14 Setup for pulse experiments on SMMs in break junctions. a Scheme (artist's view) of the sandwiched molecule where the easy-axis (quantization axis) points perpendicular to the plane. $\mathbf{b}$ The flowing electron interacts with the electronic spin of the $\mathrm{TbPc}_{2}$ molecular magnet, which again is coupled to the Tb nuclear spin $(I=3 / 2)$. Picture adapted from Ref. [104]. Reprinted with permission from AAAS
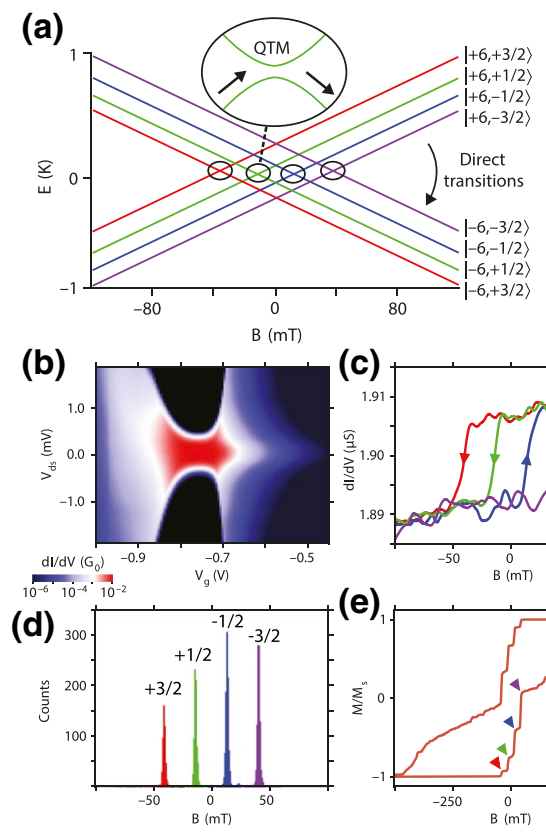

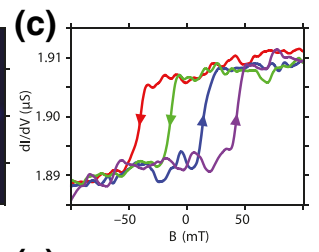

(e)

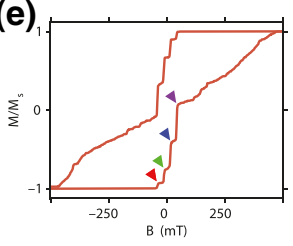

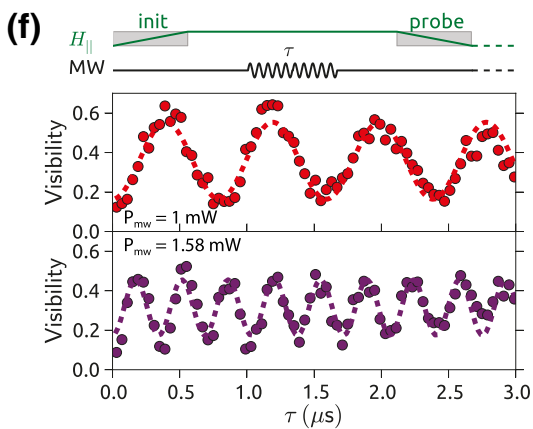

(g)

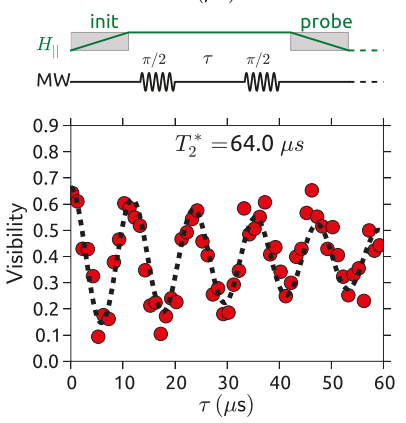

Fig. 15 Spin dynamics of an SMM in a break junction. a Nuclear Zeeman effect of the spin ground state. The avoided crossings indicate quantum tunneling of magnetization (QTM). b Stability diagram of the quantum dot at $0.1 \mathrm{~K}$. For the following measurements, $V_{\mathrm{g}}=0.9 \mathrm{~V}$ and $V=0$ have been chosen. $\mathbf{c}$ Jumps of the conductance when sweeping the field. d Histogram of the conductance jumps for 11,000 field sweeps. The four preferential field values are assigned to QTM. e Normalized hysteresis loops by integrating over 1000 field sweeps showing typical QTM signatures. f Rabi oscillations between $\mid 3 / 2>$ and $\mid 1 / 2>$ states. The microwave pulse creates a coherent manipulation of the two states. $g$ By exciting the system with two $\pi / 2$-pulses that are separated by a time delay $\tau$, the coherence time was determined to $T_{2}^{*}=64 \mu \mathrm{s} . T_{1}$ was determined to $34 \mathrm{~s}$ for the $\mid 3 / 2>$ state, and $17 \mathrm{~s}$ for $\mid 1 / 2>$. Pictures adapted from Refs. $[7,104]$. Reprinted with permission from Springer Nature and AAAS 
(a)<smiles>c1cc2ccc3cccc4ccc(c1)c2c34</smiles>

pyrene

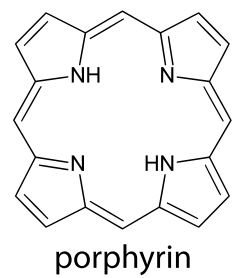

(b)

5)

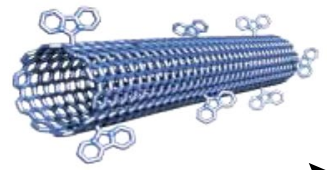

4)

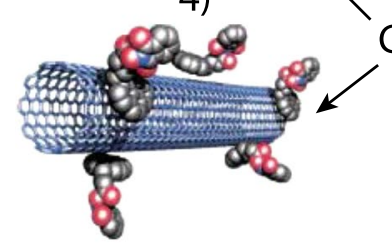

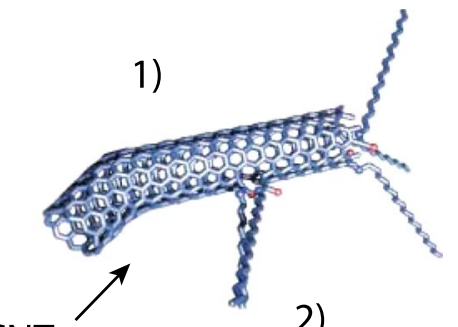

2)

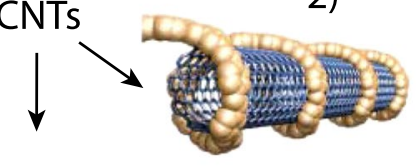

3)

Fig. 16 Covalent and non-covalent binding techniques on CNTs. a Anchoring on CNTs via $\pi-\pi$ stacking of pyrene or porphyrin groups. b (1) Defects within CNTs serve as covalent binding sites. (2) Polymeric groups wrap around CNTs. (3) Fullerenes caught inside the CNT. (4) Stacking of molecules via $\pi-\pi$ or van der Waals interactions. (5) Direct covalent stacking of carbon sites. Picture adapted from Ref. [109]. Reprinted with permission from Wiley-VCH

\subsubsection{Molecular Spintronics on Double-Dot Devices}

The final approach makes use of grafting molecular magnets on low-dimensional substrates such as carbon nanotubes (CNTs) or graphene. The appealing feature of CNTs is definitively its possible integration in FETs where it serves as a conduction channel between the source and drain electrodes. Attaching molecules to the CNT influences the conduction behavior of flowing electrons in different interesting ways.

The anchoring of molecules on CNTs can be achieved either via covalent or non-covalent binding. Covalent binding is enabled via stacking on defects or direct binding on carbon sites (Fig. 16). However, this introduces further scattering centers for electrons and thus degrades the performance of CNT-based devices. In contrast, non-covalent techniques have a way smaller impact on the CNT structure. In many cases, non-covalent stacking techniques imply $\pi-\pi$ interactions. Pyrene or porphyrin materials are well suited to stack molecules on CNTs (see Fig. 16). By attaching pyrene-terminated alkyl chains, $\mathrm{Fe}_{4}$-based SMMs could be successfully grafted on CNTs [106]. Moreover, proteins can be bound on the CNT [107]. Furthermore, van der Waals interactions also proved to be suited, which has been shown for nanoparticles [108]. Encapsulation of $\mathrm{Mn}_{12} \mathrm{Ac}$ SMMs is also reported [12].

The electron flow in CNT-fabricated FETs is manipulated by four main coupling possibilities between the SMM and the CNT, which are illustrated in Fig. 17. The different couplings, however, strongly depend on the type of binding. For 


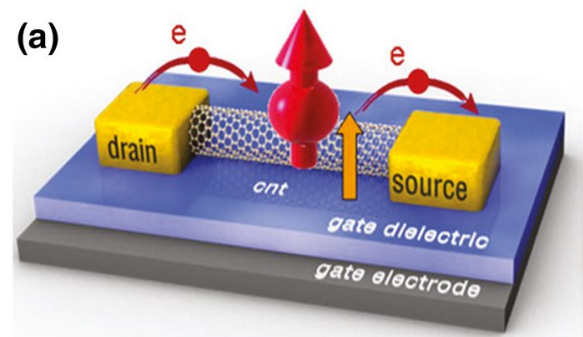

- dipolar coupling

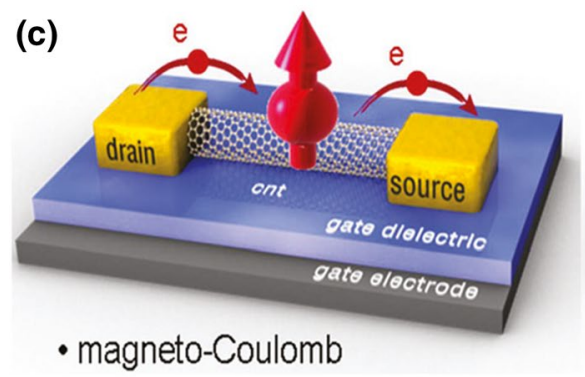

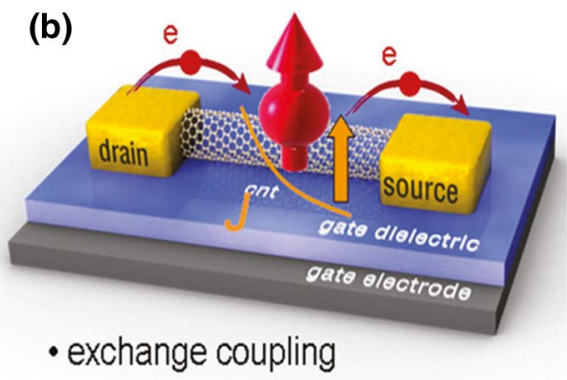

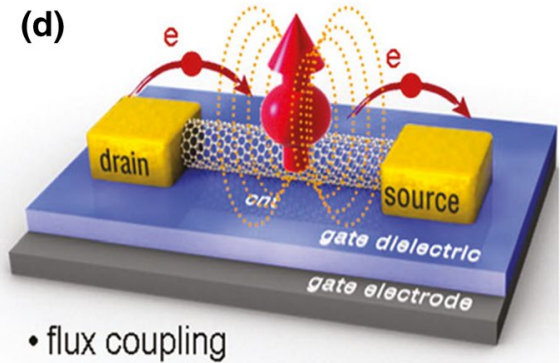

Fig. 17 Different coupling mechanisms between the SMM and the CNT. The QD, which is a CNT in this case, can couple with the SMM via a dipolar interactions, b exchange interactions, c magneto-Coulomb interactions or d via flux interactions. The last case is only observable when the leads are in a superconducting state. Picture adapted from Ref. [29]. Reprinted with permission from the Royal Society of Chemistry

non-covalent binding (so-called weak-coupling regime), dipolar coupling (Fig. 17a) is present. Dipolar coupling leads to a lowering of the conductance since flowing electrons see the SMM as a scattering center [29, 110]. Furthermore, magneto-Coulomb interactions may significantly influence the conductivity. When the spin of the attached molecule is inversed, e.g. by a field sweep, a sudden jump in conductance is observed [111]. This has been attributed to a change of the Zeeman energy which shifts the chemical potential of the CNT. Direct exchange coupling is observed, when two molecules of the same species are attached to the CNT via covalent binding [2]. The covalent functionalization must allow an overlap of the orbital wavefunctions. Finally, the spin center can also interact with flowing electrons via flux coupling. This phenomenon is the one that enables detection with CNT-based superconducting quantum interference devices (CNT-SQUIDs) [112].

Experiments on pyrene-substituted $\mathrm{TbPc}_{2}$-molecules on CNTs reveal a very strong magnetoresistance effect up to $300 \%$ below $1 \mathrm{~K}$ [113]. Therefore, such devices can be used as very efficient spin valves. The measurements are discussed in Fig. 18. Furthermore, nuclear spin-dependent magnetic hysteresis has been observed for similar systems in break junctions [114], which proves the suitability of the double-dot method (see Fig. 19). Ganzhorn et al. investigated the electron-phonon coupling within the nanodevice. Since the SMM is well decoupled from the leads, it can only couple to phonon modes of the CNT. Since phonon signatures appeared as lines 
(a)

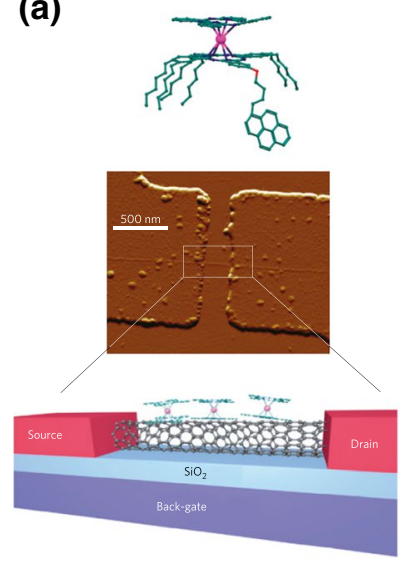

(b)

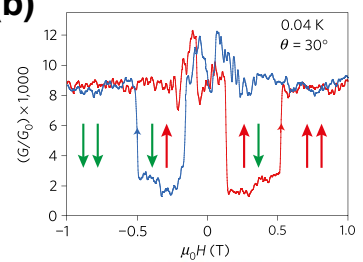

(c)

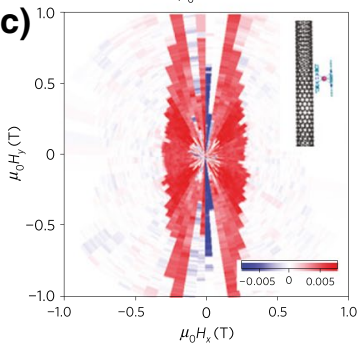

(d)
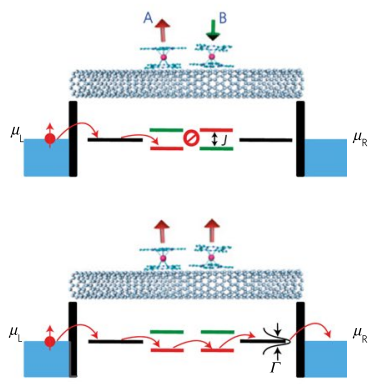

Fig. 18 Giant magnetoresistance in CNTs with pyrene-substituted $\mathrm{TbPc}_{2}-\mathrm{SMMs}_{\text {a }}$ a Experimental setup. The SMM stacks on the CNT via $\pi-\pi$ interactions of the decker ligands. Several molecules are grafted on the CNT surface. b Conductance measurements at $T=40 \mathrm{mK}$ for different field sweeps (red: upsweep, blue: down-sweep). Only in parallel orientation of the molecular spins a high conductance is observed, while a low one appears for the exchange-stabilized antiparallel orientation. c Orientation and magnetic field amplitude dependence of the magnetoresistance. The red region corresponds to bistable regions, while the white one indicates no changes (parallel orientation). The border between the regions marks the critical field. d Illustration of the spin valve phenomenon. Pictures adapted from Ref. [113]. Reprinted with permission from Springer Nature

in the conductance map (Fig. 19d), it was possible to determine the electron-phonon coupling constant, albeit with large error bars. It has to be noted that although the phthalocyanine complexes have proved to be well suited for CNTs, iron- and manganese-based devices are also under investigations $[12,115]$.

Graphene can alternatively serve as a QD device. An FET based on graphene and multiple aggregated $\mathrm{TbPc}_{2}-\mathrm{SMMs}$ have been realized by Candini et al. [117]. They report the possibility of electrically detecting the magnetization state of many SMMs attached to the dot. Furthermore, they were able to determine the magnetic anisotropy just by conductance characteristics. Their results are displayed in Fig. 20.

\subsection{Concluding Remarks}

In the scope of this chapter, we have presented the three prominent ways of integrating molecular magnets into nanoelectronic circuits. The influences of single magnetic molecules on the transport characteristics could be studied and many new coupling phenomena on the quantum level have been observed and are still under discussion. Nevertheless, some points have to be considered when designing spintronic devices.

Surface attachment of SMMs remains an issue. Only a minor part of the rich family of SMMs is suited for one of the three presented contacting methods. The 
(a)

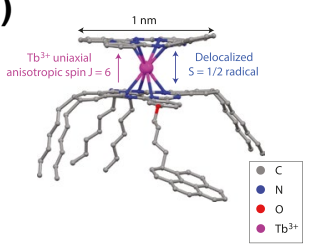

(b)

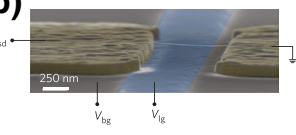

(c)

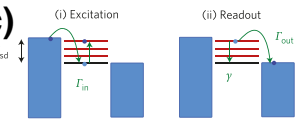

(d)

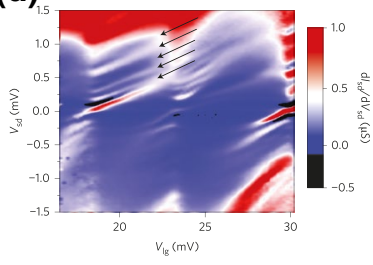

(f)

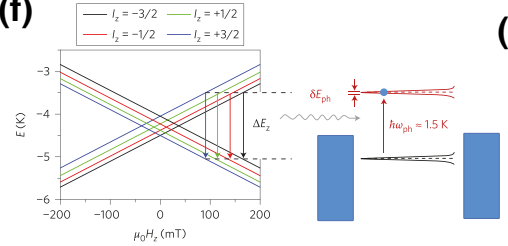

(e)

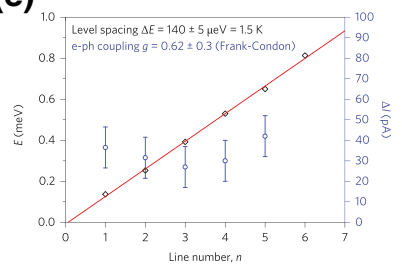

(g)

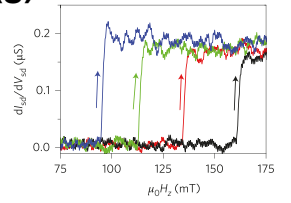

Fig. 19 Electron-phonon interactions in CNTs with pyrene-substituted $\mathrm{TbPc}_{2}-\mathrm{SMMs}_{\mathrm{a}}$ a Chemical structure of the pyrene-substituted $\mathrm{TbPc}_{2}$-SMM. b SEM picture of the CNT-NEM (nanoelectromechanical system) grafted between two leads on a metallic gate (blue) and $\mathrm{Si}++$ backgate (brown). c Single-electron tunneling mechanism (SET). Due to SET, the equilibrium position of the CNT is shifted along its rotational axis [116], which is proportional to the electron-phonon coupling constant. Therefore, the electron is caught in an excited vibrational state (marked red). If the tunneling rate $\Gamma_{\text {out }}$ is larger than the relaxation rate $\gamma$, equidistant signatures parallel to the edges of the Coulomb-diamonds (d), marked with black arrows, are observed. d Stability diagram for different gate $V_{\mathrm{g}}$ and source-drain voltages $V_{\text {sd }}$ obtained at $B=1.4 \mathrm{~T}$ and $T=20 \mathrm{mK}$. e Extracted energies of the vibrational states (black diamonds), fitted with a linear law, at $V_{\mathrm{g}}=18 \mathrm{mV}$. The fit gives a longitudinal stretching mode energy of $\hbar \omega=180 \mu$ eV. From the current intensity, the electron-phonon coupling constant (blue circles) could be determined to $g=0.6 \pm 0.3$, indicating strong coupling. $\mathbf{f}$ Zeeman splitting of the hyperfine-coupled ground state. If the energy difference fits to the phonon energy, the electron relaxes and goes into an excited vibrational state. g Magnetic hysteresis for a magnetic field parallel to the quantization axis of the CNT. The sudden jumps are due to changes of the total spin state. The absolute transition position depends on the populated nuclear spin state. The signature is similar to Fig. 15c. Pictures adapted from Ref. [114]. Reprinted with permission from Springer Nature

magnetic behavior of SMMs is enormously sensitive to their direct surrounding. Electron flow through the spin center may oxidize or reduce the metal ion, which results in totally different magnetic behaviors. Contacting to metal leads may also change the magnetization state, as shown for the archetypal $\mathrm{Mn}_{12} \mathrm{SMM}$. Therefore, a more practical approach is to use less manipulative contacting methods based on $\pi-\pi$-stacking that preserves magnetic properties more likely. Furthermore, current passing close to magnetic molecules rather than directly through the spin center showed to be advantageous, too, which is by the way the opposite of classical spintronics. Therefore, the double-dot approach is the most promising one for the investigation of quantum spintronic effects on the single-molecule level and integration into nanocircuits. Anchoring SMMs in graphene devices could open a new range of possibilities in the near future due to the two-dimensional landscape, with novel architectures and yet unknown interaction phenomena. The interactions with massless relativistic electrons in the Dirac model $[118,119]$ yield an especially fruitful ground for scientists [29].

In general, reproducibility remains a partially unsolved issue. The deposition and grafting processes are not reliable enough in a way that every nanodevice 
(a)

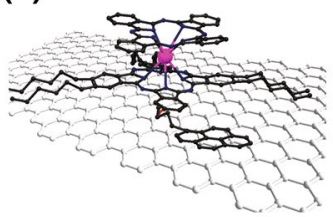

(d)

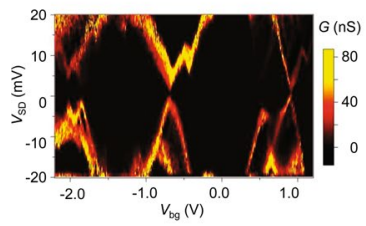

(b)

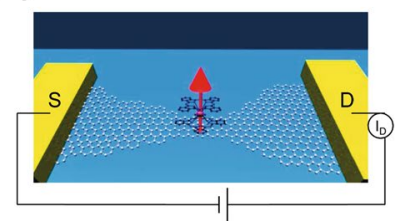

(e)

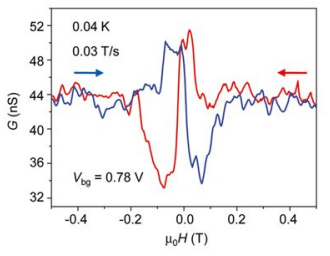

(c)

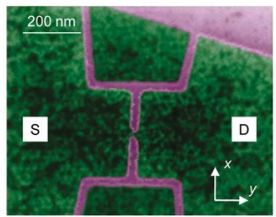

(f)

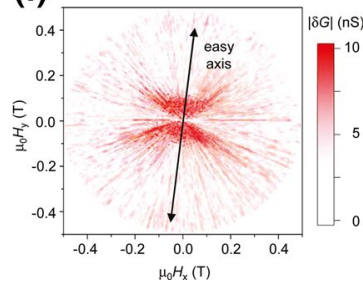

Fig. 20 Graphene FET transistor with a single pyrene-substituted $\mathrm{TbPc}_{2}-\mathrm{SMM}$. a Stacked SMM on a graphene lattice. b Idealized scheme of the FET. c SEM picture of the FET. The purple colour represents the $\mathrm{SiO}_{2}$ substrate and etched graphene. d Conductance mapping as Coulomb-diamonds. The structure indicates that the nanoelectronic device operates in the Coulomb blockade regime. e Conductance hysteresis at a fixed backgate voltage. The hysteresis origin is profoundly discussed in Ref. [117]. The authors ascribe it to magnetization reversions of the SMM at small fields. $\mathbf{f}$ As the hysteresis depends on the easy-axis orientation (as typical for SMMs), a two-dimensional map of the conductance against the field direction reveals the orientation of the molecule, which agrees with crystalline data. Pictures adapted from Ref. [117]. Reprinted with permission from Copyright (2011) by the American Chemical Society

behaves the same. However, by choosing proper ligands, the orientation of molecules can be controlled. Thus, chemical engineering of SMMs is still a very important field for molecular spintronics. Perhaps novel advanced routes in chemistry could solve this problem. Nevertheless, the control over the orientation of the nanoscopic gaps remains challenging.

Although many phenomena have been observed up to now, some of these are not yet fully understood. For instance, it is still unclear why no additional transverse anisotropy terms have been observed in the grafted SMMs, which one would intuitively expect due to the new surrounding [29]. Effects on the manipulated phononic environment have been unclear for a long time, but first promising results have been reported recently [120]. The creation of new theoretical models is thus an important research field.

One goal of molecular spintronics, often mentioned in the literature, is the integration into quantum computational devices, which seems natural as it operates on the atomic level. To perform quantum logic operations, the quantum coherence time, particularly the phase-memory time, must be sufficiently large enough, but is decreased via dipolar and hyperfine interactions rapidly. The path towards quantum coherence measurements of the electron spins have not yet been achieved, but is currently under development. So far, coherence times of nuclear spins have been detected as shown in Sect. 3.2.3. Observed electronic phase-memory times of SMMs usually lie in the (sub-) $\mu$ s-regime (see Sect. 4), which could be even lower when in contact with flowing electrons and would thus 
require demanding readout-techniques. To gain insight the effect of flowing electrons on quantum coherence, new approaches need to be developed.

\section{Pulsed and Transient Electron Paramagnetic Resonance Spectroscopy}

Electron paramagnetic resonance (EPR) has proved to be a great tool to explain the spectroscopic and magnetic behavior of SMMs [8, 11]. In general, EPR covers a wide range of spectroscopic methods, in which the absorption either with or without an external magnetic field is measured in the range of $\approx 1-1000 \mathrm{GHz}$. EPR spectroscopy is a huge research area, and a plethora of techniques have been developed. As we will only discuss a small part here, the interested reader may have a look into Refs. [121-124] for an overview on EPR techniques. This chapter is mainly focussed on pulsed and transient EPR, which give a detailed view on relaxation and spin dynamics of molecules. Combining pulsed EPR techniques with spintronics would yield an unprecedented picture of electron flow effects on magnetization dynamics. Therefore, we review the transient EPR technique, which allows studying perturbations between flowing electrons and spin centers in a well-defined geometry of donor-acceptor molecules.

\subsection{Motivation: Understanding Quantum Coherence in SMMs}

In modern computers, information is stored and processed in forms of two states " 1 " and " 0 ". However, imagining storing information in more than two ways, for example, as any superposition of " 1 "s and "0"s (i.e. $a|1>+b| 0>$ ) as qubits, would greatly enhance computational power, and even overcome current encryption technology with ease $[9,125]$. As theoretically demonstrated by Leuenberger and Loss [10], SMMs are able to perform Grover's quantum search algorithm enabling storage of $2^{2 S+1}$ numbers, thus having some potential applications for quantum computation.

To perform quantum logic operations, the phase relationship must be maintained for a reasonable duration (coherence time $T_{2}$ ) to allow finishing a computation without any errors. Investigation of decoherence mechanisms, such as electron-nuclei or dipole-dipole interactions [126, 127], is crucial for the rational design of SMMs with long coherence times. Pulsed EPR gives direct access to the coherence times, so that decoherence sources can be studied profoundly.

Due to the possibility of controlling and manipulating molecular centers individually, SMMs are interesting objects for integration into quantum computation. The mesoscopic nature together with the large magnetic moment of SMMs enables easier control than atomic objects. Alternative approaches make use of quantum dots [128-130], superconducting qubits [131], entangled states in trapped atomic ions [132] and nitrogen-vacancy centers in diamonds [133, 134], where addressability or the maintenance of quantum behaviors are still important issues [9]. Via pulsed EPR the state of SMMs can be controlled and environmental perturbations investigated. 


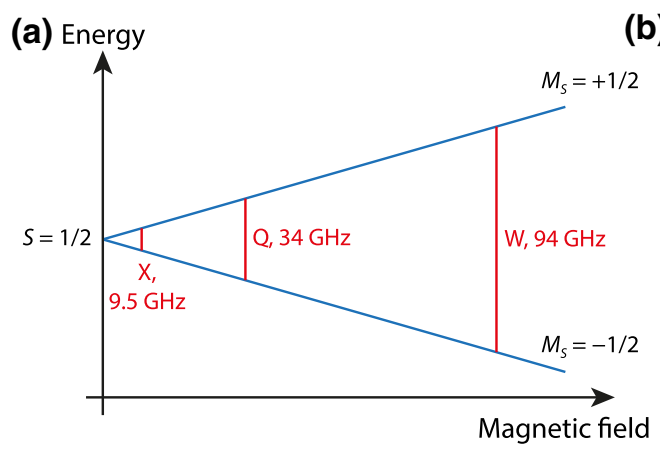

(b) Amplitude

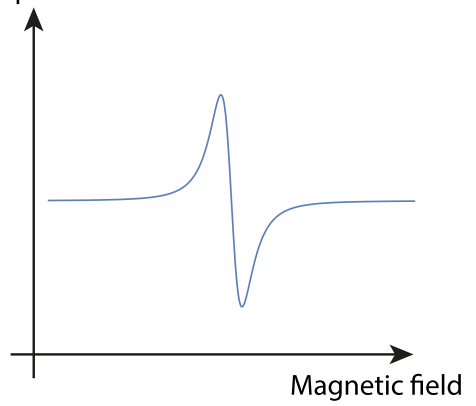

Fig. 21 Energy splitting of a spin-1/2-system in a magnetic field. a The energy splitting scales linearly with the magnetic field in any direction for a spin-1/2-system. However, for $S>1 / 2$ the splitting only scales linearly when the magnetic field is parallel to the quantization axis. The red lines show the transition frequencies. W-band experiments demand approximately ten times stronger magnetic fields compared to X-band experiments, but the stronger splitting increases the resolution by a stronger population difference. b Typical derivative absorption signal of an EPR experiment

The integration of SMM qubits in devices is an important issue for quantum technology. However, control mechanisms are not well developed yet and require innovative approaches. Therefore, integrating pulsed EPR in electronic nanodevices could enable a revolution for quantum computation.

\subsection{Basic Principles of Pulsed EPR}

We consider a spin-1/2-system. The Zeeman effect (Eq. (3)), splits $M_{S}=+1 / 2$ and $M_{S}=-1 / 2$ states as shown in Fig. 21 . Since $M_{S}=+1 / 2$ has a lower energy, it is stronger populated than the excited state following Boltzmann distribution. According to the magnetic dipole selection rules $\Delta S=0$ and $\Delta M_{\mathrm{S}}=0, \pm 1$ [135], the system can be excited by an absorption of a photon. This energy lies typically in the microwave regime.

Frequencies widely used in EPR spectroscopy lie in the X-band (9.5 GHz), Q-band $(35 \mathrm{GHz})$ and $\mathrm{W}$-band $(94 \mathrm{GHz})$ ranges. To fulfill the resonance condition, the field needs to be swept. For a free electron with $g=2.0023$ resonance at those frequencies appears at $0.3390 \mathrm{~T}, 1.213 \mathrm{~T}$ and $3.354 \mathrm{~T}$, respectively. The measured signal typically has a derivative shape due to field-modulation in the measurement process. If the system is continuously irradiated with microwaves at or close to resonance, the population between these two states oscillates. This phenomenon is known as Rabi oscillation [136].

Access to the spin dynamics of magnetic clusters is achieved via time-domain experiments such as pulsed EPR. Furthermore, performing quantum operations is quite similar to pulse EPR techniques, as both work with pulses of variable lengths and phases that match the energy difference between the involved states [11]. In the following we will discuss the phenomena on a qualitative point-of-view. The quantitative analysis of pulsed EPR is carried out extensively in [124] and only final results will be shown here. 


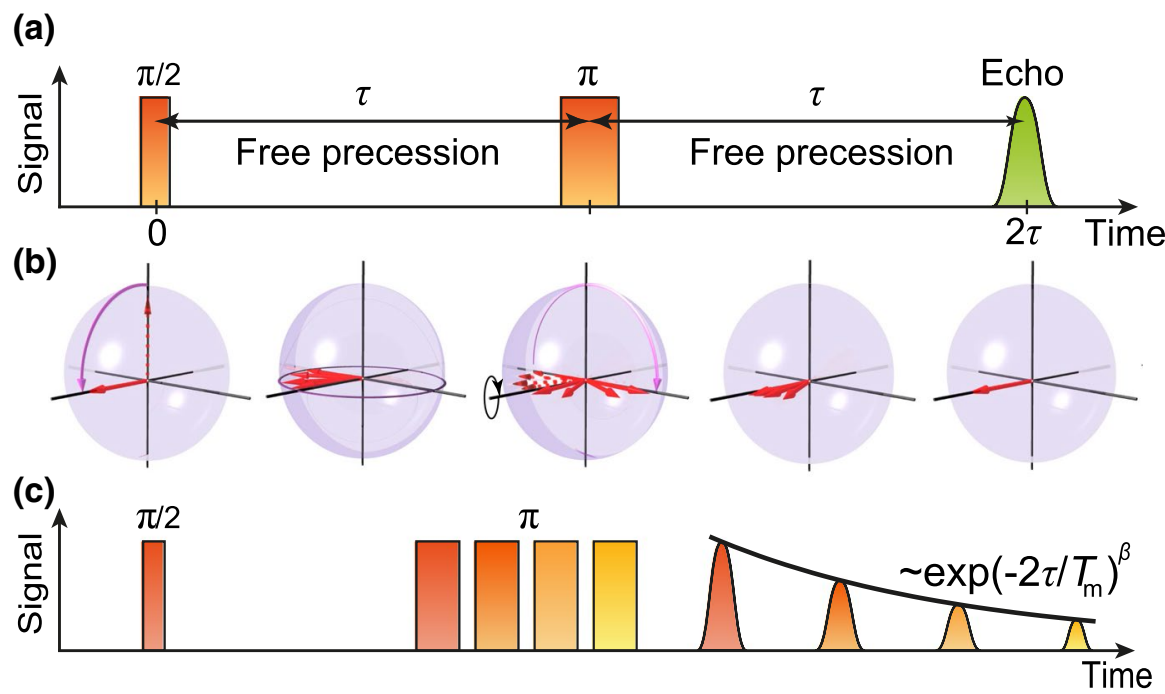

Fig. 22 Hahn-echo experiment in the rotating frame approximation. a The Hahn-echo experiment consists of a $\pi / 2-\tau-\pi-\tau-$ echo pulse scheme. At first, the sample is magnetized in $z$-direction, shown on a Bloch sphere in (b). A $\pi / 2$-pulse polarised in $y$-direction tilts the magnetization into the $x y$-plane, where it evolves freely for a certain time $\tau$. After $\tau$, a $\pi$-pulse adds a phase so that the spins evolve back. When all the single spins align again, which happens after another time delay $\tau$, a signal ("echo") is measured. $\mathbf{c}$ By increasing $\tau$, the echo signal decreases exponentially due to decoherence effects originating from interactions with the environment. The time constant $T_{2}$ denotes the coherence time

One of the simplest pulse schemes in pulsed EPR is the "Hahn-echo" experiment demonstrated in Fig. 22, which is also known as "primary echo" [137]. The following descriptions are discussed in the reference frame of a rotating coordinate system. The Hahn-echo experiment consists of a $\pi / 2-\tau-\pi-\tau$-echo sequence. During the evolution time $\tau$, the magnetization processes in the $x y$-plane and interacts with the environment, which perturbs the spin evolution. After $2 \tau$ the magnetization in $y$-direction follows

$$
M_{y}=M_{y, 0} \cdot \exp \left(-2 \tau / T_{m}\right)
$$

where $T_{m}$ denotes the experimentally accessible phase-memory time, which is a lower limit for the real spin-spin relaxation time $T_{2}$ [11]. Moreover, $T_{2}$ can also be extracted from linewidths of cw-EPR measurements. In contrast, the easiest way to gain the spin-lattice relaxation time $T_{1}$ is via a simple $\pi$-pulse. It can be identified as the exponential time constant in the saturation recovery signal.

\subsubsection{Spin-Lattice Relaxation}

The spin-lattice relaxation time $T_{1}$ originates from interactions of the spin with phonons. In fact, it corresponds to the time to overcome the anisotropy barrier (Fig. 2a), where phonon interactions induce transitions with $\Delta M_{\mathrm{S}}= \pm 1, \pm 2$ [8] thus causing "climbing" of the energy barrier that leads to a nonmagnetic equilibrium. The 
(a) Signal

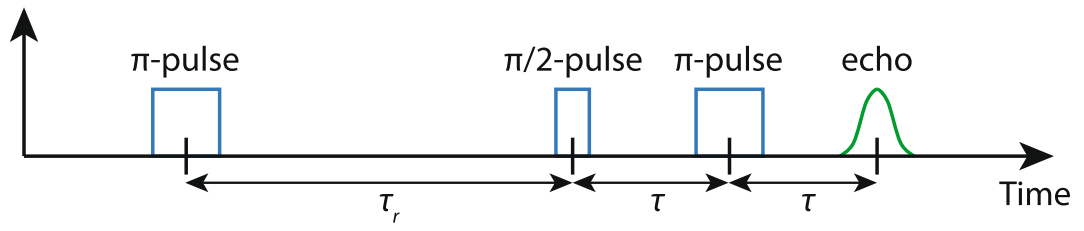

(b) Signal

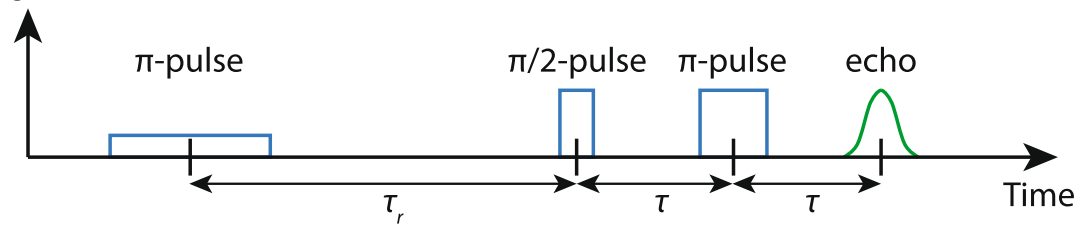

(c) Signal

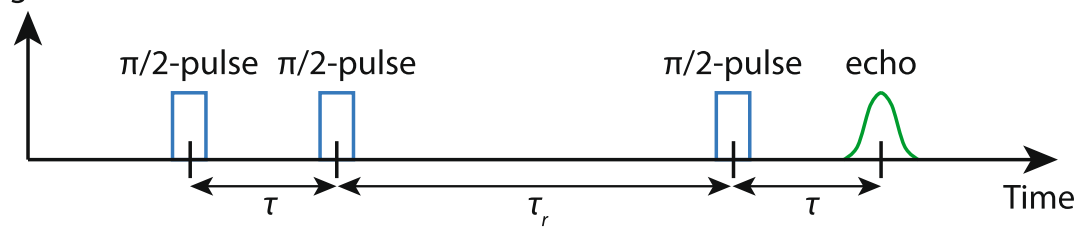

Fig. 23 Pulse sequences for the inversion recovery technique. a Inversion recovery. A $\pi$-pulse initiates the inversion. By varying $\tau_{\mathrm{r}}$ and fitting with Eq. 11 , the spin-lattice relaxation time $T_{1}$ can be extracted. b Echo-detected saturation recovery. c Stimulated-echo decay. Picture adapted after Ref. [138]

relaxation time is temperature-dependent and follows an Arrhenius law (Eq. (1)). The inverse attempt frequency $\tau_{0}$ can be understood as the slowest possible phononinduced spin transition, usually closed to the top of the energy landscape [11].

Access to $T_{1}$ is enabled by performing saturation recovery measurements [124]. In the simplest case, this method uses a single $\pi$-pulse and the signal recovery is measured. More advanced is the inversion recovery technique, which involves a

$\pi-\tau_{r}-\pi / 2-\tau-\pi-\tau-$ echo sequence (Fig. 23a) [141]. The acquired signal then obeys

$$
s\left(\tau_{r}\right)=s_{0}\left[\exp \left(-\tau_{r} / T_{1}\right)-1\right],
$$

where the evolution time $\tau_{r}$ needs to be varied and $\tau$ is fixed, but small. Consequently, $T_{1}$ also limits the repetition rate of the experiment. Furthermore, in the saturation recovery technique effects from spectral diffusion are detected if the inhomogeneous linewidth of the EPR signal is larger than the pulse excitation bandwidth. In this case, deviations from Eq. 11 and a dependence on the excitation pulse duration is observed [138]. Spectral diffusion is caused by molecular tumbling, nuclear spin interaction and cross relaxation [138, 142]. In many cases, spectral diffusion effects can be removed via echo-detected saturation recovery (Fig. 23b), which involves a longer initialization pulse with a lower amplitude [138, 143]. Moreover, via stimulated-echo decay measurements involving a $\pi / 2-\tau-\pi / 2-\tau_{r}-\pi / 2-\tau$-echo 
(a)

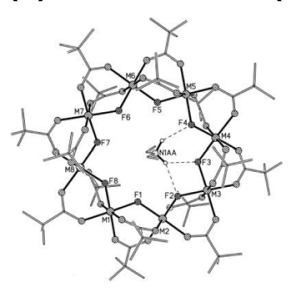

(b)

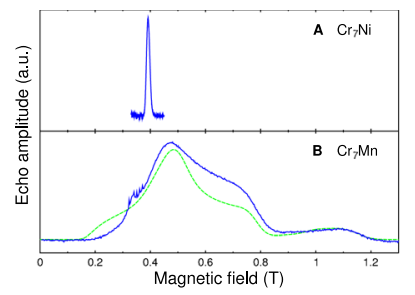

(c)

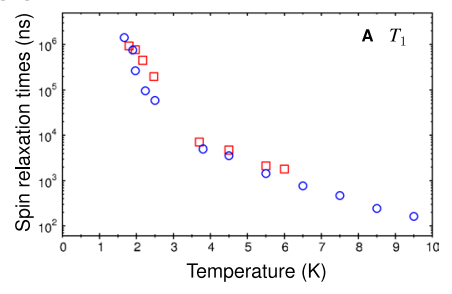

Fig. 24 Structure and $\mathrm{T}_{1}$ of powder samples from the antiferromagnetic rings $\mathrm{Cr}_{7} \mathrm{Ni}$ and $\mathrm{Cr}_{7} \mathrm{Mn}$. a Chemical structure of $\mathrm{Cr}_{7} \mathrm{M}$. b X-band Hahn-echo intensity in dependence of magnetic field measured at $4.5 \mathrm{~K}$. The green curve for (B) corresponds to a simulation with parameters $S=1, g=2, D=21 \mathrm{GHz}$ and $E=1.9 \mathrm{GHz}$. The (B) spectrum is quite broad due to its $S=1$ spin nature and the zero-field splitting that exceeds the microwave frequency. c Temperature dependence of $T_{1}$ for $\mathrm{Cr}_{7} \mathrm{Ni}$ (blue circles) and $\mathrm{Cr}_{7} \mathrm{Mn}$ (red squares). The exponential decay indicates typical phonon-induced relaxation behavior. Remarkably, although the spin is different (1/2 vs. 1), the temperature behavior is almost the same for both compounds. Pictures adapted from Refs. [139, 140]. Reprinted with permission from Wiley-VCH and Copyright (2007) by the American Physical Society

Fig. 25 Inversion recovery measurements on $\mathrm{Cr}_{7} \mathrm{Ni}$ for different concentrations and temperatures. The inversion recovery measurement was performed using a more exotic $\pi-\tau-\frac{2}{3} \pi-\tau_{\text {fix }}-\frac{2}{3} \pi-\tau_{\text {fix }}-$ echo sequence. Picture adapted from Ref. [146]. Reprinted with permission from Copyright (2011) by the American Physical Society

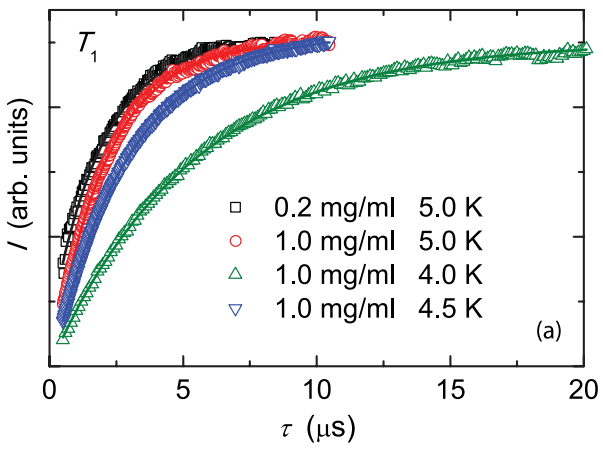

sequence, spectral diffusion effects can even be enhanced [124]. In addition, twophonon-induced relaxation may also play a role. They are classified as Orbach relaxation, where an additional real excited state is involved, or Raman relaxation, where an excited virtual state plays a role [54, 144, 145]. We note that the relaxation kinetics can also be acquired from dynamic magnetometry. By measuring the in-phase and out-of-phase magnetic moment in an alternating magnetic field, the spin-lattice time $T_{1}$ can be extracted [8].

The first molecular magnetic compounds to show quantum coherence via pulsed EPR were the antiferromagnetically coupled rings $\mathrm{Cr}_{7} \mathrm{Ni}$ and $\mathrm{Cr}_{7} \mathrm{Mn}$ with $S=1 / 2$ and $S=1$, respectively [140]. From the results shown in Fig. 24 one can observe the typical Arrhenius-like temperature dependence of $T_{1}$ indicating phonon-induced relaxation. Furthermore, the authors observed a variation of two in $T_{1}$ when choosing different magnetic fields for the measurement, which is due to orientation-selective excitation of parts of the powder. This gives rise to the conclusion that magnetic anisotropy and the related zero-field splitting play an important role in relaxation. Furthermore, a follow-up by Schlegel et al. (see 

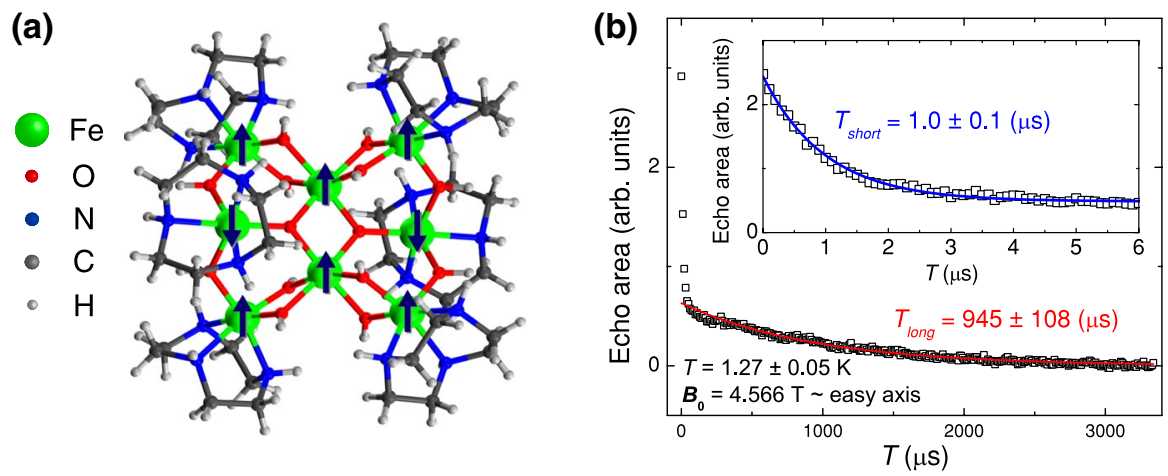

Fig. 26 Determination of spin-lattice relaxation in an $\mathrm{Fe}_{8} \mathrm{SMM}$. a Chemical structure of the $\mathrm{Fe}_{8} \mathrm{SMM}$. b Measured echo intensity from stimulated-echo experiments and fitted relaxation time. Picture adapted from Ref. [150]. Reprinted with permission from Copyright (2009) by the American Physical Society

Fig. 25) at W-band frequency $(94 \mathrm{GHz})$ revealed very similar $T_{1}$ values $(2.9 \mu$ s at W-band (4.5 K) vs. $3.5 \mu$ s at X-band (4.5 K)) [146]. The difference may originate from experimental errors like temperature shifts.

Two-phonon transitions also appear in SMM spectroscopy. The unusual strong temperature dependence of a trinuclear $\mathrm{Fe}_{3} \mathrm{SMM}$ could only be explained by assuming either Orbach or Raman relaxation [147]. Mitrikas et al. concluded that Orbach is more probable, as a Raman fit yields an unusually high fitting exponent.

Furthermore, different relaxation paths also may play a role. Takahashi et al. revealed via stimulated-echo experiments on an $\mathrm{Fe}_{8}$ complex that two relaxation processes with different time-constants $T_{1 \text {,short }}=1 \mu \mathrm{s}$ and $T_{1 \text {,long }}=945 \mu \mathrm{s}$ occur at 1.27 K (see Fig. 26). They attribute the shorter time to spin diffusion and the longer to real spin-lattice relaxation, but they note that it is probably too high compared to the literature values [148, 149].

\subsubsection{Spin-Spin Relaxation and Phase-Memory Times}

The first observations of quantum coherence on SMMs were published around 2000 on $\mathrm{Fe}_{8}$ and $\mathrm{Mn}_{12}$ clusters [151]. However, in this case quantum coherence was not measured via pulsed EPR. Early calculations yielded a lower limit for the coherence time of about $1 \mathrm{~ns}$ in a manganese-based dimer [152]. However, a large step towards possible application of molecular magnetic compounds as quantum logic elements was achieved by Ardavan et al. in 2007. Via pulsed EPR they observed quantum coherence in $\mathrm{Cr}_{7}$ $\mathrm{Ni}$-clusters reaching a phase-memory time of $3 \mu \mathrm{s}$ at low temperatures [140]. This result demonstrates that coherence times can be actually much larger. The basic pulse scheme for determining the phase-memory time is shown and explained in Fig. 22. In subsequent years, many follow-ups both based on both transition metal and lanthanide ions have been reported [146, 147, 150, 153-158], where phase-memory times between hundreds of nanoseconds and 15 microseconds have been observed. 
(a)

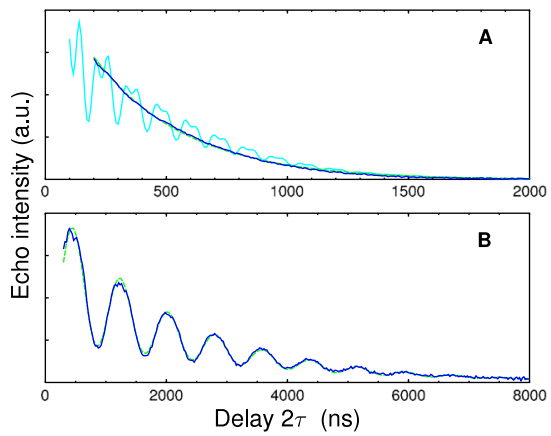

(b)

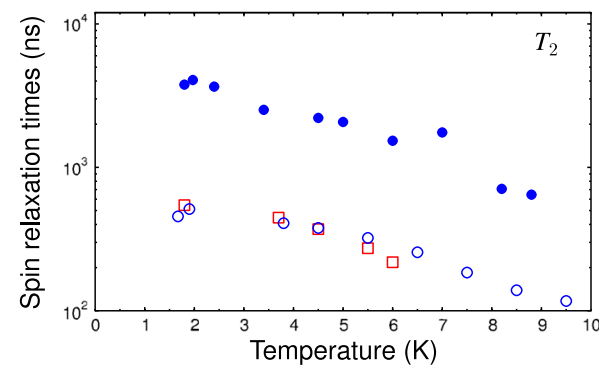

Fig. 27 Hahn-echo sequence on the antiferromagnetic rings $\mathrm{Cr}_{7} \mathrm{Ni}$ and $\mathrm{Cr}_{7} \mathrm{Mn}$. a Decay of the echo intensity upon the time delay for $\mathrm{Cr}_{7} \mathrm{Ni}$. The perdeuterated $\mathrm{Cr}_{7} \mathrm{Mn}$ sample (B) shows a slower decay yielding a longer coherence time. The overlaying oscillations are due to the ESEEM-effect (electron spin echo envelope modulation) $[124,159,160]$. The modulation occurs due to anisotropic hyperfine or quadrupolar interactions with nuclear spins $I>0$, where forbidden transitions with $\Delta m_{I} \neq 0$ are involved. The modulation frequency amounts to $16.6 \pm 0.1 \mathrm{MHz}$. ESEEM can be suppressed using long pulses, as done in (A). The green dashed line represents a fitted curve. b Temperature dependence of $T_{2}$ for protonated $\mathrm{Cr}_{7} \mathrm{Mn}$ (open red squares), protonated $\mathrm{Cr}_{7} \mathrm{Ni}$ (open blue circles) and deuterated $\mathrm{Cr}_{7} \mathrm{Ni}$ (full blue circles). Deuteration enables a much higher coherence time. Figures adapted from Ref. [140]. Reprinted with permission from Copyright (2007) by the American Physical Society

(a)
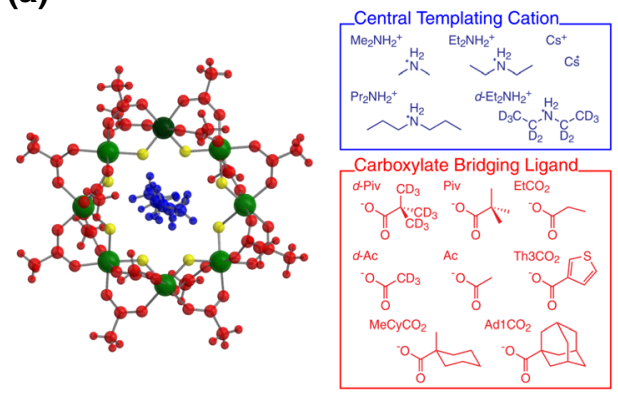

(b)

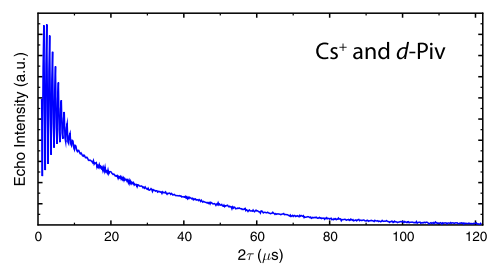

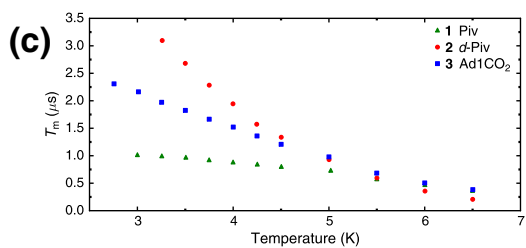

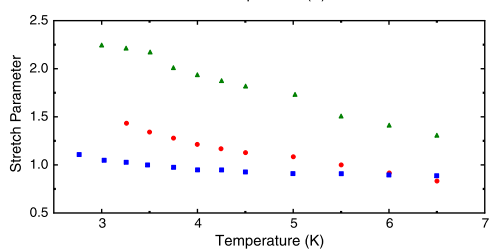

(d)

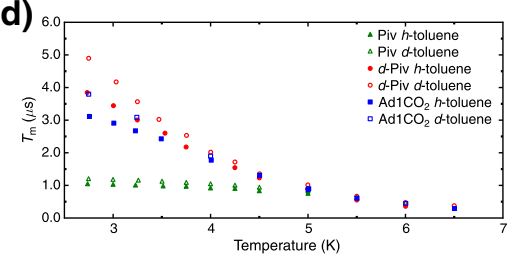

Fig. 28 Enhancing the phase-memory time of $\mathrm{Cr}_{7} \mathrm{Ni}$ by chemical engineering. a Chemical structure of $\mathrm{Cr}_{7} \mathrm{Ni}$ and the different substituents (Cr: green, Ni: dark green, $\mathrm{F}$ : yellow, templating cation: blue, bridging ligands: red). b X-band Hahn-echo decay at $1.5 \mathrm{~K}_{\text {for }} \mathrm{Cs}^{+}$as central templating cation and $d$-Piv as bridging ligand. The extracted phase-memory time amounts to $15 \mu \mathrm{s}$. $\mathrm{c}$ Effects of different bridging ligands on phasememory time $T_{m}$ and stretch factor $x$ in frozen $h$-toluene solution. Deuteration of Piv leads to a huge increase of $T_{m}$ at low temperatures. As cations, $\mathrm{Et}_{2} \mathrm{NH}_{2}^{+}, d-\mathrm{Et}_{2} \mathrm{NH}_{2}^{+}$and $\mathrm{Pr}_{2} \mathrm{NH}_{2}^{+}$, where used for $\mathbf{1}, 2$ and 3, respectively. d Deuteration effects of the solvent on $\mathrm{Cs}^{+}$templated compounds. Picture modified and adapted from Ref. [156]. Reprinted with permission from Copyright (2012) by the American Physical Society 


\subsubsection{Quantum Coherence Mechanisms in $\mathrm{Cr}_{7} \mathrm{M}$}

Ardavan et al. determined a phase-memory times of 379 ns at $4.5 \mathrm{~K}$ for $\mathrm{Cr}_{7} \mathrm{Ni}$ by fitting the $2 \tau$-dependence with an exponential law. The ESEEM modulated signal in Fig. 27 indicates strong interactions between the spin center and the nuclear spin of protons. In fact, the phase-memory time can be increased by deuteration of the sample. Compared to hydrogen, the gyromagnetic ratio of deuterium is six times smaller and therefore the ESEEM frequency is reduced approximately by a factor of six, giving $2.556 \pm 0.005 \mathrm{MHz}$ from the fit. At the same time, the phase-memory time is enhanced sixfold giving $2210 \pm 20 \mathrm{~ns}$. Therefore, main decoherence source are protons in this case.

Further investigations showed that the phase-memory time of $\mathrm{Cr}_{7}$ can be increased to $15 \mu \mathrm{s}$ by chemical engineering [156] (see Fig. 28b), including variations of the central templating ligand and carboxylate bridging ligands. To account for a distribution of relaxation dynamics, the fitting function has been modified to

$$
s(2 \tau)=s_{0} \exp \left(-\left(2 \tau / T_{m}\right)^{x}\right),
$$

where $x$ denotes the stretching factor. A stretching value close to 1 indicates spin diffusion, while $x>1.5$ is a hint for nuclear spin diffusion, where protons are susceptible to positional disorder [156]. Spectral diffusion due to rotation and libration of methyl groups in molecule 1 lowers $T_{m}$ significantly (Fig. 28c). Bond vibrations do not play a role at the investigated low temperatures. Deuteration leads to the same result as discussed before [140]: the weaker dipolar coupling of D leads to less spin-flips of electrons thus increasing $T_{m}$. Compound $\mathbf{3}$ has an increased rigidity giving rise to an increase of $T_{m}$ compared to compound $\mathbf{1}$. Although the nuclear spin-flop rate is small $(\approx 10 \mathrm{kHz})$, the coupling to a large nuclear spin bath may increase decoherence [157]. Deuteration of the solvent has marginal effects on the alkyl-ammonium complexes, but considerable ones on $\mathrm{Cs}^{+}$-templated compounds (see Fig. 28d).

(a)

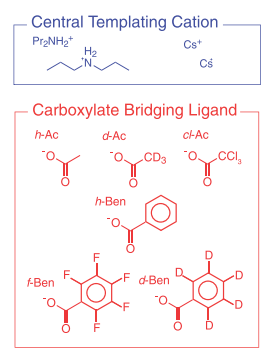

(b)

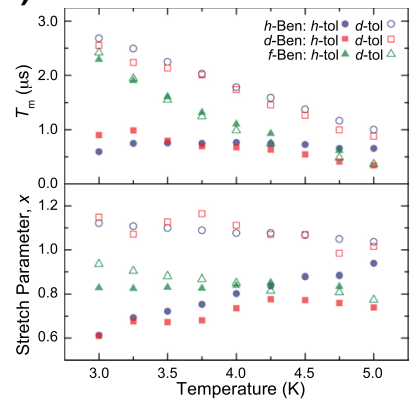

(c)

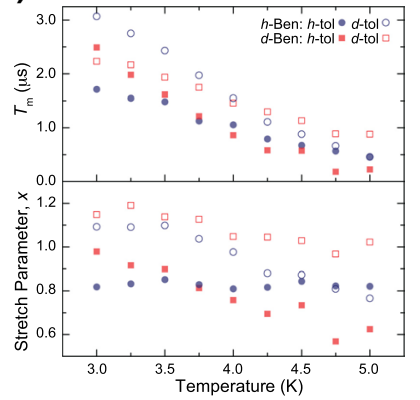

Fig. 29 Halogen-modified $\mathrm{Cr}_{7} \mathrm{Ni}$. a Structure of investigated ligands. b Deuteration of the solvent does not show relevant effects on $T_{m}$ for the fluorine bridging ligands (alkyl-ammonium-templated compound $\mathrm{Pr}_{2} \mathrm{NH}_{2}^{+}$). c Effect of deuteration on the $\mathrm{Cs}^{+}$-templated compound. Picture modified and adapted from Ref. [157]. Reprinted with permission from Copyright (2014) by the American Physical Society 
A more recent study investigates the effect of halogen-modification on $\mathrm{Cr}_{7} \mathrm{Ni}$ (Fig. 29) [157]. ESEEM was observed for deuterated toluene at the characteristic deuterium Larmor frequency, due to the small magnetic moment of D compared to ${ }^{1} \mathrm{H}$ and ${ }^{19} \mathrm{~F}$. Due to the heavier ${ }^{19} \mathrm{~F}$ atoms, spectral diffusion via rotation and libration of the methyl groups of the ammonium cation is less probable. $T_{\mathrm{m}}$ therefore increases with decreasing temperature continuously reaching similar values as for $h$-ben and $d$-ben in $d$-toluene. The hypothesis is supported by comparing the results with the $\mathrm{Cs}^{+}$-templated compound, which seems to not affect coherence (see Fig. 29c).

Measurements at $W$-band frequencies reveal that the interactions between the electron and the proton spins are dipolar. The coupling strength is around $2 \mathrm{MHz}$ [146]. Thus, the coupling to protons represents the largest decoherence path in $\mathrm{Cr}_{7} \mathrm{Ni}$ rings.

More interesting for applications are measurements in the solid phase, but the interactions with neighboring molecules may efficiently decrease $T_{m}$. Therefore, a $\mathrm{Cr}_{7} \mathrm{Zn}$-ring compound with $S=3 / 2$ was played as dopant into a diamagnetic $\mathrm{Ga}_{7} \mathrm{Zn}$ host [161]. Depending on the probed transitions selected by different magnetic fields, phase-memory times of $870 \mathrm{~ns}, 660 \mathrm{~ns}$ and $580 \mathrm{~ns}$ were observed at $2.1 \mathrm{~K}$. Embedding molecular magnets in diamagnetic matrices is thus a promising way to find new possible molecular that directly show quantum coherence in the solid state, albeit the total spin density is reduced.

\subsubsection{Further Examples}

Ideal investigation conditions for the $\mathrm{W}$-band regime is given by an $\mathrm{Fe}_{4} \mathrm{SMM}$ [146], where the zero-field splitting between the lowest $\left(M_{\mathrm{S}}=5\right)$ and the first excited state $\left(M_{\mathrm{S}}=4\right)$ amounts to $92.4 \mathrm{GHz}$, which is close to the central W-band frequency of $94.3 \mathrm{GHz}$. The phase-memory time could therefore be measured without an external field, while measurements with field showed ESEEM modulation of the signal. In this case, coupling to protons of the molecular magnet does not lead significantly to decoherence, but the influence of the solvent is stronger. Exchanging the solvent by $\mathrm{CS}_{2}$, which has almost no nuclear spin, increased the phase-memory time by a factor of two.

In another example of a $V_{15}$-based SMM, local hyperfine coupling to vanadium with $I=7 / 2$ drives the main decoherence path. More interestingly, only the excited state with $S=3 / 2$ shows quantum coherence, while the ground state with $S=1 / 2$ does not $[155,162]$. Furthermore, quantum coherence studies on molecular V(IV)-based complexes $(S=1 / 2)$, comprising a carbon-sulfur scaffold, have attracted great interest. First results showed coherence times in the submicrosecond regime at $10 \mathrm{~K}$, when dissolved in the, almost, nuclear-spin free solvent $\mathrm{CS}_{2}$, which forms a glassy matrix [163]. Measurements on different nuclear spin-free V(IV)-based complexes, dissolved in the polar non-glassy solvent $\mathrm{SO}_{2}$, showed a similar range [164]. Via chemical methods, it was possible to investigate the distance dependence of decoherence caused by nuclear spin-bearing propyl groups. Interestingly, it was found that upon reducing the distance from 6.6 to $4 \AA$ coherence times increased strongly [165]. This indicates that nuclear 
spin-bearing nuclei close to the V(IV) metal center do not contribute to decoherence. Furthermore, charge densities of these complexes seem to play a role in decoherence [166].

It is envisaged to reach large coherence times not only at cryogenic, but also at room temperature. Here, coherence times are limited by spin-lattice relaxation. Therefore, it is also important to investigate the effects of vibronic modes and spin-phonon interactions on decoherence, which act over a broad temperature range. In vanadyl complexes, it was found that magnetic fields can have minor effects on $T_{1}$ times, so that higher frequencies, which require larger fields, can be used in such potential molecular qubits [167]. This is, however, not always the case [168]. Moreover, vanadyl complexes show a strong spin-phonon bottleneck effect, where $T_{1}$ times vary over orders of magnitudes depending on the crystallite size [169]. In a comparable studies with a Tris-chelated vanadium complex, vanadyl complexes outshine them. One reason is the larger phononic excitation energy of the $\mathrm{V}-\mathrm{O}$ bond compared to an $\mathrm{V}-\mathrm{S}$ bond. The exact mechanisms are still under discussion and thoroughly analysed in Ref. [170].

Eventually, it is the goal to reduce the efficiency of spin-phonon interactions. A combination of experimental and theoretical design [170, 171], and knowledge

(a) Spin series (S)

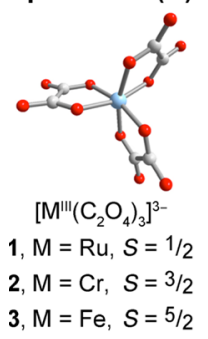

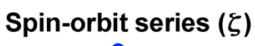

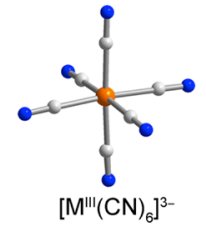

4, $\mathrm{M}=\mathrm{Fe}, \zeta=464 \mathrm{~cm}^{-1}$

5, $M=R u, \zeta=880 \mathrm{~cm}^{-1}$

6, $M=$ Os, $\xi=3100 \mathrm{~cm}^{-1}$ (b)

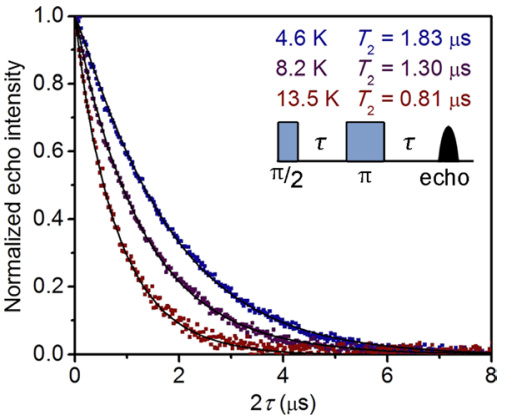

(c)

\begin{tabular}{|c|c|c|c|}
\hline & 1 & 2 & 3 \\
\hline$S$ & $1 / 2$ & $3 / 2$ & $5 / 2$ \\
\hline$H_{\mathrm{dc}}(\mathrm{Oe})^{b}$ & 2812 & 2130 & 3501 \\
\hline$T_{2}$ at $5 \mathrm{~K}$ & $3.44(1)$ & $2.79(3)$ & $1.83(1)$ \\
\hline$T_{2}$ at $14 \mathrm{~K}$ & $2.01(1)$ & $1.86(3)$ & $0.81(1)$ \\
\hline \multirow[t]{2}{*}{$T_{2}$ at $22 \mathrm{~K}$} & $0.41(2)$ & $1.27(4)$ & $0.45(5)$ \\
\hline & 4 & 5 & 6 \\
\hline$S$ & $1 / 2$ & $1 / 2$ & $1 / 2$ \\
\hline$\zeta\left(\mathrm{cm}^{-1}\right)^{c}$ & 464 & 880 & 3100 \\
\hline$H_{\mathrm{dc}}(\mathrm{Oe})^{b}$ & 3364 & 3394 & 3865 \\
\hline$T_{2}$ at $5 \mathrm{~K}$ & $2.38(6)$ & $2.55(4)$ & $4.12(6)$ \\
\hline$T_{2}$ at $13 \mathrm{~K}$ & $0.55(8)$ & $1.25(5)$ & $3.17(4)$ \\
\hline$T_{2}$ at $22 \mathrm{~K}$ & $0.60(9)$ & $1.29(10)$ & $1.04(4)$ \\
\hline
\end{tabular}

Fig. 30 Influence of spin and spin-orbit coupling on decoherence. a Investigated compounds varying spin $S$ and spin-orbit interaction $\xi$, respectively. b Echo intensity decay for compound $\mathbf{3}$ in water/glycerol at different temperatures. c Fitting results from echo intensity decays. Pictures and table adapted from Ref. [175]. Reprinted with permission from Copyright (2014) by the American Chemical Society 
on chemical building blocks [172], has the potential to create potent molecular qubits that can be used for spintronic applications [173, 174].

\subsubsection{Influence of Spin-Orbit-Coupling and Spin State on Phase-Memory Time}

In the previous section, we mainly discussed the influence of nuclear spin bath of the surrounding. Generally, both the spin state itself and spin-orbit coupling are crucial points for the design of possible molecular magnetic materials that show quantum coherence. Graham et al. performed investigations on $T_{2}$ for two compound series, where either the spin state or the spin-orbit interaction at fixed spin is varied (see Fig. 30) [175]. The general trend for the spin series shows a decrease in $T_{2}$ for increasing spin. The authors note that this is due to increased intermolecular dipole interactions and stronger coupling to the nuclear spin bath. However, the maximum $T_{2}$ at $22 \mathrm{~K}$ shows compound 2 , which we attribute to the $90 \%$ abundance of ${ }^{50} \mathrm{Cr}$, ${ }^{52} \mathrm{Cr}$ and ${ }^{54} \mathrm{Cr}$ with no nuclear spin thus almost cutting off one decoherence path.

The spin-orbit series measurements reveal an increase of $T_{2}$ with increasing spin-orbit interaction, which is counterintuitive since spin-orbit coupling mediates spin-phonon relaxation and would therefore introduce another decoherence pathway [175]. The authors concluded that spin-orbit interaction is minorly involved in decoherence, and interactions with the nuclear spin bath are thus the driving force for decoherence. They supported their conclusion with respect to $T_{2}$ of compound $\mathbf{1}$, which shows the largest $T_{1}$ in the spin series despite having the largest spin-orbit coupling. Perhaps spin-orbit coupling is even responsible for their observations, but the concrete coupling mechanism is not yet well understood.

In the investigations shown, we have seen many different interactions that lead to decoherence. However, it is not possible to generate a recipe for maximizing

(a)

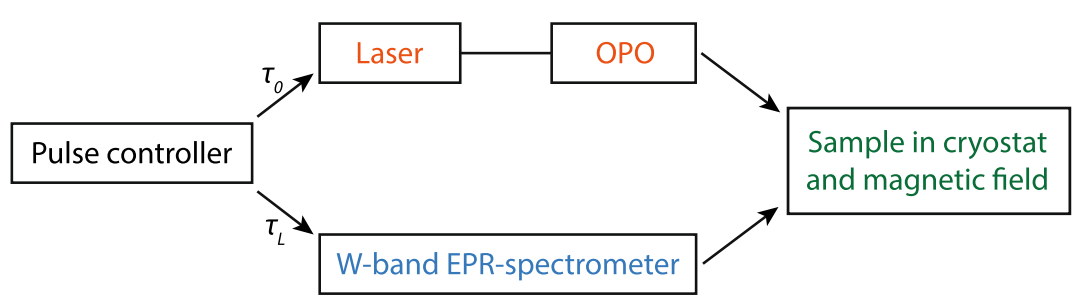

(b) Signal

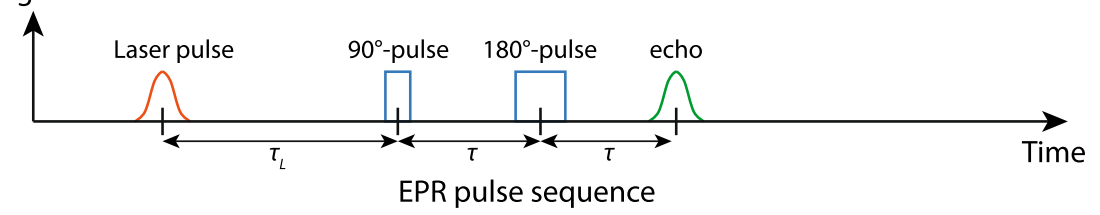

Fig. 31 Time-resolved transient EPR scheme. a A pulse controller triggers both the pulsed laser and the W-band spectrometer. It provides the possibility to set arbitrary delays between the pulses. Alternatively, the trigger of the laser can be used to trigger the spectrometer. The OPO (optical parametric oscillator) enables tuning of the wavelength. The sample is placed in a cryostat in an external magnetic field. b Hahn-echo sequence after initialization with a laser pulse. Scheme adapted after Ref. [179-181] 
coherence. We have seen that decoherence mechanisms playing an important role in one compound may be negligible for another and vice versa ( $\mathrm{see}_{\mathrm{Cr}} \mathrm{Ni}$ and $\mathrm{Fe}_{4}$ investigations). Every case has to be treated differently. Nevertheless, the publications showed how the single influences can be analytically determined and controlled by chemical engineering. However, the effect of flowing electrons on phase-memory times has not yet been studied. An approach towards electron flow interactions can be transient EPR, which is a powerful tool for studying biological samples and solar cells.

\subsection{Transient EPR (TrEPR)}

Transient, which is the opposite of persistent, generally denotes processes that involve non-steady state and produce a decaying signal. The term "transient EPR" denotes EPR experiments, where the interesting properties are induced with light [176-178]. The typical transient EPR scheme is depicted in Fig. 31. Due to its novelty for molecular magnetism, we will first have a look on the possibilities TrEPR offers in other disciplines and draw perspectives for molecular spintronics with SMMs. Here, donor-acceptor dyads and triads, where in the latter a molecule is sandwiched between the donor and the acceptor, play key roles.

\subsubsection{TrEPR in Solar Cell Research}

TrEPR can be used to determine the charge separation dynamics within organic solar cells. Here, the laser pulse first generates a Frenckel exciton and the exact pathways of charge separation can be followed using extensive TrEPR studies. A deep investigation on Si- and C-PCPDTBT:PCBM organic solar cells has been performed by Kraffert et al. [182]. By characterizing both the single precursors and their blends

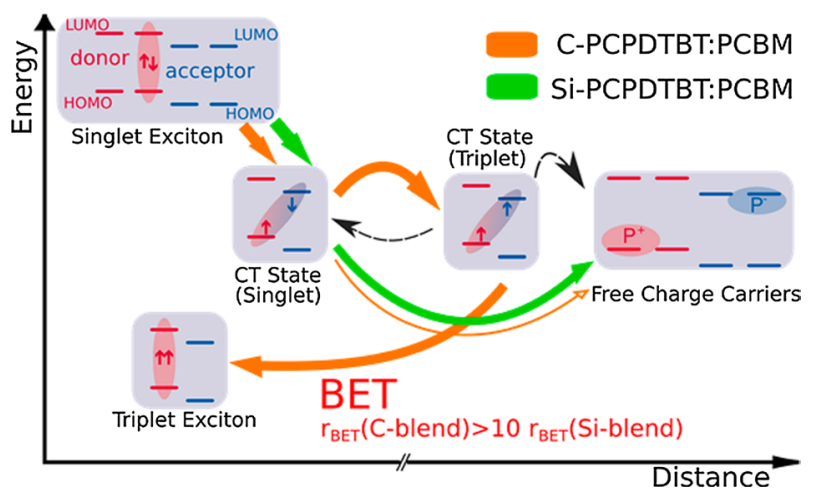

Fig. 32 Charge dissociation in PCPDTBT:PCBM organic solar cells. The photo-excited exciton is efficiently separated in the Si-blends, but much less in the C-blends. In fact, C-blends tend to create triplet exciton states instead of free charge carriers. Therefore, such C-blends are less interesting for application in solar cells. Picture adapted from Ref. [182]. Reprinted with permission from Copyright (2014) by the American Chemical Society 
(A)
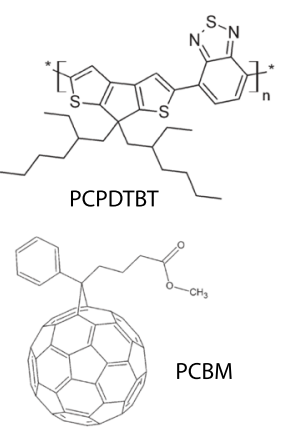

(F)
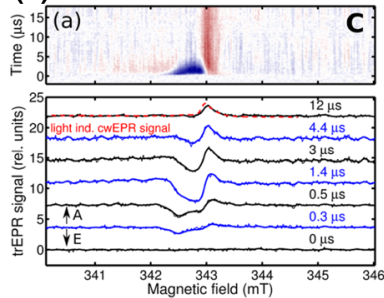

(G)
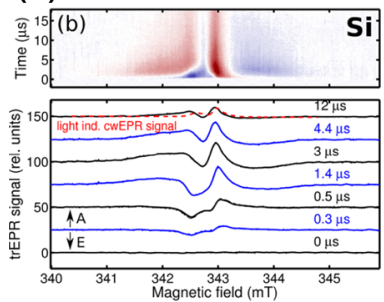
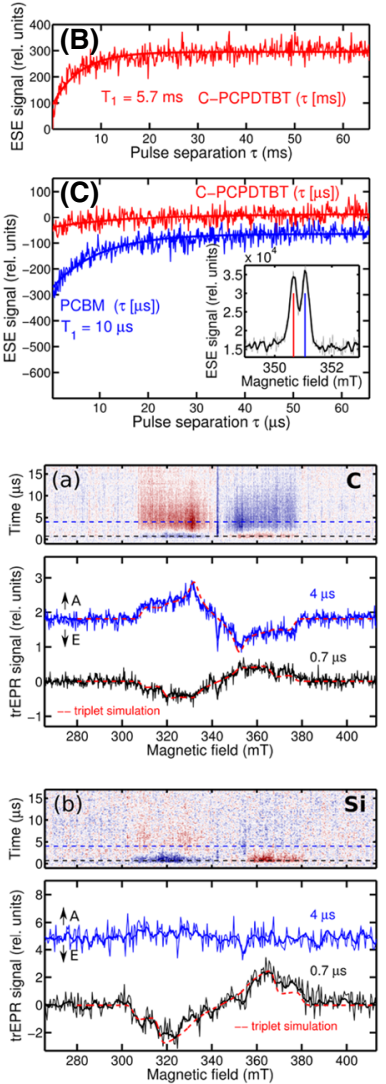
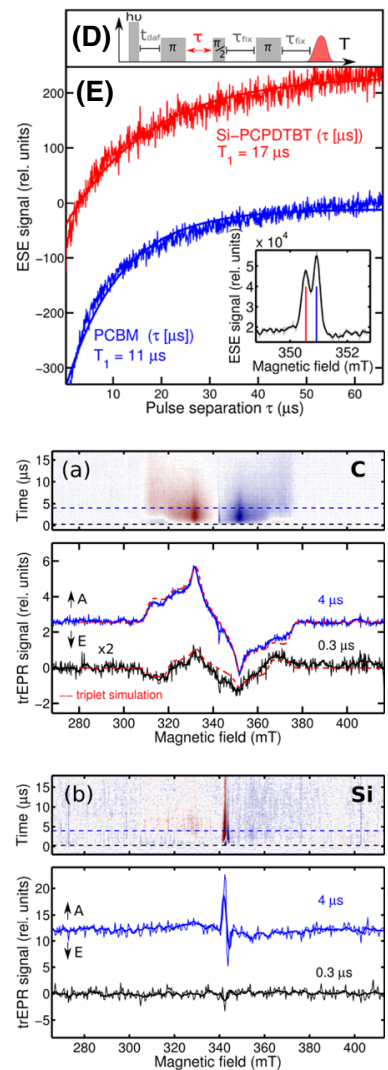

Fig. 33 TrEPR on organic solar cells. a Molecular structure of PCPDTBT (donor), blended with PCBM (acceptor). b-e Inversion recovery measurements reveal a two orders of magnitude larger $T_{1}$ for C-PCPDTBT than for Si-PCPDTBT. Here, $T_{1}$ represents the spin-lattice relaxation involving light-induced polarons. The authors attribute the much higher relaxation times to trapped polarons at defect sites of the C-blend. f, $\mathbf{g}$ CW-EPR after laser excitation (E: emissive signal, A: absorptive signal). The left figures show the evolution of the narrow-bandwidth X-band signal, indicating charge transfer (CT) process induced by light. The CT comes along with generation of spin-correlated polarons, giving rise to an emissive signal for small time delays. For longer delays, the spectrum is purely absorptive. The centered graphs measured on pristine PCPDTBT (C and Si) show typical 70-mT-wide triplet signatures (dotted lines $=$ simulations). The graphs on the right show the signal for thin films of PCPDTBT:PCBM blends. For Si-PCPDTBT:PCBM, the triplet signal is minimal due to efficient exciton dissociation. Pictures adapted from Refs. [182, 197]. Reprinted with permission from Wiley-VCH and Copyright (2014) by the American Chemical Society

for different magnetic field and time ranges (see Fig. 33), the authors were able to follow the dissociation route of a photo-excited exciton. The Si-blends tend to form free charge carriers, while in the C-blends electron back transfers (BET) dominate [183-185], leading to in a low net efficiency as shown in Fig. 32.

In this study, the possibilities offered by TrEPR are fully shown. The TrEPR spectra display a significant difference between intersystem-crossing (ISC) generated triplet states and BET triplets due to different populations of the subspecies [182, 186, 
187]. The BET process follows a signature shown in the right graph in Fig. 33f for $0.3 \mu$ s delay, where either $\left|T_{0}\right\rangle$ or $\left|T_{+}\right\rangle$and $\left|T_{-}\right\rangle$are predominantly occupied, while ISC-generated triplet population depends on the zero-field splitting. With the help of the Easyspin toolbox for Matlab [188] the authors were able to simulate the time evolution of the triplet signal.

For further characterization of solar cells, new methods such as electrically detected magnetic resonance (EDMR) are under development [189]. EDMR is an ideal technique for any conductive material as magnetic resonance is detected by conductivity changes [190]. The most important benefit is its high sensitivity with samples below 100 spins having been tested [191-193]. The interested reader can find an overview on the different EDMR techniques in Refs. [194, 195]. First results on transient EDMR showed good agreement with TrEPR experiments [196]. We foresee that this technique could be a promising tool to observe charge dynamics in molecular spintronic elements with photoexcitable or photoswitchable molecular components.

\subsubsection{TrEPR in Biochemistry}

The interest in understanding the microdynamics of photosynthesis, which is one of the most important processes in nature for our life, motivated the development of the first TrEPR setups [176, 177]. A protein of central importance is the photosystem II complex, in which water-splitting takes place [198, 199]. TrEPR helped to identify in which chlorophyll the photogenerated triplet state is located and finally gave rise to its relative orientation by analysis of the anisotropy tensor [200]. Furthermore, various studies enabled distance measurements of the donor-acceptor pairs in photosynthetic reaction centers via extraction of the dipolar interaction constant [201, 202]. Researchers try to mimic nature and create new artificial systems based on photosynthesis to build-up a biological source of renewable energies based on $\mathrm{CO}_{2}$, also referred to as solar fuel in literature [203]. However, the overall complexity of photosynthesis has not been reproduced so far, as the construction of a concerting relationship between all involved reactions remains a challenge, and still some processes remain unclear.

Quantum coherence is a phenomenon that has been observed in photosystems [204-206], with times of sub- $\mu$ s. More precisely, zero-quantum and double-quantum coherence were measured. Single quantum coherence involves the behavior of one spin, but photoexcitation comes along with formation of radical pairs. Zero-quantum coherence ("quantum beats") describes the process of a mutually-opposed spin flip of a spin pair $\left(M_{S}=0\right)$, while double-quantum coherence describes the spin flip of parallel-oriented spins of pairs $\left(M_{S}= \pm 1\right)$. The observation of quantum beats strongly depends on the environmental spin bath that lead to decoherence [206]. Besides, other biologic matters such as enzymes also show quantum coherence, from which information on magnetic coupling could be extracted [207, 208]. 
(a)
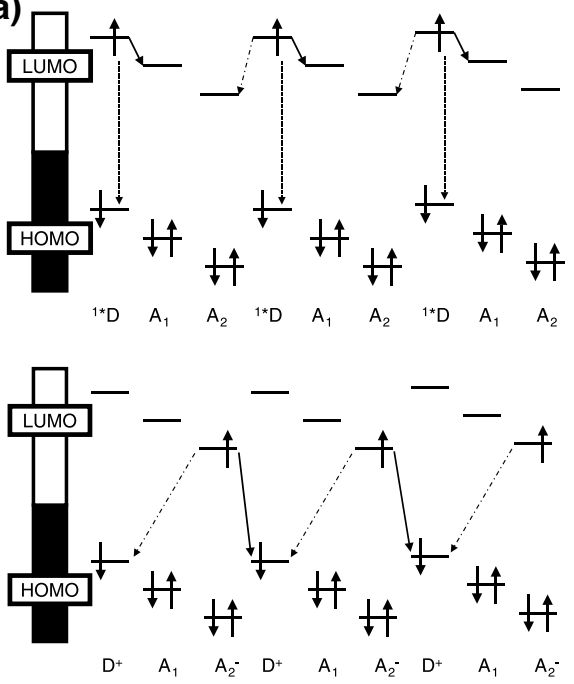

(b)

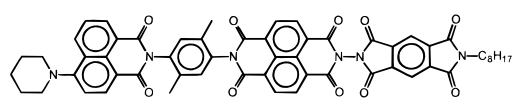

(c)

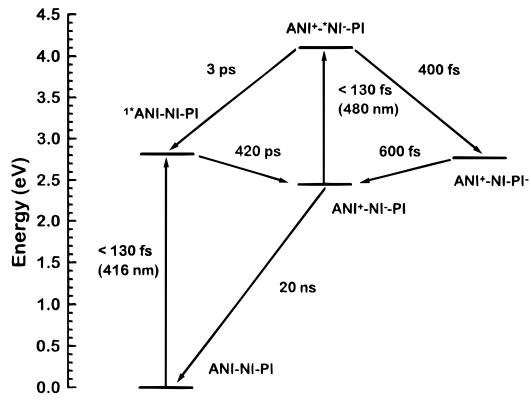

Fig. 34 Molecular register based on donor-acceptor1-acceptor2 molecules $\left(D-A_{1}-A_{2}\right)$. a Principle of a molecular register. The upper image shows the possible deexcitation path after photoexcitation: intramolecular charge transfer to $\mathrm{A}_{1}$ (solid line), charge-transfer to neighboring acceptor $\mathrm{A}_{2}$ (adjacent-dotted line) and fluorescent recombination (dotted line). The lower picture shows the next step: recombination either within the molecule or with adjacent ones. If recombination via QT to neighboring molecules is favored over fluorescence, a charged donor is left at the beginning of the chain, which can be interpreted as a ' 0 '. A ' 1 ' can be achieved via reduction. By repeating the light excitation $n$ times, $n$ bit data can be stored. b Possible D- $\mathrm{A}_{1}-\mathrm{A}_{2}$ molecule for realization of the register. $\mathbf{c}$ Real energy diagram of molecule (b). The scheme for hole transport (a) can be analogously adapted to free electron transport. Pictures adapted from Refs. [209, 211, 212]. Reprinted with permission from Wiley-VCH and Copyright (1996) by the American Chemical Society

\subsubsection{Donor-Acceptor Triads for Molecular Spintronics}

As shown in the last two subsections, spin physics plays an important role in photolysis and in organic solar cells. By understanding their results, similar systems can be uses as molecular switches yielding interesting applications for molecular spintronics [209]. The creation of electron donor-acceptor-based molecular switches (D-A) requires advanced chemical engineering [210].

One of the first theoretical attempts to integrate $\mathrm{D}-\mathrm{A}_{1}-\mathrm{A}_{2}$ systems in molecular electronics was demonstrated in 1988 [211]. Such molecular assemblies can be realized as shift registers (see Fig. 34) thus opening new perspectives for devices, as achieved 8 years later [212]. However, an investigation of spin-spin interactions with TrEPR is made difficult, in this case, by the small charge-transfer time, which lies in the sub-ps regime. The temporal resolution of EPR electronics, however, is around $10 \mathrm{~ns}$.

This scheme motivated the development of donor-bridge-acceptor systems (D-B-A), where a bridge delays recombination and intramolecular electron flows can be studied via EPR. Nonetheless, the bridge can be functionalized enabling 

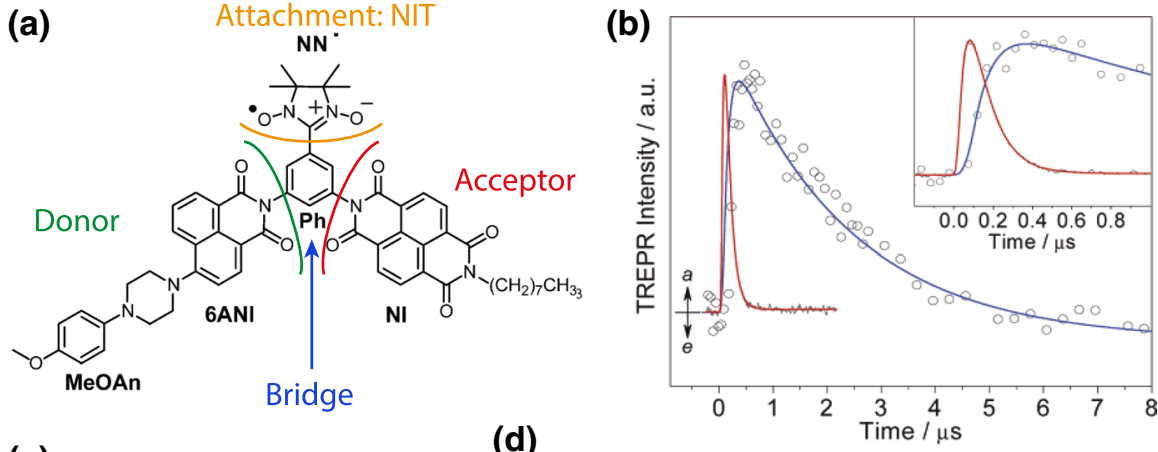

(c)

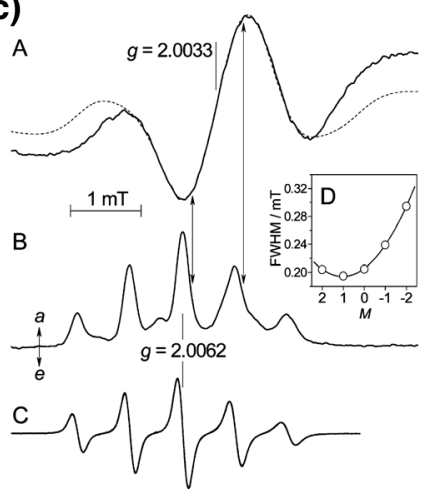

(d)

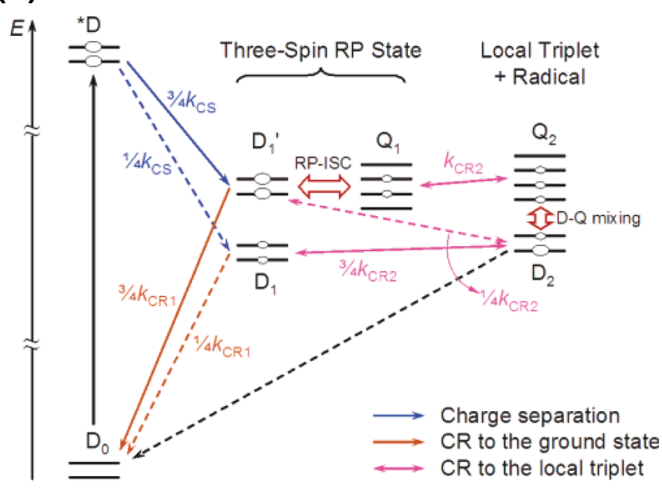

Fig. 35 TrEPR on donor-bridge-acceptor nitronyl-nitroxide (D-B-A-NIT) molecules. a Chemical structure of the D-B-A-NIT complex. Via excitation with $416 \mathrm{~nm}$ light, a radical pair is generated and the electron travels via the bridge-molecule to the acceptor. On its way, it perturbs the attached nitronylnitroxide molecule. b TrEPR results. The signal was acquired after a single $\pi / 2$-pulse (free induction decay, FID). The radical pair signal has been obtained by subtracting the FID for different fields. The blue curve is a pure signature of the NIT-radical, as the radical-pair (RP) signal (red) decays much faster. The excitation of radical pairs is known to be improbable with $\pi / 2$-pulses, but $\pi / 4$-pulses can be used instead [219, 220]. c X-band EPR of D-B-A-NIT in toluene (A) $60 \mathrm{~ns}$ and (B) $320 \mathrm{~ns}$ after laser pulse (absorptive type), and (C) before laser excitation (derivative type). Five lines are observed in absence of light due to hyperfine coupling (D) with two equivalent nitrogen atoms. The evolution shows the distortion generated by the quasi flowing electron. d Energy level diagram of the molecule, resulting from exchange interactions within the triradical (CR: charge recombination). The arrows denote possible transition paths. Figures adapted from Ref. [213]. Reprinted with permission from Copyright (2006) by the American Chemical Society

distinct investigations of interactions with a plethora of attachments. Chernick and co-workers synthesized a D-B-A-NIT-complex, where a nitronyl-nitroxide radical is attached to the bridge (Fig. 35) [213, 214]. The D-B-A building block is based on a work by Lukas et al. and Shaakov et al. [215, 216]. By adding the nitronyl-nitroxide radical, the lifetime of the charge-separated state is enhanced (101 ns vs. $73 \mathrm{~ns}$ ), which the authors attribute to enhanced intersystem crossing (EISC) by the magnetic field of NITs. Similar behavior was observed for TEMPO-attached D-B-A molecules [217, 218]. The interaction pattern leading to the shown fits are not trivial and result from ISC and exchange-interactions 
between the three radicals, yielding doublet and quartet states (Fig. 35d). Interestingly, the nitronyl-nitroxides remain spin-polarized after charge-recombination on a timescale of the radical-pair decay (Fig. 35b), which the authors attribute to spin-selective exchange interaction. Furthermore, the donor-acceptor spin-spin interactions remain unchanged.

A follow-up by Chernick et al. investigated the effect of different attachments to the bridge [221]. The use of BPNO radicals significantly manipulates the relaxation path ways. However, spin polarization on BPNO was not observed due to larger delocalization of the electron on the radical. Via TrEPR, spin dynamics can be tracked, attributed to doublet-quartet transitions [222], and distances be measured [223], a technique commonly used in protein research [124]. Another approach functionalizes either the donor or the acceptor with a radical group, where dynamic spin-polarization has been observed [224]. The rates for charge separation do not change considerably for different attachments to the radical, but the polarization transfer strongly depends on the exchange interaction between the donor and acceptor molecule [225]. While these investigations are valid for quasi-wire-like molecular structures, dipolar interactions become important for folded structures, where lower exchange interaction result in triplet spin dynamics, rather that quartet ones [226].

The theoretical understanding of all these effects is both crucial and challenging. First developments of a toolbox for the investigation of spin dynamics and EISC are under development [227-230]. This becomes particularly important for the observation of unusual effects, e.g. when EISC-induced triplet states play a role in the polarization [231]. Zero-field splitting and quantum decoherence during transfer of population are reported to be one of the key mechanisms for dynamic spin polarization in doublet-quartet systems [230].

New approaches investigate the direct photogeneration of spin-polarized electrons via magnetic donors, and spin-selective acceptors [232]. They overcome the use of spin valves for molecular spintronics as spin-polarized currents are generated intrinsically. Furthermore, control over the lifetime has been achieved using microwave $\pi$-pulses, allowing change of molecular spintronic properties by physical means [233]. To control the properties electronically, D-B-A systems can be functionalized with surface-binding groups [234-237], where basically developed techniques for functionalizing SMMs could be adapted and be used for logic gates [238]. The design of new D-B-A systems is enabled by a plethora of molecular bricks, which are reviewed elsewhere [210, 239-245]. The possibilities D-B-A systems offer are rich and yet wait to be combined with molecular spintronic systems discussed in Sect. 3.

\subsection{Photoswitchable Molecules}

In the last section, we discussed the interactions between photogenerated electrons and spin centers, which lead to spin polarization. In addition to that, properties of molecular magnetic materials can be tuned by external stimuli in general. Beside temperature and magnetic fields, the spin state can be tuned by pressure [246], 

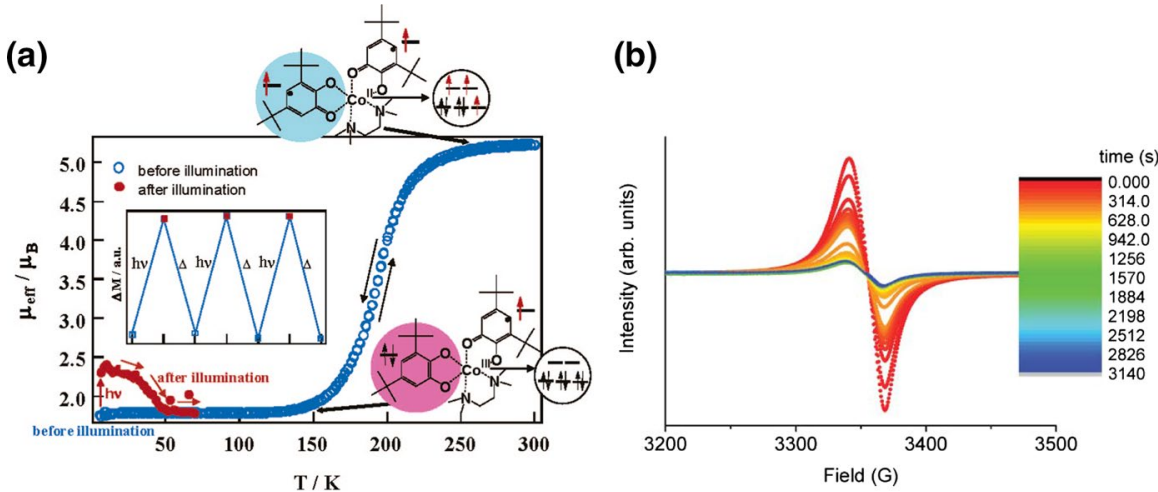

(c)

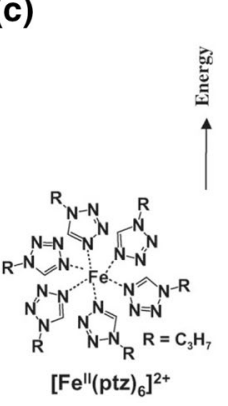

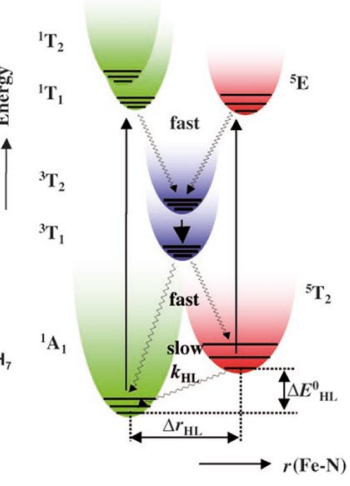

(d)

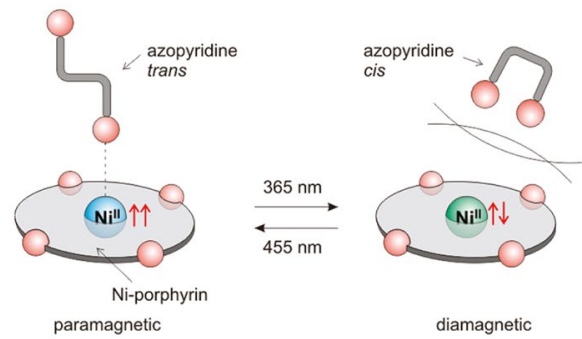

Fig. 36 The three most common processes in photomagnetism. a Valence tautomerism (VT). The graph shows the temperature dependence of the magnetic moment. At room temperature, the VT complex is in a high spin state. It undergoes a transition to a low spin state centered at around $200 \mathrm{~K}$, where a catecholate group is converted to a semiquinone. At low temperatures, the transition can be reversed partially by irradiation with light (red curve). When warming up above $40 \mathrm{~K}$, the system relaxes again to the low spin state. This behavior is due to the fact that via light the system gets trapped in a meta-stable state. To relax, the system needs to overcome an energy barrier either thermally or via quantum tunneling. b EPR signal of the VT complex. The EPR-signal decreases upon irradiation. The light-generated $(\mathrm{SQ})_{2}$ species are reported to be EPR-silent [263, 264]. c Spin-crossover complex. Also here, the system gets trapped in a metastable high-spin state upon light irradiation showing similar magnetic behavior. d Photoisomerization process. Via interaction with different conformations of the azopyride ligand, the nickel ion either forms a triplet or a singlet ground state. This process is totally reversible by changing the irradiation wavelength from 365 to $455 \mathrm{~nm}$, making it an interesting molecular switch. Figures adapted from Refs. [23, 251, 257, 263]. Reprinted with permission from Wiley-VCH, Royal Society of Chemistry and Copyright $(2007,2011)$ by the American Chemical Society

electrical fields [247] and light [23]. As an ultra-clean and fast method, controlling nanomagnets with photons is an appealing feature, which we briefly outline here. Detailed reviews can be found in Ref. [23, 248, 249].

For long-term switching, where any spin state change is maintained for hours, three main concepts have been found so far [29]: valence tautomerism [250-253], spin-crossover [254, 255] and photoisomerization [256, 257]. At room temperature, such systems are usually in a high-spin state, and undergo a low-spin transition or 
even diamagnetic transition at lower temperatures. This process can be switched back using light at low temperatures. Some examples are depicted in Fig. 36. The critical temperatures of such systems usually are below $100 \mathrm{~K}$, except when excitation comes along with reversible reformation of bonds [23]. Such systems could soon be getting interested for room temperature applications. Short-term molecular switches can be realized using electronic transitions in chromium complexes [258], for instance.

Fast electron dynamics is involved in long-term switching [259]. TrEPR studies on $\mathrm{Cu}(\mathrm{hfac})_{2}$-NIT breathing crystals revealed that although the spin conversion is maintained for hours at liquid helium temperatures, self-decelerating spin dynamics, probably due to distribution of relaxation times, at the sub- $\mu$ s drive the spin transitions [260-262].

\section{Conclusion and Perspectives}

Transient EPR represents an informative tool for the investigation of excited spin dynamics, due to the vast amount of information that form the observed spectra, which range from zero-field splitting to metric distances between interacting spins. Decryption of the dynamics still remains a challenging task, especially when intriguing phenomena like dynamic spin polarization are observed the first time. The Hamiltonian of molecular systems can get enormously complex (see Eq. (2)), and photoexcitation just adds another degree of freedom. Luckily, to a certain degree the use of different pulse sequences helps to distinctly select the properties which one wants to investigate [219]. Based on the observations, theoretical models have been developed. Many efforts have been undertaken to explain the spin polarization that occurs in radical-attached triads after photoexcitation, but in our point of view some conclusions originating from anisotropy terms [213] need to be investigated more in detail. Nevertheless, the results on spin dynamics helped to explain phenomena in neighboring disciplines [265].

Up to now, solar cell research and biochemistry mostly benefited from the TrEPR technique. Molecular spintronics on D-B-A triads is still a very fundamental field of research. Research groups around the world mainly focus on how attachments affect the dynamics of the D-B-A systems. However, for a step towards applications in spintronics we propose the opposite direction: how does the charge dynamics in D-B-A systems affect the properties of attachments, either on the bridge or on the acceptor? D-B-A systems provide a basis to investigate charge carrier dynamics not only on radicals, but also on spin centers like SMMs, which has not been tried yet. Furthermore, it is not yet clear how spin-orbit coupling manipulates the behavior of such systems. The studies on the presented radical-based triads give a first hint on how interactions via exchange coupling and dipole-dipole interactions drive such systems. Moreover, the TrEPR technique intrinsically gives access to the measurement of quantum coherence, which is still a difficult task in SMM-based molecular spintronics owing to wavefunction overlaps with electrodes and interactions with the phonon bath, as well as device geometry. SMM-based triads have the potential 
(a)

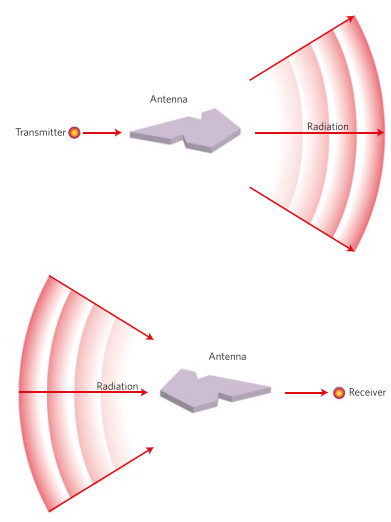

(b)

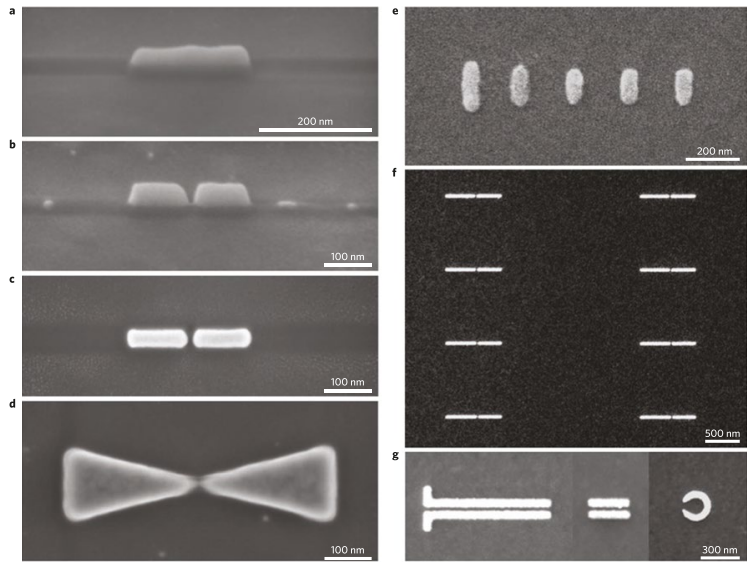

Fig. 37 Nanoantennas as possible building block for molecular spintronics. a Nanoantenna principle. The nanoantenna collects incoming light and direct the energy towards a receiver, and vice versa. b Structures of different kind of nanoantennes generated via electron-beam lithography and subsequent liftoff. Figures adapted from Ref. [266]. Reprinted with permission from Springer Nature

to answer the question how electron flows affect quantum coherence. We discussed how the environmental and chemical changes lead to a strong increase of the coherence time, which is useful for the design of possible SMM-triads that could show quantum coherence. However, a foreseeable coupling of the spin with the D-B-Acomplex, particularly with its corresponding charge-separated state after photoexcitation, might create another decoherence path. Chemical engineering can overcome this issue.

Progress in functionalization enabled sticking of D-B-A molecules on surfaces [234]. However, the photomagnetism community has not benefited much yet from progress in SMM-based molecular spintronics. Integration into nanoelectronic devices is particularly appealing as such triads can act as ultra-clean electron donors, where interactions with electrons can be controlled with light. Bringing light onto such devices could be achieved using nanoantennas which are able to distinctly address single molecules [266, 267]. Nanoantennas, as depicted in Fig. 37, are nanoscopic structures that collect light and transfer the energy to a receiver unit (and vice versa). For instance, they can be used to increase the performance of photovoltaic systems [268]. Together with nanoantennas, radical-attached triads could be an interesting building block for intrinsically generated spin-polarized currents. Furthermore, triads offer the unique feature of well-defined geometry, which means that the same photo-induced electron flow happens on the single-molecule level every time when the triad is being excited by light. Chemical engineering is able to draw on a plethora of donor and acceptor groups to tailor triads towards the needs of molecular spintronic applications, for which the mentioned results from other disciplines are of crucial importance.

An interesting substrate for such molecules are graphene nanoribbons, GNRs. With typical widths in the nanometer range, they represent quasi-one-dimensional systems 
with most types showing a finite bandgap [269, 270], in contrast to gapless two-dimensional graphene flakes [119]. Such GNRs have recently emerged particular interest. Due to advances in fabrication methods, it is now possible to synthesize ultra-clean GNRs via an bottom-up approach [271-273]. This allows us to chemically bond molecules, carrying a spin centre or photoswitchable unit [23, 248, 274, 275], and directly integrate into spintronic devices for which fabrication methods exist [276]. Recently, it has been able to clearly observe the topological edge state in graphene nanoribbons [277], which represented a mainly theoretical concept thus far and experimental hints were scarce until then. The edge state is an additional magnetic state in GNRs with zigzag-type edges [278, 279], and superior spin filtering properties are predicted [280, 281], making them promising materials for future spintronic devices. The method of choice to investigate spin phenomena is EPR. Using advanced techniques, we can decouple the spins from the nuclear spin bath yielding competing quantum coherence times nearly reaching milliseconds, as it has been shown for graphenoids [282, 283]. Addressing single molecules via EPR can then happen using magnetic scanning tunnelling tips [284]. The possibility of chemically grafting molecules allows us with endless possibilities to create an entirely new class of spintronic devices and come up with new theories on the physical properties of graphene nanoribbons.

The possibility of introducing ultra-fast light-switchable elements in molecular spintronic systems on SMM-basis could create a revolution in the field, not only as a new control parameter, but also for performing quantum logic operations.

Funding We thank the European Union (ERC-CoG-773048-MMGNRs) and the Royal Society (URF program) for financial support.

Open Access This article is licensed under a Creative Commons Attribution 4.0 International License, which permits use, sharing, adaptation, distribution and reproduction in any medium or format, as long as you give appropriate credit to the original author(s) and the source, provide a link to the Creative Commons licence, and indicate if changes were made. The images or other third party material in this article are included in the article's Creative Commons licence, unless indicated otherwise in a credit line to the material. If material is not included in the article's Creative Commons licence and your intended use is not permitted by statutory regulation or exceeds the permitted use, you will need to obtain permission directly from the copyright holder. To view a copy of this licence, visit http://creativecommons.org/licen ses/by/4.0/.

\section{References}

1. I.L. Markov, Nature 512, 147 (2014)

2. S. Gao (ed.), Molecular Nanomagnets and Related Phenomena (Springer Verlag, Berlin, Heidelberg, 2014)

3. L. Bogani, W. Wernsdorfer, Nat. Mater. 7, 179 (2008)

4. H.B. Heersche, Z. de Groot, J.A. Folk, H.S.J. van der Zant, C. Romeike, M.R. Wegewijs, L. Zobbi, D. Barreca, E. Tondello, A. Cornia, Phys. Rev. Lett. 96, 206801 (2006)

5. J.A. Mol, C.S. Lau, W.J.M. Lewis, H. Sadeghi, C. Roche, A. Cnossen, J.H. Warner, C.J. Lambert, H.L. Anderson, G.A.D. Briggs, Nanoscale 7, 13181 (2015)

6. S.Y. Quek, M. Kamenetska, M.L. Steigerwald, H.J. Choi, S.G. Louie, M.S. Hybertsen, J.B. Neaton, L. Venkataraman, Nat. Nanotech. 4, 230 (2009)

7. R. Vincent, S. Klyatskaya, M. Ruben, W. Wernsdorfer, F. Balestro, Nature 488, 357 (2012) 
8. D. Gatteschi, R. Sessoli, J. Villain, Molecular Nanomagnets (Oxford University Press, Oxford, New York, 2006)

9. J.R. Friedman, M.P. Sarachik, Annu. Rev. Condens. Matter Phys. 1(1), 109 (2010)

10. M.N. Leuenberger, D. Loss, Nature 410, 789 (2001)

11. J. van Slageren, Top. Curr. Chem. 321, 199 (2012)

12. M. del Carmen Giménez-López, F. Moro, A.L. Torre, C.J. Gómez-García, P.D. Brown, J. van Slageren, A.N. Khlobystov, Nat. Commun. 2, 407 (2011)

13. J.M.D. Coey, Magnetism and Magnetic Materials (Cambridge University Press, Cambridge, New York, 2010)

14. L.H. Reddy, J.L. Arias, J. Nicolas, P. Couvreur, Chem. Rev. 112(11), 5818 (2012)

15. A.H. Lu, E.L. Salabas, F. Schüth, Angew. Chem. Int. Ed. 46(8), 1222 (2007)

16. R. Sessoli, H.L. Tsai, A.R. Schake, S. Wang, J.B. Vincent, K. Folting, D. Gatteschi, G. Christou, D.N. Hendrickson, J. Am. Chem. Soc. 115(5), 1804 (1993)

17. A.J. Tasiopoulos, A. Vinslava, W. Wernsdorfer, K.A. Abboud, G. Christou, Angew. Chem. Int. Ed. 43(16), 2117 (2004)

18. S. Accorsi, A.L. Barra, A. Caneschi, G. Chastanet, A. Cornia, A.C. Fabretti, D. Gatteschi, C. Mortalò, E. Olivieri, F. Parenti, P. Rosa, R. Sessoli, L. Sorace, W. Wernsdorfer, L. Zobbi, J. Am. Chem. Soc. 128(14), 4742 (2006)

19. D.N. Woodruff, R.E.P. Winpenny, R.A. Layfield, Chem. Rev. 113(7), 5110 (2013)

20. N. Ishikawa, M. Sugita, T. Ishikawa, S. Ya Koshihara, Y. Kaizu, J. Am. Chem. Soc. 125(29), 8694 (2003)

21. E. Heintze, F. el Hallak, C. Clauß, A. Rettori, M.G. Pini, F. Totti, M. Dressel, L. Bogani, Nat. Mater. 12, 202 (2013)

22. R. Sessoli, D. Gatteschi, A. Caneschi, M.A. Novak, Nature 365, 141 (1993)

23. O. Sato, J. Tao, Y.Z. Zhang, Angew. Chem. Int. Ed. 46(13), 2152 (2007)

24. X. Feng, C. Mathonière, I.R. Jeon, M. Rouzières, A. Ozarowski, M.L. Aubrey, M.I. Gonzalez, R. Clérac, J.R. Long, J. Am. Chem. Soc. 135(42), 15880 (2013)

25. R. Bircher, G. Chaboussant, C. Dobe, H.U. Güdel, S.T. Ochsenbein, A. Sieber, O. Waldmann, Adv. Funct. Mater. 16(2), 209 (2006)

26. A. Sieber, D. Foguet-Albiol, O. Waldmann, S.T. Ochsenbein, G. Carver, H. Mutka, F. FernandezAlonso, M. Mezouar, H.P. Weber, G. Christou, H.U. Güdel, Phys. Rev. B 74, 024405 (2006)

27. A.S. Zyazin, J.W.G. van den Berg, E.A. Osorio, H.S.J. van der Zant, N.P. Konstantinidis, M. Leijnse, M.R. Wegewijs, F. May, W. Hofstetter, C. Danieli, A. Cornia, Nano Lett. 10(9), 3307 (2010)

28. J. Hu, R. Wu, Phys. Rev. Lett. 110, 097202 (2013)

29. C. Cervetti, E. Heintze, L. Bogani, Dalton Trans. 43, 4220 (2014)

30. M. Mannini, F. Pineider, P. Sainctavit, L. Joly, A. Fraile-Rodríguez, M.-A. Arrio, C.C. dit Moulin, W. Wernsdorfer, A. Cornia, D. Gatteschi, R. Sessoli, Adv. Mater. 21(2), 167 (2009)

31. R.J. Holmberg, M. Murugesu, J. Mater. Chem. C 3, 11986 (2015)

32. N. Domingo, E. Bellido, D. Ruiz-Molina, Chem. Soc. Rev. 41, 258 (2012)

33. D. Gatteschi, A. Cornia, M. Mannini, R. Sessoli, Inorg. Chem. 48(8), 3408 (2009)

34. S. Goswami, A.K. Mondal, S. Konar, Inorg. Chem. Front. 2, 687 (2015)

35. E. Moreno Pineda, T. Komeda, K. Katoh, M. Yamashita, M. Ruben, Dalton Trans. 45, 18417 (2016)

36. A. Cornia, M. Mannini, R. Sessoli, D. Gatteschi, Eur. J. Inorg. Chem. 2019(5), 552 (2019)

37. A. Caneschi, D. Gatteschi, R. Sessoli, A.L. Barra, L.C. Brunel, M. Guillot, J. Am. Chem. Soc. 113(15), 5873 (1991)

38. J.R. Friedman, M.P. Sarachik, J. Tejada, R. Ziolo, Phys. Rev. Lett. 76, 3830 (1996)

39. A.M. Ako, I.J. Hewitt, V. Mereacre, R. Clérac, W. Wernsdorfer, C.E. Anson, A.K. Powell, Angew. Chem. Int. Ed. 45(30), 4926 (2006)

40. L. Sorace, C. Benelli, D. Gatteschi, Chem. Soc. Rev. 40, 3092 (2011)

41. J.D. Rinehart, J.R. Long, Chem. Sci. 2, 2078 (2011)

42. H.L. Feltham, S. Brooker, Coord. Chem. Rev. 276, 1 (2014)

43. R.A. Layfield, Organometallics 33(5), 1084 (2014)

44. G.A. Craig, M. Murrie, Chem. Soc. Rev. 44, 2135 (2015)

45. J.M. Frost, K.L.M. Harriman, M. Murugesu, Chem. Sci. 7, 2470 (2016)

46. J.D. Rinehart, M. Fang, W.J. Evans, J.R. Long, J. Am. Chem. Soc. 133(36), 14236 (2011)

47. C.A.P. Goodwin, F. Ortu, D. Reta, N.F. Chilton, D.P. Mills, Nature 548(7668), 439 (2017) 
48. F.S. Guo, B.M. Day, Y.C. Chen, M.L. Tong, A. Mansikkamäki, R.A. Layfield, Science 362(6421), 1400 (2018)

49. M.J. Giansiracusa, A.K. Kostopoulos, D. Collison, R.E.P. Winpenny, N.F. Chilton, Chem. Commun. 55, 7025 (2019)

50. S.T. Liddle, J. van Slageren, Chem. Soc. Rev. 44, 6655 (2015)

51. C. Görller-Walrand, K. Binnemans, Handb. Phys. Chem. Rare Earths 23, 121 (1996)

52. G. Cucinotta, M. Perfetti, J. Luzon, M. Etienne, P.E. Car, A. Caneschi, G. Calvez, K. Bernot, R. Sessoli, Angew. Chem. Int. Ed. 51(7), 1606 (2012)

53. M.E. Boulon, G. Cucinotta, J. Luzon, C. Degl'Innocenti, M. Perfetti, K. Bernot, G. Calvez, A. Caneschi, R. Sessoli, Angew. Chem. Int. Ed. 52(1), 350 (2013)

54. A. Abragam, B. Bleaney, Electron Paramagnetic Resonance of Transition Ions (Oxford University Press, Oxford, 2012)

55. H. Lueken, Magnetochemie (Teubner, Stuttgart, 1999)

56. N.W. Ashcroft, N.D. Mermin, in Solid State Physics (Cengage Learning. Inc, 1976)

57. I. Dzyaloshinsky, J. Phys. Chem. Solids 4(4), 241 (1958)

58. T. Moriya, Phys. Rev. 120, 91 (1960)

59. M. Slota, S.D. Jiang, E. Heintze, Y. Rechkemmer, M. Dressel, J. van Slageren, L. Bogani, Phys. Rev. B 99, 134410 (2019)

60. M.N. Baibich, J.M. Broto, A. Fert, F.N. Van Dau, F. Petroff, P. Etienne, G. Creuzet, A. Friederich, J. Chazelas, Phys. Rev. Lett. 61, 2472 (1988)

61. G. Binasch, P. Grünberg, F. Saurenbach, W. Zinn, Phys. Rev. B 39, 4828 (1989)

62. M.A. Ruderman, C. Kittel, Phys. Rev. 96, 99 (1954)

63. T. Kasuya, Prog. Theor. Phys. 16(1), 45 (1956)

64. K. Yosida, Phys. Rev. 106, 893 (1957)

65. N.F. Mott, Proc. R. Soc. A 880, 699 (1936)

66. H.J. Richter, J. Phys. D Appl. Phys. 40(9), R149 (2007)

67. R. Wood, J. Magn. Magn. Mater. 321(6), 555 (2009)

68. S. Mao, Y. Chen, F. Liu, X. Chen, B. Xu, P. Lu, M. Patwari, H. Xi, C. Chang, B. Miller, D. Menard, B. Pant, J. Loven, K. Duxstad, S. Li, Z. Zhang, A. Johnston, R. Lamberton, M. Gubbins, T. McLaughlin, J. Gadbois, J. Ding, B. Cross, S. Xue, P. Ryan, IEEE Trans. Magn. 42(2), 97 (2006)

69. T. Kagami, T. Kuwashima, S. Miura, T. Uesugi, K. Barada, N. Ohta, N. Kasahara, K. Sato, T. Kanaya, H. Kiyono, N. Hachisuka, S. Saruki, K. Inage, N. Takahashi, K. Terunuma, IEEE Trans. Magn. 42(2), 93 (2006)

70. M. Julliere, Phys. Lett. A 54(3), 225 (1975)

71. T. Miyazaki, N. Tezuka, J. Magn. Magn. Mater. 151(3), 403 (1995)

72. D. Wang, C. Nordman, J. Daughton, Z. Qian, J. Fink, IEEE T. Magn. 40(4), 2269 (2004)

73. S.S.P. Parkin, C. Kaiser, A. Panchula, P.M. Rice, B. Hughes, M. Samant, S.H. Yang, Nat. Mater. 3, 862 (2004)

74. S. Yuasa, T. Nagahama, A. Fukushima, Y. Suzuki, K. Ando, Nat. Mater. 3, 868 (2004)

75. S. Ikeda, J. Hayakawa, Y. Ashizawa, Y.M. Lee, K. Miura, H. Hasegawa, M. Tsunoda, F. Matsukura, H. Ohno, Appl. Phys. Lett. 93(8), 082508 (2008)

76. S. Sanvito, Chem. Soc. Rev. 40, 3336 (2011)

77. K.V. Raman, App. Phys. Rev. 1(3), 031101 (2014)

78. A. Cornia, P. Seneor, Nat. Mater. 16(5), 505 (2017)

79. A. Bellec, J. Lagoute, V. Repain, C. R. Chim. 21(12), 1287 (2018)

80. M. Gobbi, M.A. Novak, E. Del Barco, J. Appl. Phys. 125(24), 240401 (2019)

81. M. Cinchetti, V.A. Dediu, L.E. Hueso, Nat. Mater. 16(5), 507 (2017)

82. A. Ghirri, A. Candini, M. Affronte, Magnetochem. 3, 1 (2017)

83. M. Shiddiq, D. Komijani, Y. Duan, A. Gaita-Ariño, E. Coronado, S. Hill, Nature 531(7594), 348 (2016)

84. E. Coronado, Nat. Rev. Mater. 5(2), 87 (2020)

85. S. Jiang, K. Goß, C. Cervetti, L. Bogani, Sci. China Chem. 55(6), 867 (2012)

86. L. Bogani, in Molecular Nanomagnets and Related Phenomena, ed. by S. Gao (Springer Verlag, Berlin, Heidelberg, 2014), pp. 331-382

87. M.A. Kastner, Phys. Today 24, 24 (1993)

88. S. Voss, M. Burgert, M. Fonin, U. Groth, U. Rüdiger, Dalton Trans. 2008, 499-505 (2008) 
89. M. Mannini, P. Sainctavit, R. Sessoli, C.C. dit Moulin, F. Pineider, M.A. Arrio, A. Cornia, D. Gatteschi, Chem. Eur. J. 14(25), 7530 (2008)

90. S. Kahle, Z. Deng, N. Malinowski, C. Tonnoir, A. Forment-Aliaga, N. Thontasen, G. Rinke, D. Le, V. Turkowski, T.S. Rahman, S. Rauschenbach, M. Ternes, K. Kern, Nano Lett. 12(1), 518 (2012)

91. M. Mannini, F. Pineider, P. Sainctavit, C. Danieli, E. Otero, C. Sciancalepore, A.M. Talarico, M.A. Arrio, A. Cornia, D. Gatteschi, R. Sessoli, Nat. Mater. 8, 194 (2009)

92. Y.-H. Zhang, S. Kahle, T. Herden, C. Stroh, M. Mayor, U. Schlickum, M. Ternes, P. Wahl, K. Kern, Nat. Commun. 4, 2110 (2013)

93. C. Wäckerlin, F. Donati, A. Singha, R. Baltic, S. Rusponi, K. Diller, F. Patthey, M. Pivetta, Y. Lan, S. Klyatskaya, M. Ruben, H. Brune, J. Dreiser, Adv. Mater. 28(26), 5195 (2016)

94. A. Cornia, M. Mannini, P. Sainctavit, R. Sessoli, Chem. Soc. Rev. 40, 3076 (2011)

95. J.V. Barth, G. Costantini, K. Kern, Nature 437, 671 (2005)

96. T. Komeda, H. Isshiki, J. Liu, Y.F. Zhang, N. Lorente, K. Katoh, B.K. Breedlove, M. Yamashita, Nat. Commun. 2, 217 (2011)

97. T. Miyamachi, M. Gruber, V. Davesne, M. Bowen, S. Boukari, L. Joly, F. Scheurer, G. Rogez, T.K. Yamada, P. Ohresser, E. Beaurepaire, W. Wulfhekel, Nat. Commun. 3, 938 (2012)

98. S. Javaid, S. Lebègue, B. Detlefs, F. Ibrahim, F. Djeghloul, M. Bowen, S. Boukari, T. Miyamachi, J. Arabski, D. Spor, J. Zegenhagen, W. Wulfhekel, W. Weber, E. Beaurepaire, M. Alouani, Phys. Rev. B 87, 155418 (2013)

99. K. Katoh, Y. Yoshida, M. Yamashita, H. Miyasaka, B.K. Breedlove, T. Kajiwara, S. Takaishi, N. Ishikawa, H. Isshiki, Y.F. Zhang, T. Komeda, M. Yamagishi, J. Takeya, J. Am. Chem. Soc. 131(29), 9967 (2009)

100. S. Müllegger, S. Tebi, A.K. Das, W. Schöfberger, F. Faschinger, R. Koch, Phys. Rev. Lett. 113, 133001 (2014)

101. M.H. Jo, J.E. Grose, K. Baheti, M.M. Deshmukh, J.J. Sokol, E.M. Rumberger, D.N. Hendrickson, J.R. Long, H. Park, D.C. Ralph, Nano Lett. 6(9), 2014 (2006)

102. J.J. Parks, A.R. Champagne, T.A. Costi, W.W. Shum, A.N. Pasupathy, E. Neuscamman, S. FloresTorres, P.S. Cornaglia, A.A. Aligia, C.A. Balseiro, G.K.L. Chan, H.D. Abruña, D.C. Ralph, Science 328(5984), 1370 (2010)

103. S. Wagner, F. Kisslinger, S. Ballmann, F. Schramm, R. Chandrasekar, T. Bodenstein, O. Fuhr, D. Secker, K. Fink, M. Ruben, H.B. Weber, Nat. Nanotech. 8, 575 (2013)

104. S. Thiele, F. Balestro, R. Ballou, S. Klyatskaya, M. Ruben, W. Wernsdorfer, Science 344(6188), 1135 (2014)

105. W. Liang, M.P. Shores, M. Bockrath, J.R. Long, H. Park, Nature 417, 725 (2002)

106. L. Bogani, C. Danieli, E. Biavardi, N. Bendiab, A.L. Barra, E. Dalcanale, W. Wernsdorfer, A. Cornia, Angew. Chem. Int. Ed. 121(4), 760 (2009)

107. R.J. Chen, Y. Zhang, D. Wang, H. Dai, J. Am. Chem. Soc. 123(16), 3838 (2001)

108. L. Bogani, R. Maurand, L. Marty, C. Sangregorio, C. Altavilla, W. Wernsdorfer, J. Mater. Chem. 20, 2099 (2010)

109. A. Hirsch, Angew. Chem. Int. Ed. 41(11), 1853 (2002)

110. W. Kim, A. Javey, O. Vermesh, Q. Wang, Y.L.H. Dai, Nano Lett. 3(2), 193 (2003)

111. S. Datta, L. Marty, J.P. Cleuziou, C. Tilmaciu, B. Soula, E. Flahaut, W. Wernsdorfer, Phys. Rev. Lett. 107, 186804 (2011)

112. J.P. Cleuziou, W. Wernsdorfer, V. Bouchiat, T. Ondarçuhu, M. Monthioux, Nat. Nanotech. 1, 53 (2006)

113. M. Urdampilleta, S. Klyatskaya, J.P. Cleuziou, M. Ruben, W. Wernsdorfer, Nat. Mater. 10, 502 (2011)

114. M. Ganzhorn, S. Klyatskaya, M. Ruben, W. Wernsdorfer, Nat. Nanotech. 8, 165 (2013)

115. A. Giusti, G. Charron, S. Mazerat, J.D. Compain, P. Mialane, A. Dolbecq, E. Rivière, W. Wernsdorfer, R.N. Biboum, B. Keita, L. Nadjo, A. Filoramo, J.P. Bourgoin, T. Mallah, Angew. Chem. Int. Ed. 48(27), 4949 (2009)

116. S. Sapmaz, P. Jarillo-Herrero, Y.M. Blanter, C. Dekker, H.S.J. van der Zant, Phys. Rev. Lett. 96, 026801 (2006)

117. A. Candini, S. Klyatskaya, M. Ruben, W. Wernsdorfer, M. Affronte, Nano Lett. 11(7), 2634 (2011)

118. D.P. DiVincenzo, E.J. Mele, Phys. Rev. B 29, 1685 (1984)

119. A.K. Geim, K.S. Novoselov, Nat. Mater. 6, 183 (2007)

120. M. Studniarek, C. Wäckerlin, A. Singha, R. Baltic, K. Diller, F. Donati, S. Rusponi, H. Brune, Y. Lan, S. Klyatskaya, M. Ruben, A.P. Seitsonen, J. Dreiser, Adv. Sci. 6(22), 1901736 (2019) 
121. G. Jeschke, in ESR Spectroscopy in Membrane Biophysics, ed. by M.A. Hemminga, L.J. Berliner (Springer Verlag US, New York, 2007), pp. 17-47

122. J.A. Weil, J.R. Bolton (eds.), Electron Paramagnetic Resonance: Elementary Theory and Practical Applications (Wiley, Hoboken, New Jersey, 2007)

123. G.R. Eaton, S.S. Eaton, D.P. Barr, R.T. Weber, Quantitative EPR (Springer Verlag Wien, New York, 2010)

124. A. Schweiger, G. Jeschke, Principles of Pulse Electron Paramagnetic Resonance (Oxford University Press, Oxford, New York, 2001)

125. M.A. Nielsen, I.L. Chuang, Quantum Computation and Quantum Information (Cambridge University Press, Cambridge, New York, 2000)

126. N.V. Prokof'ev, P.C.E. Stamp, Rep. Prog. Phys. 63(4), 669 (2000)

127. A. Morello, P.C.E. Stamp, I.S. Tupitsyn, Phys. Rev. Lett. 97, 207206 (2006)

128. D. Loss, D.P. DiVincenzo, Phys. Rev. A 57, 120 (1998)

129. J.R. Petta, A.C. Johnson, J.M. Taylor, E.A. Laird, A. Yacoby, M.D. Lukin, C.M. Marcus, M.P. Hanson, A.C. Gossard, Science 309(5744), 2180 (2005)

130. J.M. Elzerman, R. Hanson, L.H.W. van Beveren, S. Tarucha, L.M.K. Vandersypen, L.P. Kouwenhoven, Lect. Notes Phys. 667, 25 (2005)

131. J. Clarke, F.K. Wilhelm, Nature 453, 1031 (2008)

132. R. Blatt, D. Wineland, Nature 453, 1008 (2008)

133. L. Childress, R. Hanson, MRS Bull. 38, 134 (2013)

134. J. Wrachtrup, S.Y. Kilin, A.P. Nizovtsev, Opt. Spectrosc. 91(3), 429 (2001)

135. C. Görller-Walrand, K. Binnemans, Handb. Phys. Chem. Rare Earths 25, 101 (1998)

136. P. Atkins, R. Friedman, Molecular Quantum Mechanics (Oxford University Press, Oxford, 2005)

137. E.L. Hahn, Phys. Rev. 80, 580 (1950)

138. S.S. Eaton, G.R. Eaton, L.J. Berliner (eds.), Biomedical EPR - Part A: Free Radicals, Metals, Medicine and Physiology, vol. 24 (Springer, US, New York, 2005)

139. F.K. Larsen, E.J.L. McInnes, H.E. Mkami, J. Overgaard, S. Piligkos, G. Rajaraman, E. Rentschler, A.A. Smith, G.M. Smith, V. Boote, M. Jennings, G.A. Timco, R.E.P. Winpenny, Angew. Chem. Int. Ed. 42(1), 101 (2003)

140. A. Ardavan, O. Rival, J.J.L. Morton, S.J. Blundell, A.M. Tyryshkin, G.A. Timco, R.E.P. Winpenny, Phys. Rev. Lett. 98, 057201 (2007)

141. T. Prisner, 7th EFEPR Summer School Lecture Notes (2015)

142. J.J. Yin, J.S. Hyde, Z. Phys. Chem. (N. F.) 153, 57 (1987)

143. J.S. Hyde, in Time Domain Electron Spin Resonance, ed. by L. Kevan, R.N. Schwartz (WileyInterscience, New York, 1979), p. 1

144. J.C. Gill, J. Phys. C: Solid State Phys. 6(1), 109 (1973)

145. K.N. Shrivastava, Phys. Status Solidi B 117(2), 437 (1983)

146. C. Schlegel, J. van Slageren, G. Timco, R.E.P. Winpenny, M. Dressel, Phys. Rev. B 83, 134407 (2011)

147. G. Mitrikas, Y. Sanakis, C.P. Raptopoulou, G. Kordas, G. Papavassiliou, Phys. Chem. Chem. Phys. 10, $743(2008)$

148. S. Bahr, K. Petukhov, V. Mosser, W. Wernsdorfer, Phys. Rev. Lett. 99, 147205 (2007)

149. M. Bal, J.R. Friedman, W. Chen, M.T. Tuominen, C.C. Beedle, E.M. Rumberger, D.N. Hendrickson, EPL-Europhys. Lett. 82(1), 17005 (2008)

150. S. Takahashi, J. van Tol, C.C. Beedle, D.N. Hendrickson, L.C. Brunel, M.S. Sherwin, Phys. Rev. Lett. 102, 087603 (2009)

151. F. Luis, F.L. Mettes, J. Tejada, D. Gatteschi, L.J. de Jongh, Phys. Rev. Lett. 85, 4377 (2000)

152. S. Hill, R.S. Edwards, N. Aliaga-Alcalde, G. Christou, Science 302(5647), 1015 (2003)

153. S. Carretta, P. Santini, G. Amoretti, T. Guidi, J.R.D. Copley, Y. Qiu, R. Caciuffo, G. Timco, R.E.P. Winpenny, Phys. Rev. Lett. 98, 167401 (2007)

154. C. Schlegel, J. van Slageren, M. Manoli, E.K. Brechin, M. Dressel, Phys. Rev. Lett. 101, 147203 (2008)

155. S. Bertaina, S. Gambarelli, T. Mitra, B. Tsukerblat, A. Müller, B. Barbara, Nature 453, 203 (2008)

156. C.J. Wedge, G.A. Timco, E.T. Spielberg, R.E. George, F. Tuna, S. Rigby, E.J.L. McInnes, R.E.P. Winpenny, S.J. Blundell, A. Ardavan, Phys. Rev. Lett. 108, 107204 (2012)

157. D. Kaminski, A.L. Webber, C.J. Wedge, J. Liu, G.A. Timco, I.N.J. Vitorica-Yrezabal, E.J.L. McInnes, R.E.P. Winpenny, A. Ardavan, Phys. Rev. B 90, 184419 (2014) 
158. M.J. Martínez-Pérez, S. Cardona-Serra, C. Schlegel, F. Moro, P.J. Alonso, H. Prima-García, J.M. Clemente-Juan, M. Evangelisti, A. Gaita-Ariño, J. Sesé, J. van Slageren, E. Coronado, F. Luis, Phys. Rev. Lett. 108, 247213 (2012)

159. W.B. Mims, Phys. Rev. B 5, 2409 (1972)

160. L.G. Rowan, E.L. Hahn, W.B. Mims, Phys. Rev. 137, A61 (1965)

161. F. Moro, D. Kaminski, F. Tuna, G.F.S. Whitehead, G.A. Timco, D. Collison, R.E.P. Winpenny, A. Ardavan, E.J.L. McInnes, Chem. Commun. 50, 91 (2014)

162. S. Bertaina, S. Gambarelli, T. Mitra, B. Tsukerblat, A. Müller, B. Barbara, Nature 466, 1006 (2010)

163. J.M. Zadrozny, J. Niklas, O.G. Poluektov, D.E. Freedman, ACS Cent. Sci. 1(9), 488 (2015)

164. C.J. Yu, M.J. Graham, J.M. Zadrozny, J. Niklas, M.D. Krzyaniak, M.R. Wasielewski, O.G. Poluektov, D.E. Freedman, J. Am. Chem. Soc. 138(44), 14678 (2016)

165. M.J. Graham, C.J. Yu, M.D. Krzyaniak, M.R. Wasielewski, D.E. Freedman, J. Am. Chem. Soc. 139(8), 3196 (2017)

166. M.J. Graham, M.D. Krzyaniak, M.R. Wasielewski, D.E. Freedman, Inorg. Chem. 56(14), 8106 (2017)

167. L. Tesi, E. Lucaccini, I. Cimatti, M. Perfetti, M. Mannini, M. Atzori, E. Morra, M. Chiesa, A. Caneschi, L. Sorace, R. Sessoli, Chem. Sci. 7, 2074 (2016)

168. M. Atzori, L. Tesi, S. Benci, A. Lunghi, R. Righini, A. Taschin, R. Torre, L. Sorace, R. Sessoli, J. Am. Chem. Soc. 139(12), 4338 (2017)

169. L. Tesi, A. Lunghi, M. Atzori, E. Lucaccini, L. Sorace, F. Totti, R. Sessoli, Dalton Trans. 45, 16635 (2016)

170. M. Atzori, E. Morra, L. Tesi, A. Albino, M. Chiesa, L. Sorace, R. Sessoli, J. Am. Chem. Soc. 138(35), 11234 (2016)

171. A. Albino, S. Benci, L. Tesi, M. Atzori, R. Torre, S. Sanvito, R. Sessoli, A. Lunghi, Inorg. Chem. 58(15), 10260 (2019)

172. M.J. Graham, J.M. Zadrozny, M.S. Fataftah, D.E. Freedman, Chem. Mater. 29(5), 1885 (2017)

173. M. Atzori, A. Chiesa, E. Morra, M. Chiesa, L. Sorace, S. Carretta, R. Sessoli, Chem. Sci. 9, 6183 (2018)

174. C. Bonizzoni, A. Ghirri, M. Atzori, L. Sorace, R. Sessoli, M. Affronte, Sci. Rep. 7(1), 13096 (2017)

175. M.J. Graham, J.M. Zadrozny, M. Shiddiq, J.S. Anderson, M.S. Fataftah, S. Hill, D.E. Freedman, J. Am. Chem. Soc. 136(21), 7623 (2014)

176. R.H. Ruby, I.D. Kuntz, M. Calvin, Proc. Natl. Acad. Sci. USA 51(3), 515 (1964)

177. M. Calvin, G.M. Androes, Science 138(3543), 867 (1962)

178. S.S. Kim, S.I. Weissman, J. Magn. Reson. (1969) 24(1), 167 (1976)

179. R. Bittl, S. Weber, Biochim. Biophys. Acta Bioenerg. 1707(1), 117 (2005)

180. S. Weber, C.W.M. Kay, H. Mögling, K. Möbius, K. Hitomi, T. Todo, Proc. Natl. Acad. Sci. U.S.A. 99(3), 1319 (2002)

181. J. Behrends, A. Sperlich, A. Schnegg, T. Biskup, C. Teutloff, K. Lips, V. Dyakonov, R. Bittl, Phys. Rev. B 85, 125206 (2012)

182. F. Kraffert, R. Steyrleuthner, S. Albrecht, D. Neher, M.C. Scharber, R. Bittl, J. Behrends, J. Phys. Chem. C 118(49), 28482 (2014)

183. A. Rao, P.C.Y. Chow, S. Gelinas, C.W. Schlenker, C.Z. Li, H.L. Yip, A.K.Y. Jen, D.S. Ginger, R.H. Friend, Nature 500, 435 (2013)

184. T.A. Ford, I. Avilov, D. Beljonne, N.C. Greenham, Phys. Rev. B 71, 125212 (2005)

185. T. Offermans, P.A. van Hal, S.C.J. Meskers, M.M. Koetse, R.A.J. Janssen, Phys. Rev. B 72, 045213 (2005)

186. L. Franco, A. Toffoletti, M. Ruzzi, L. Montanari, C. Carati, L. Bonoldi, R. Po, J. Phys. Chem. C 117(4), 1554 (2013)

187. A.J. Hoff (ed.), in Advanced EPR: Applications in Biology and Biochemistry (Elsevier, Amsterdam, New York, 1989)

188. S. Stoll, A. Schweiger, J. Magn. Reson. 178(1), 42 (2006)

189. V. Lang, C.C. Lo, R.E. George, S.A. Lyon, J. Bokor, T. Schenkel, A. Ardavan, J.J.L. Morton, Rev. Sci. Instrum. 82(3), 034704 (2011)

190. D.J. Lepine, Phys. Rev. B 6, 436 (1972)

191. D.R. McCamey, H. Huebl, M.S. Brandt, W.D. Hutchison, J.C. McCallum, R.G. Clark, A.R. Hamilton, Appl. Phys. Lett. 89(18), 182115 (2006)

192. C. Boehme, K. Lips, Phys. B: Condens. Matter 376-377, 930 (2006) 
193. H. Huebl, R.P. Starrett, D.R. McCamey, A.J. Ferguson, L.H. Willems van Beveren, Rev. Sci. Instrum. 80(11), 114705 (2009)

194. A. Schnegg, J. Behrends, M. Fehr, K. Lips, Phys. Chem. Chem. Phys. 14, 14418 (2012)

195. H. Huebl, F. Hoehne, B. Grolik, A.R. Stegner, M. Stutzmann, M.S. Brandt, Phys. Rev. Lett. 100, $177602(2008)$

196. F. Kraffert, R. Steyrleuthner, C. Meier, R. Bittl, J. Behrends, Appl. Phys. Lett. 107(4), 043302 (2015)

197. D. Mühlbacher, M. Scharber, M. Morana, Z. Zhu, D. Waller, R. Gaudiana, C. Brabec, Adv. Mater. 18(21), 2884 (2006)

198. J. Barber, Cold Spring Harb. Symp. Quant. Biol. 77, 295 (2012)

199. G. Renger, Biochim. Biophys. Acta Bioenerg. 1503(1-2), 210 (2001)

200. M. Kammel, J. Kern, W. Lubitz, R. Bittl, Biochim. Biophys. Acta Bioenerg. 1605(1-3), 47 (2003)

201. Y. Kodera, K. Takura, A. Kawamori, Biochim. Biophys. Acta Bioenerg. 1101(1), 23 (1992)

202. S.G. Zech, W. Lubitz, R. Bittl, Ber. Bunsenges. Phys. Chem. 100(12), 2041 (1996)

203. S. Berardi, S. Drouet, L. Francas, C. Gimbert-Suriñach, M. Guttentag, C. Richmond, T. Stoll, A. Llobet, Chem. Soc. Rev. 43, 7501 (2014)

204. G. Kothe, S. Weber, E. Ohmes, M.C. Thurnauer, J.R. Norris, J. Phys. Chem. 98(10), 2706 (1994)

205. A.J. Hoff, P. Gast, S.A. Dzuba, C.R. Timmel, C.E. Fursman, P. Hore, Spectrochim. Spectrochim. Acta Part A Mol. Biomol. Spectrosc. 54(14), 2283 (1998)

206. K. Laukenmann, S. Weber, G. Kothe, C. Oesterle, A. Angerhofer, M.R. Wasielewski, W.A. Svec, J.R. Norris, J. Phys. Chem. 99(12), 4324 (1995)

207. B. Guigliarelli, C. More, A. Fournel, M. Asso, E.C. Hatchikian, R. Williams, R. Cammack, P. Bertrand, Biochemistry 34(14), 4781 (1995)

208. A.W.E. Dilg, F. Capozzi, M. Mentler, O. Iakovleva, C. Luchinat, I. Bertini, F.G. Parak, J. Biol. Inorg. Chem. 6(3), 232 (2001)

209. A.S. Lukas, M.R. Wasielewski, in Molecular Switches, ed. by B.L. Feringa (Wiley-VCH, Weinheim, 2001), pp. 1-35

210. M.R. Wasielewski, Chem. Rev. 92(3), 435 (1992)

211. J.J. Hopfield, J.N. Onuchic, D.N. Beratan, Science 241(4867), 817 (1988)

212. M.P. Debreczeny, W.A. Svec, E.M. Marsh, M.R. Wasielewski, J. Am. Chem. Soc. 118(34), 8174 (1996)

213. E.T. Chernick, Q. Mi, R.F. Kelley, E.A. Weiss, B.A. Jones, T.J. Marks, M.A. Ratner, M.R. Wasielewski, J. Am. Chem. Soc. 128(13), 4356 (2006)

214. V.F. Tarasov, S.S.M. Islam, Y. Ohba, M.D.E. Forbes, S. Yamauchi, Appl. Magn. Reson. 41(2-4), $175(2011)$

215. S. Shaakov, T. Galil, E. Stavitski, H. Levanon, A. Lukas, M.R. Wasielewski, J. Am. Chem. Soc. 125(21), 6563 (2003)

216. A.S. Lukas, P.J. Bushard, E.A. Weiss, M.R. Wasielewski, J. Am. Chem. Soc. 125(13), 3921 (2003)

217. Y. Mori, Y. Sakaguchi, H. Hayashi, J. Phys. Chem. A 104(21), 4896 (2000)

218. Y. Kobori, A. Kawai, K. Obi, J. Phys. Chem. 98(26), 6425 (1994)

219. C.R. Timmel, P.J. Hore, Chem. Phys. Lett. 226(1), 144 (1994)

220. J. Tan, M.C. Thurnauer, J.R. Norris, Chem. Phys. Lett. 219(3), 283 (1994)

221. E.T. Chernick, Q. Mi, A.M. Vega, J.V. Lockard, M.A. Ratner, M.R. Wasielewski, J. Phys. Chem. B 111(24), 6728 (2007)

222. E.M. Giacobbe, Q. Mi, M.T. Colvin, B. Cohen, C. Ramanan, A.M. Scott, S. Yeganeh, T.J. Marks, M.A. Ratner, M.R. Wasielewski, J. Am. Chem. Soc. 131(10), 3700 (2009)

223. R. Carmieli, Q. Mi, A.B. Ricks, E.M. Giacobbe, S.M. Mickley, M.R. Wasielewski, J. Am. Chem. Soc. 131(24), $8372(2009)$

224. Y. Teki, H. Tamekuni, K. Haruta, J. Takeuchi, Y. Miura, J. Mater. Chem. 18, 381 (2008)

225. M.T. Colvin, R. Carmieli, T. Miura, S. Richert, D.M. Gardner, A.L. Smeigh, S.M. Dyar, S.M. Conron, M.A. Ratner, M.R. Wasielewski, J. Phys. Chem. A 117(25), 5314 (2013)

226. D.M. Gardner, H.F. Chen, M.D. Krzyaniak, M.A. Ratner, M.R. Wasielewski, J. Phys. Chem. A 119(29), 8040 (2015)

227. S. Yeganeh, M.R. Wasielewski, M.A. Ratner, J. Am. Chem. Soc. 131(6), 2268 (2009)

228. I. Ciofini, C. Adamo, Y. Teki, F. Tuyèras, P.P. Lainé, Chem. Eur. J. 14(36), 11385 (2008)

229. Y. Teki, T. Matsumoto, Phys. Chem. Chem. Phys. 13, 5728 (2011)

230. T. Matsumoto, Y. Teki, Phys. Chem. Chem. Phys. 14, 10178 (2012) 
231. M.T. Colvin, E.M. Giacobbe, B. Cohen, T. Miura, A.M. Scott, M.R. Wasielewski, J. Phys. Chem. A 114(4), $1741(2010)$

232. I. Matsumoto, I. Ciofini, P.P. Lainé, Y. Teki, Chem. Eur. J. 15(42), 11210 (2009)

233. T. Miura, M.R. Wasielewski, J. Am. Chem. Soc. 133(9), 2844 (2011)

234. M. Sierra, M.A. Herranz, S. Zhang, L. Sánchez, N. Martín, L. Echegoyen, Langmuir 22(25), 10619 (2006)

235. T. Gupta, P.C. Mondal, A. Kumar, Y.L. Jeyachandran, M. Zharnikov, Adv. Funct. Mater. 23(34), 4227 (2013)

236. J.L. Zhang, S. Zhong, J.Q. Zhong, T.C. Niu, W.P. Hu, A.T.S. Wee, W. Chen, Nanoscale 7, 4306 (2015)

237. P. Samorì, X. Yin, N. Tchebotareva, Z. Wang, T. Pakula, F. J. äckel, M. D. Watson, A. Venturini, K. Müllen, J. P. Rabe, J. Am. Chem. Soc. 126(11), 3567 (2004)

238. P.C. Mondal, V. Singh, Y.L. Jeyachandran, M. Zharnikov, ACS Appl. Mater. Interfaces 7(16), 8677 (2015)

239. B.I. Kharisov, O.V. Kharissova, M.J. Gomez, U.O. Mendez, Ind. Eng. Chem. Res. 48(2), 545 (2009)

240. G. Sauvé, R. Fernando, J. Phys. Chem. Lett. 6(18), 3770 (2015)

241. G. Li, R. Zhu, Y. Yang, Nat. Photon. 6, 153 (2012)

242. S. Priyadarshy, M.J. Therien, D.N. Beratan, J. Am. Chem. Soc. 118(6), 1504 (1996)

243. K. Ohkubo, S. Fukuzumi, Bull. Chem. Soc. Jpn. 82(3), 303 (2009)

244. D.M. Guld, M. Maggin, G. Scorrano, M. Prato, J. Am. Chem. Soc. 119(5), 974 (1997)

245. O. Johansson, M. Borgström, R. Lomoth, M. Palmblad, J. Bergquist, L. Hammarström, L. Sun, B. Åkermark, Inorg. Chem. 42(9), 2908 (2003)

246. A. Caneschi, A. Dei, F. Fabrizi de Biani, P. Gütlich, V. Ksenofontov, G. Levchenko, A. Hoefer, F. Renz, Chem. Eur. J. 7(18), 3926 (2001)

247. A. Droghetti, S. Sanvito, Phys. Rev. Lett. 107, 047201 (2011)

248. O. Sato, Proc. Jpn. Acad., Ser. B, Phys. Biol. Sci. 88(6), 213 (2012)

249. P. Gütlich, A. Hauser, H. Spiering, Angew. Chem. Int. Ed. 33(20), 2024 (1994)

250. D.N. Hendrickson, C.G. Pierpont, Valence Tautomeric Transition Metal Complexes, Topics in Current Chemistry, vol. 234 (Springer, Berlin, 2004)

251. O. Sato, A. Cui, R. Matsuda, J. Tao, S. Hayami, Acc. Chem. Res. 40(5), 361 (2007)

252. R.D. Schmidt, D.A. Shultz, J.D. Martin, P.D. Boyle, J. Am. Chem. Soc. 132(17), 6261 (2010)

253. J. Dai, S. Kanegawa, Z. Li, S. Kang, O. Sato, Eur. J. Inorg. Chem. 2013(24), 4150 (2013)

254. A. Hauser, in Spin Crossover in Transition Metal Compounds II. Topics in Current Chemistry, vol. 234, ed. by P. Gütlich, H.A. Goodwin (Springer, Berlin, Heidelberg, 2004) pp. 155-198

255. J.F. Létard, P. Guionneau, L. Goux-Capes, in Spin Crossover in Transition Metal Compounds III. Topics in Current Chemistry, vol. 235 (Springer, Berlin, Heidelberg, 2004), pp. 221-249

256. S. Venkataramani, U. Jana, M. Dommaschk, F.D. Sönnichsen, F. Tuczek, R. Herges, Science 331(6016), 445 (2011)

257. S. Thies, H. Sell, C. Schütt, C. Bornholdt, C. Näther, F. Tuczek, R. Herges, J. Am. Chem. Soc. 133(40), 16243 (2011)

258. M. Milos, S. Kairouani, S. Rabaste, A. Hauser, Coord. Chem. Rev. 252(23-24), 2540 (2008)

259. R. Bertoni, M. Cammarata, M. Lorenc, S.F. Matar, J.F. Létard, H.T. Lemke, E. Collet, Acc. Chem. Res. 48(3), 774 (2015)

260. M.V. Fedin, E.G. Bagryanskaya, H. Matsuoka, S. Yamauchi, S.L. Veber, K.Y. Maryunina, E.V. Tretyakov, V.I. Ovcharenko, R.Z. Sagdeev, J. Am. Chem. Soc. 134(39), 16319 (2012)

261. M.V. Fedin, K.Y. Maryunina, R.Z. Sagdeev, V.I. Ovcharenko, E.G. Bagryanskaya, Inorg. Chem. 51(1), 709 (2012)

262. M. Fedin, V. Ovcharenko, R. Sagdeev, E. Reijerse, W. Lubitz, E. Bagryanskaya, Angew. Chem. Int. Ed. 47(36), 6897 (2008)

263. Y. Mulyana, G. Poneti, B. Moubaraki, K.S. Murray, B.F. Abrahams, L. Sorace, C. Boskovic, Dalton Trans. 39, 4757 (2010)

264. C. Carbonera, A. Dei, J.F. Létard, C. Sangregorio, L. Sorace, Inorg. Chim. Acta 360(13), 3825 (2007)

265. Y.S. Huang, X. Yang, E. Schwartz, L.P. Lu, S. Albert-Seifried, C.E. Finlayson, M. Koepf, H.J. Kitto, B. Ulgut, M.B.J. Otten, J.J.L.M. Cornelissen, R.J.M. Nolte, A.E. Rowan, R.H. Friend, J. Phys. Chem. B 115(7), 1590 (2011)

266. L. Novotny, N. van Hulst, Nat. Photon. 5, 83 (2011) 
267. G. Malheiros-Silveira, L. Gabrielli, C. Chang-Hasnain, H. Hernandez-Figueroa, IEEE Photon. J. 6(2), 1 (2014)

268. L. Tang, S.E. Kocabas, S. Latif, A.K. Okyay, D.S. Ly-Gagnon, K.C. Saraswat, D.A.B. Miller, Nat. Photon. 2, 226 (2008)

269. Y.W. Son, M.L. Cohen, S.G. Louie, Phys. Rev. Lett. 97, 216803 (2006)

270. H. Lee, Y.W. Son, N. Park, S. Han, J. Yu, Phys. Rev. B 72, 174431 (2005)

271. A. Narita, X. Feng, Y. Hernandez, S.A. Jensen, M. Bonn, H. Yang, I.A. Verzhbitskiy, C. Casiraghi, M.R. Hansen, A.H.R. Koch, G. Fytas, O. Ivasenko, B. Li, K.S. Mali, T. Balandina, S. Mahesh, S.D. Feyter, K. Müllen, Nat. Chem. 6, 126 (2014)

272. A. Narita, X.Y. Wang, X. Feng, K. Müllen, Chem. Soc. Rev. 44, 6616 (2015)

273. J. Cai, P. Ruffieux, R. Jaafar, M. Bieri, T. Braun, S. Blankenburg, M. Muoth, A.P. Seitsonen, X.F. Moussa Saleh, K. Müllen, R. Fasel, Nature 466, 470 (2010)

274. M. Slota, M. Blankenhorn, E. Heintze, M. Vu, R. Hübner, L. Bogani, Faraday Discuss. 185, 347 (2015)

275. O. Sato, Nat. Chem. 8, 644 (2016)

276. J.P. Llinas, A. Fairbrother, G.B. Barin, W. Shi, K. Lee, S. Wu, B.Y. Choi, R. Braganza, J. Lear, N. Kau, W. Choi, C. Chen, Z. Pedramrazi, T. Dumslaff, A. Narita, X. Feng, K. Müllen, F. Fischer, A. Zettl, P. Ruffieux, E. Yablonovitch, M. Crommie, R. Fasel, J. Bokor, Nat. Commun. 8(1), 633 (2017)

277. M. Slota, A. Keerthi, W.K. Myers, E. Tretyakov, M. Baumgarten, A. Ardavan, H. Sadeghi, C.J. Lambert, A. Narita, K. Müllen, L. Bogani, Nature 557(7707), 691 (2018)

278. K. Nakada, M. Fujita, G. Dresselhaus, M.S. Dresselhaus, Phys. Rev. B 54, 17954 (1996)

279. M. Fujita, K. Wakabayashi, K. Nakada, K. Kusakabe, J. Phys. Soc. Jpn. 65(7), 1920 (1996)

280. A. Saffarzadeh, R. Farghadan, Appl. Phys. Lett. 98(2), 023106 (2011)

281. R. Farghadan, A. Saffarzadeh, RSC Adv. 5, 87411 (2015)

282. F. Lombardi, A. Lodi, J. Ma, J. Liu, M. Slota, A. Narita, W.K. Myers, K. Müllen, X. Feng, L. Bogani, Science 366(6469), 1107 (2019)

283. F. Lombardi, W.K. Myers, J. Ma, J. Liu, X. Feng, L. Bogani, Phys. Rev. B 101, 094406 (2020)

284. K. Yang, W. Paul, S.H. Phark, P. Willke, Y. Bae, T. Choi, T. Esat, A. Ardavan, A.J. Heinrich, C.P. Lutz, Science 366(6464), 509 (2019)

Publisher's Note Springer Nature remains neutral with regard to jurisdictional claims in published maps and institutional affiliations. 